\title{
Primitive bound of a 2-structure
}

\author{
Abderrahim Boussaïri, Pierre Ille, and Robert E. Woodrow
}

\begin{abstract}
A 2-structure on a set $S$ is given by an equivalence relation on the set of ordered pairs of distinct elements of $S$. A subset $C$ of $S$, any two elements of which appear the same from the perspective of each element of the complement of $C$, is called a clan. The number of elements that must be added in order to obtain a 2structure the only clans of which are trivial is called the primitive bound of the 2-structure. The primitive bound is determined for arbitrary 2-structures of any cardinality. This generalizes the classical results of Erdős et al. and Moon for tournaments, as well as the result of Brignall et al. for finite graphs, and the precise results of Boussaïri and Ille for finite graphs, providing new proofs which avoid extensive use of induction in the finite case.
\end{abstract}

MSC 2010 SUBJECt Classifications: Primary 05C70, 05C69, 05C63. KEYWORDS AND PHRASES: 2-structure, clan, primitive 2-structure, primitive extension, primitive bound, clan completeness.

\section{Introduction ${ }^{1}$}

The notion of a primitive [10, 13] (also called indecomposable [14, 20], prime $[8,17]$ or simple $[3,4,11,12,15,24])$ structure has been studied in the context of graphs, tournaments, more general structures derived from binary relations, and in general for relational structures by Fraïssé [13, 14]. Key is the idea of a subset the elements of which look the same from the perspective of each element of the complement, called autonomous set [16, 22, 23], clan [10], convex set [11, 12], homogeneous set [8], interval [14, 20, 25], module $[1,17,26]$ or partitive set [28]. An indecomposable structure is one for which all such subsets are trivial.

Given a structure, it is natural to ask about embedding it into an indecomposable structure and to seek to mimimize the number of elements one must add. In the early 70's this was done by Sumner [27] for finite complete graphs, by Moon [24] for finite tournaments and by Erdős et al. [12] for arbitrary tournaments. More recently the question was revived by Brignall [3] and Brignall et al. [4] for finite graphs and other finite combinatorial

\footnotetext{
${ }^{1} \mathrm{~A}$ symbol glossary is provided at the end of the paper.
} 
structures. Boussairi and Ille [2] provided a detailed analysis and identified the precise parameter to describe the situation for finite graphs. In this work we yield a unified approach by studying the more general situation of 2-structures [10]. The techniques introduced permit us to generalize the parameter obtained by Boussairi and Ille [2] to the arbitrary setting.

Resolution of the problem for tournaments by Erdős et al. [11] employed a linearization of the tournament and the Bernstein Property. We introduce the notion of a traverse of an arbitrary 2-structure which respects key clans of the 2-structure and the notion of a dense bicoloring mirroring the Bernstein Property. We also introduce the notion of inclusive clans and develop their structural properties. These tools permit us to provide precise bounds and to present a new proof of the now classical result of Erdös et al. [12] that adds structural understanding and does not turn on induction to handle the finite case.

The notion of traverse plays an important role in the algorithmics for the finite graphs as well. There a traverse induces a permutation of the vertex set called a factorizing permutation [5]. Factorizing permutations are used to find efficient algorithms which compute the clan tree. Given a finite graph, the first step consists in calculating a factorizing permutation [18] and the second in determining the clan tree from a factorizing permutation [6].

At present, we formalize our presentation. A 2-structure $\sigma$ consists of an infinite or finite vertex set (or domain [10]) $V(\sigma)$ and of an equivalence relation $\equiv_{\sigma}$ defined on $(V(\sigma) \times V(\sigma)) \backslash\{(v, v): v \in V(\sigma)\}$. An equivalence class of $\equiv_{\sigma}$ is also called a class of $\sigma$. The family of the classes of $\sigma$ is denoted by $E(\sigma)$. Given a 2-structure $\sigma$, consider $W \subseteq V(\sigma)$. The 2-substructure $\sigma[W]$ of $\sigma$ induced by $W$ is the 2-structure defined on $V(\sigma[W])=W$ such that $\equiv_{\sigma[W]}$ coincides with the restriction of $\equiv_{\sigma}$ to $(W \times W) \backslash\{(w, w): w \in$ $W\}$. Given $W \subseteq V(\sigma), \sigma[V(\sigma) \backslash W]$ is denoted by $\sigma-W$, and by $\sigma-w$ when $W=\{w\}$.

With each 2-structure $\sigma$ associate the 2-structure $\sigma^{\star}$ defined on the same vertex set by

$$
(u, v) \equiv_{\sigma^{\star}}(x, y) \quad \text { if } \quad(v, u) \equiv_{\sigma}(y, x)
$$

for distinct $u, v \in V\left(\sigma^{\star}\right)$ and distinct $x, y \in V\left(\sigma^{\star}\right)$. A 2-structure $\sigma$ is reversible if $\sigma=\sigma^{\star}$ and non-reversible otherwise. Let $\sigma$ be a reversible 2structure. For each $e \in E(\sigma), e^{\star}=\{(u, v):(v, u) \in e\} \in E(\sigma)$ and we have either $e=e^{\star}$ or $e \cap e^{\star}=\emptyset$. In the first instance, $e$ is said to be symmetric. It is called asymmetric in the second. A reversible 2-structure $\sigma$ is symmetric when all the classes of $\sigma$ are symmetric, it is asymmetric when all its classes are asymmetric. 
A graph $\Gamma=(V(\Gamma), E(\Gamma))$ is identified with the symmetric 2-structure $\sigma(\Gamma)$ defined on $V(\sigma(\Gamma))=V(\Gamma)$ as follows. For distinct $u, v \in V(\Gamma)$ and distinct $x, y \in V(\Gamma),(u, v) \equiv_{\sigma(\Gamma)}(x, y)$ if either $\{u, v\},\{x, y\} \in E(\Gamma)$ or $\{u, v\},\{x, y\} \notin E(\Gamma)$. Notice that $\sigma(\Gamma)=\sigma(\bar{\Gamma})$, where $\bar{\Gamma}=\left(V(\Gamma),\left(\begin{array}{c}V(\Gamma) \\ 2\end{array}\right) \backslash\right.$ $E(\Gamma))$ is the complement of $\Gamma$. Similarly, a tournament $T=(V(T), A(T))$ is identified with the asymmetric 2-structure $\sigma(T)$ defined on $V(\sigma(T))=V(T)$ as follows. For distinct $u, v \in V(T)$ and distinct $x, y \in V(T),(u, v) \equiv_{\sigma(T)}$ $(x, y)$ if either $(u, v),(x, y) \in A(T)$ or $(u, v),(x, y) \notin A(T)$. Notice that $\sigma(T)=\sigma\left(T^{\star}\right)$, where $T^{\star}=\left(V(T), A(T)^{\star}\right)$ is the dual of $T$.

Let $\sigma$ be a 2-structure. A subset $C$ of $V(\sigma)$ is a clan [10] of $\sigma$ if for any $c, d \in C$ and $v \in V(\sigma) \backslash C$, we have

$$
(c, v) \equiv_{\sigma}(d, v) \text { and }(v, c) \equiv_{\sigma}(v, d) .
$$

For instance, $\emptyset, V(\sigma)$ and $\{v\}$, for $v \in V(\sigma)$, are clans of $\sigma$ called trivial clans of $\sigma$. A 2-structure is indecomposable if all its clans are trivial. A 2-structure $\sigma$ is primitive [10] if $\sigma$ is indecomposable with $|V(\sigma)| \geq 3$. Otherwise $\sigma$ is said to be imprimitive [10].

Given a set $S$ with $|S| \geq 2$, Sumner [27, Theorem 2.45] observed that the complete graph $K_{S}$ admits a primitive graph extension $G$ such that $|V(G) \backslash S|=\left\lceil\log _{2}(|S|+1)\right\rceil$. This is extended to any graph in [3, Theorem 3.7] and [4, Theorem 3.2] as follows. A graph $G$, with $|V(G)| \geq 2$, admits a primitive graph extension $H$ such that $|V(H) \backslash V(G)|=\left\lceil\log _{2}(|V(G)|+1)\right\rceil$.

Definition 1.1. A 2-structure $\tau$ is an extension of a 2-structure $\sigma$ if $V(\tau) \supseteq$ $V(\sigma)$ and $\tau[V(\sigma)]=\sigma$. Given a cardinal $\kappa$, a $\kappa$-extension of a 2-structure $\sigma$ is an extension $\tau$ of $\sigma$ such that $|V(\tau) \backslash V(\sigma)|=\kappa$. Let $\tau$ be an extension of $\sigma$. Consider the function $\sigma \hookrightarrow \tau: E(\sigma) \longrightarrow E(\tau)$ satisfying $(\sigma \hookrightarrow \tau)(e) \supseteq e$ for every $e \in E(\sigma)$. Clearly $\sigma \hookrightarrow \tau$ is injective and we can identify $(\sigma \hookrightarrow \tau)(e)$ with $e$ for every $e \in E(\sigma)$. We say that $\tau$ is a faithful extension of $\sigma$ if

$$
\sigma \hookrightarrow \tau \text { is bijective }
$$

and for any $e, f \in E(\sigma)$,

$$
(\sigma \hookrightarrow \tau)(e) \cap((\sigma \hookrightarrow \tau)(f))^{\star} \neq \emptyset \Longrightarrow e \cap f^{\star} \neq \emptyset .
$$

The necessity of Conditions (1.1) and (1.2) is discussed in Section 8. In Sumner's result, the primitive graph extension is not a faithful extension of the complete graph. A 2-structure $\sigma$ is complete [10] if $|V(\sigma)| \leq 1$ or $|V(\sigma)| \geq 2$ and $|E(\sigma)|=1$. Let $\sigma$ be a 2-structure. Clearly, the subsets 
$W$ of $V(\sigma)$ such that $\sigma[W]$ is complete correspond to cliques and stable sets in a graph. As already observed in Sumner's result, to obtain primitive extensions of a complete 2-structure, we consider symmetric 2-structures $\sigma$ with $|E(\sigma)|=2$.

Definition and notation 1.2. Let $\sigma$ be a 2 -structure admitting a primitive and faithful extension. The primitive bound $p(\sigma)$ of $\sigma$ is the smallest cardinal $\kappa$ such that $\sigma$ possesses a primitive and faithful $\kappa$-extension.

Brignall et al. [4] obtained that

$$
p(G) \leq\left\lceil\log _{2}(|V(G)|+1)\right\rceil
$$

for any finite graph $G$. In his Ph.D. Thesis, Brignall [3] conjectured that

$$
p(G) \leq\left\lceil\log _{2}(\max (\alpha(G), \omega(G))+1)\right\rceil .
$$

Boussaïri and Ille [2] identified the correct parameter

$$
c(G)=\max (\{|C|: C \text { is a clan of } G \text { which is a stable set or a clique }\})
$$

and proved for every finite graph $G$ that

$$
\left\lceil\log _{2}(c(G))\right\rceil \leq p(G) \leq\left\lceil\log _{2}(c(G)+1)\right\rceil .
$$

Thus $p(G)=\left\lceil\log _{2}(c(G))\right\rceil$ when $\log _{2}(c(G)) \notin \mathbb{N}$. When $c(G)=2^{k}$ with $k \geq 1$, we have $p(G)=k$ or $k+1$, and Boussaïri and Ille [2] showed that $p(G)=k+1$ if and only if $G$ (or $\bar{G}$ ) admits $2^{k}$ isolated vertices.

Definition 1.3. Consider a reversible 2-structure $\sigma$. A vertex $v$ of $\sigma$ is isolated if there exists a symmetric class $e$ of $\sigma$ such that $(v, w) \in e$ for every $w \in V(\sigma) \backslash\{v\}$. Note that if $v_{1}$ and $v_{2}$ are distinct isolated vertices, then the classes $e_{1}$ and $e_{2}$ in the definition are equal.

Definition and notation 1.4. For a 2-structure $\sigma$, we introduce the clan completness $c(\sigma)$ of $\sigma$ as being the supremum of cardinalities of clans $C$ of $\sigma$ such that $\sigma[C]$ is complete.

The paper is organized as follows. In Section 2, we recall the definition of the clan tree of a 2-structure, and we state some of its properties (see Lemmas 2.9, 2.10 and 2.11). In Section 3, we introduce the tree equivalence associated with a 2-structure (see Definition and notation 3.5), and we characterize its nontrivial equivalence classes (see Theorem 3.7). In Section 4, we 
introduce the notion of a traverse (see Definition 4.1). We establish the existence of traverses for each 2-structure (see Proposition 4.2), and we study the intervals of traverses (see Lemma 4.7, and Corollaries 4.8 and 4.9). In section 5, we describe two ways of constructing faithful extensions (see Lemmas 5.3 and 5.4). We also examine the clans of specific faithful extensions (see Lemma 5.7 and Corollary 5.8). In Section 6, we introduce the notion of an inclusive clan, and we provide interesting properties of inclusive clans (for instance, see Propositions 6.5 and 6.6, and Theorem 6.7).

In Section 7, we determine the primitive bounds of reversible 2-structures. More precisely, we proceed in the following manner. For an infinite or finite tournament $T$, Erdős et al. [11] established that $p(T) \leq 2$. Then Moon [24] proved for a finite tournament $T$ such that $|V(T)| \geq 4$ that $p(T)=2$ if and only if $T$ is an odd linear order. Erdös et al. [12] extended this result to tournaments of arbitrary cardinality. Using dense bicolorings of traverses (see Section 4) and inclusive clans (see Section 6), we provide an elegant proof of [12] (see Theorem 7.1).

Given a reversible 2-structure $\sigma$ such that $2 \leq|E(\sigma)| \leq c(\sigma)<\aleph_{0}$, we prove in Corollary 7.5 that

$$
\left\lceil\log _{|E(\sigma)|}(c(\sigma))\right\rceil \leq p(\sigma) \leq\left\lceil\log _{|E(\sigma)|}(c(\sigma)+1)\right\rceil .
$$

Moreover, when $c(\sigma)=|E(\sigma)|^{k}$ where $k \geq 1$, we show in Theorem 7.8 that $p(\sigma)=k+1$ if and only if $\sigma$ admits exactly $|E(\sigma)|^{k}$ isolated vertices.

The cardinal logarithm is defined as follows. Given cardinals $\mu$ and $\nu$,

$$
\log _{\mu}(\nu)=\min \left(\left\{\kappa: \mu^{\kappa} \geq \nu\right\}\right) .
$$

If $\mu$ and $\nu$ are finite, then $\log _{\mu}(\nu)=\left\lceil\log _{\mu}(\nu)\right\rceil$. Given a reversible 2-structure $\sigma$ such that $|E(\sigma)| \geq 2$, we establish in Theorem 7.9 that

$$
\mathfrak{l o g}_{|E(\sigma)|}(c(\sigma)) \geq \aleph_{0} \Longrightarrow p(\sigma)=\mathfrak{l o g}_{|E(\sigma)|}(c(\sigma)) .
$$

We determine the primitive bounds of the other reversible 2-structures in Theorem 7.2. In particular, we obtain the following result for a reversible 2 -structure $\sigma$ such that $c(\sigma)=1$. If $\sigma$ has at least three classes or if $\sigma$ is symmetric and has two classes, then $p(\sigma) \leq 1$.

In Section 8, we determine the primitive bounds of non-reversible 2structures. We proceed as follows. With 2-structures $\sigma$ and $\tau$ such that 
$V(\sigma)=V(\tau)$ associate the 2-structure $\sigma \wedge \tau$ defined on the same vertex set by

$$
E(\sigma \wedge \tau)=\{e \cap f: e \in E(\sigma), f \in E(\tau) \text { and } e \cap f \neq \emptyset\} .
$$

For an arbitrary 2-structure $\sigma, \sigma \wedge \sigma^{\star}$ is reversible, and we prove in Theorem 8.5 that $p(\sigma)=p\left(\sigma \wedge \sigma^{\star}\right)$.

\section{Clan tree}

Notation 2.1. Let $\sigma$ be a 2-structure. For distinct $u, v \in V(\sigma)$, the class of $\sigma$ containing $(u, v)$ is denoted by $(u, v)_{\sigma}$. For $W, W^{\prime} \subseteq V(\sigma)$, with $W \cap W^{\prime}=\emptyset$, $W \longleftrightarrow W^{\prime}$ signifies that $\left(v, v^{\prime}\right)_{\sigma}=\left(w, w^{\prime}\right)_{\sigma}$ and $\left(v^{\prime}, v\right)_{\sigma}=\left(w^{\prime}, w\right)_{\sigma}$ for any $v, w \in W$ and $v^{\prime}, w^{\prime} \in W^{\prime}$. Given $v \in V(\sigma)$ and $W \subseteq V(\sigma) \backslash\{v\},\{v\} \longleftrightarrow{ }_{\sigma} W$ is also denoted by $v \longleftrightarrow{ }_{\sigma} W$. The negation is denoted by $v \nLeftarrow \longleftrightarrow_{\sigma} W$. Let $W, W^{\prime} \subseteq V(\sigma)$ such that $W \cap W^{\prime}=\emptyset$ and $W \longleftrightarrow W^{\prime}$. The class of $\sigma$ containing $\left(w, w^{\prime}\right)$, where $w \in W$ and $w^{\prime} \in W^{\prime}$, is denoted by $\left(W, W^{\prime}\right)_{\sigma}$. Given $W \subsetneq V(\sigma)$ and $v \in V(\sigma) \backslash W$ such that $v \longleftrightarrow \sigma W,(\{v\}, W)_{\sigma}$ is also denoted by $(v, W)_{\sigma}$.

Let $\sigma$ be a 2-structure. Using Notation 2.1, a subset $C$ of $V(\sigma)$ is a clan of $\sigma$ if and only if for each $v \in V(\sigma) \backslash C$, we have $v \longleftrightarrow{ }_{\sigma} C$.

Notation 2.2. The set of clans of a 2-structure $\sigma$ is denoted by $\mathcal{C}$ lans $(\sigma)$.

Given a 2-structure $\sigma$, a partition $\mathbb{F}$ of $V(\sigma)$ is a factorization [10] of $\sigma$ if all the elements of $\mathbb{F}$ are clans of $\sigma$. Let $\mathbb{F}$ be a factorization of $\sigma$. Given distinct $X, Y \in \mathbb{F}$, we have $X \longleftrightarrow_{\sigma} Y$ because $X \cap Y=\emptyset$. Thus there is $e \in E(\sigma)$ such that $(X, Y)_{\sigma}=e$. This justifies the following definition. The quotient of $\sigma$ by $\mathbb{F}$ is the 2-structure $\sigma / \mathbb{F}$ defined on $V(\sigma / \mathbb{F})=\mathbb{F}$ as follows. For distinct $X, Y \in \mathbb{F}$ and distinct $X^{\prime}, Y^{\prime} \in \mathbb{F}$,

$$
(X, Y) \equiv_{\sigma / \mathbb{F}}\left(X^{\prime}, Y^{\prime}\right) \text { if }(X, Y)_{\sigma}=\left(X^{\prime}, Y^{\prime}\right)_{\sigma}
$$

Hence, for distinct $X, Y \in \mathbb{F}$ and distinct $X^{\prime}, Y^{\prime} \in \mathbb{F},(X, Y) \equiv_{\sigma / \mathbb{F}}\left(X^{\prime}, Y^{\prime}\right)$ if and only if $(x, y) \equiv_{\sigma}\left(x^{\prime}, y^{\prime}\right)$, where $x \in X, y \in Y, x^{\prime} \in X^{\prime}$ and $y^{\prime} \in Y^{\prime}$.

The following strengthening of the notion of clan is useful to present the clan decomposition theorem. Given a 2-structure $\sigma$, a clan $C$ of $\sigma$ is said to be strong provided that for every clan $D$ of $\sigma$, we have:

$$
\text { if } C \cap D \neq \emptyset \text {, then } C \subseteq D \text { or } D \subseteq C \text {. }
$$

Notation 2.3. The set of strong clans of a 2-structure $\sigma$ is denoted by $\mathcal{S} \operatorname{trong}(\sigma)$. 
Notation 2.4. We associate with a 2-structure $\sigma$ the Gallai family $\mathcal{G}$ allai $(\sigma)$ of the maximal elements under inclusion of $\mathcal{S} \operatorname{trong}(\sigma) \backslash\{\emptyset, V(\sigma)\}$.

The clan decomposition theorem is stated as follows. It is attributable to Gallai $[16,23]$ for finite graphs (see [10, Theorem 5.5] for finite 2-structures, and [19, Theorem 4.2] for infinite ones).

Recall that an asymmetric 2-structure $\sigma$, with $|V(\sigma)| \geq 2$, is linear [10] if there is $e \in E(\sigma)$ such that $(V(\sigma), e)$ is a linear order.

Theorem 2.5. For a 2-structure $\sigma$ such that $\mathcal{G}$ allai $(\sigma) \neq \emptyset$, the family $\mathcal{G}$ allai $(\sigma)$ realizes a factorization of $\sigma$. Moreover, the corresponding quotient $\sigma / \mathcal{G}$ allai $(\sigma)$ is complete, linear or primitive.

Notation 2.6. Given a 2-structure $\sigma$, consider a subset $W$ of $V(\sigma)$ such that $\mathcal{G}$ allai $(\sigma[W]) \neq \emptyset$. The quotient $\sigma[W] / \mathcal{G}$ allai $(\sigma[W])$ is denoted by

$$
\mathcal{Q u o t i e n t}_{\sigma}(W) \text {. }
$$

Let $\sigma$ be a 2-structure. Given a strong clan $C$ of $\sigma$, note that all the strong clans of $\sigma[C]$ are strong clans of $\sigma$. A strong clan $C$ of $\sigma$ is a limit of $\sigma$ if $\mathcal{G}$ allai $(\sigma[C])=\emptyset$.

Notation 2.7. Let $\sigma$ be a 2-structure. We associate with $\sigma$ the subset $\mathcal{T}$ ree $(\sigma)$ of $\mathcal{S}$ trong $(\sigma)$ defined as follows. Given a strong clan $C$ of $\sigma, C \in$ $\mathcal{T}$ ree $(\sigma)$ if one of the next two assertions holds

- $C$ is not a limit of $\sigma$;

- there exists a strong clan $D$ of $\sigma$, which is not a limit of $\sigma$, such that $C \in \mathcal{G}$ allai $(\sigma[D])$.

Sometimes, the singletons are added to $\mathcal{T} \operatorname{ree}(\sigma)$ when $V(\sigma)$ is infinite. As a direct consequence of the definition of a strong clan, we obtain that the family $\mathcal{T}$ ree $(\sigma)$, endowed with inclusion, is a tree called the clan tree of $\sigma$. For clan trees of finite digraphs, see [7]. For infinite digraphs, see [9]. Lastly, for infinite 2-structures or more generally for weakly partitive families on infinite sets, see [21]. When $V(\sigma)$ is finite, we have

$$
\mathcal{T} \text { ree }(\sigma)=\mathcal{S} \operatorname{trong}(\sigma) \backslash\{\emptyset\} .
$$

Notation 2.8. Let $\sigma$ be a 2-structure. Given a subset $W$ of $V(\sigma)$ such that $W \neq \emptyset$, the intersection of all the strong clans $C$ of $\sigma$ such that $C \supseteq W$ is a strong clan of $\sigma$. It is denoted by $\widetilde{W}$. Similarly, given a subset $W$ of $V(\sigma)$ such that $W \neq \emptyset$ and $W \neq V(\sigma)$, the intersection of all the strong clans $C$ of $\sigma$ such that $C \supsetneq W$ is a strong clan of $\sigma$. It is denoted by $\widehat{W}$. 
Since the proofs of the next three lemmas are easy, we omit them. The first two are closely related to similar results in [21] (see [21, Lemma 4.1] for the first one, and see [21, Theorem 3.9] for the second one).

Lemma 2.9. Let $\sigma$ be a 2-structure. For distinct $v, w \in V(\sigma), \widetilde{\{v, w\}}$ is a strong clan of $\sigma$ which is not a limit of $\sigma$, and there exist $X_{v}, X_{w} \in$ $\mathcal{G}$ allai $\left(\sigma[\widetilde{\{v, w\}]})\right.$ such that $X_{v} \neq X_{w}, v \in X_{v}$ and $w \in X_{w}$.

Lemma 2.10. Let $\sigma$ be a 2-structure. For $C \subseteq V(\sigma), C$ is a clan of $\sigma$ which is not a strong clan of $\sigma$ if and only if $\widetilde{C}$ is not a limit of $\sigma$, and there exists a nontrivial clan $\mathscr{C}$ of $\mathcal{Q}$ uotient $_{\sigma}(\widetilde{C})$ such that $C=\bigcup_{X \in \mathscr{C}} X$.

Lemma 2.11. Given a 2-structure $\sigma$, consider a strong clan $C$ of $\sigma$ such that $C \neq \emptyset$.

1. $C=\widehat{C}$ if and only if for each $X \in \mathcal{S} \operatorname{trong}(\sigma)$, with $X \supsetneq C$, and for every $x \in X \backslash C$, there is a strong clan $Z$ of $\sigma$, which is not a limit of $\sigma$, such that $C \subsetneq Z \subseteq X \backslash\{x\}$.

2. $C \subsetneq \widehat{C}$ if and only if $\widehat{C}$ is not a limit of $\sigma$, and $C \in \mathcal{Q u o t i e n t}_{\sigma}(\widehat{C})$.

\section{Clan completness and tree equivalence}

Let $\sigma$ be an imprimitive and reversible 2-structure. Suppose that $\sigma$ is finite. To build a primitive extension of $\sigma$, we have to destroy all the nontrivial clans of $\sigma$ by external elements. In fact, it is sufficient to destroy the minimal nontrivial clans of $\sigma$. It follows from Theorem 2.5 that if $C$ is a minimal nontrivial clan of $\sigma$, then $\sigma[C]$ is complete, linear or primitive. If $\sigma[C]$ is complete or linear, then it follows from the minimality of $C$ that $|C|=2$. This leads us to consider the following three families.

Notation 3.1. Let $\sigma$ be a 2 -structure.

- Complete $(\sigma)$ denotes the family of the maximal clans of $\sigma$ under inclusion among the clans $C$ of $\sigma$ such that $|C| \geq 2$ and $\sigma[C]$ is complete;

- $\mathcal{L}$ inear $(\sigma)$ denotes the family of the maximal clans of $\sigma$ under inclusion among the clans $L$ of $\sigma$ such that $|L| \geq 2$ and $\sigma[L]$ is linear;

- Primitive $(\sigma)$ denotes the family of the clans $P$ of $\sigma$ such that $\sigma[P]$ is primitive.

When $\mathcal{C}$ omplete $(\sigma) \neq \emptyset$, the clan completness $c(\sigma)$ of $\sigma$ satisfies

$$
c(\sigma)=\sup (\{|C|: C \in \mathcal{C} \text { omplete }(\sigma)\}) .
$$


Let $\sigma$ be a 2-structure. We characterize the elements of $\mathcal{C}$ omplete $(\sigma) \cup$ $\mathcal{L}$ inear $(\sigma) \cup \mathcal{P}$ rimitive $(\sigma)$. This characterization leads us to introduce from the clan tree of $\sigma$ an equivalence relation, the nontrivial equivalence classes of which are exactly the elements of $\mathcal{C}$ omplete $(\sigma) \cup \mathcal{L}$ inear $(\sigma) \cup \mathcal{P}$ rimitive $(\sigma)$. We omit the proofs because they are somewhat technical, sometimes long and they do not present a major interest in our topics.

Lemma 3.2. Given a 2-structure $\sigma$, consider a clan $C$ of $\sigma$ such that $|C| \geq$ 2 .

1. If $\sigma[C]$ is complete, then there exists $C^{\prime} \in \mathcal{C}$ omplete $(\sigma)$ such that $C^{\prime} \supseteq$ $C$.

2. If $\sigma[C]$ is linear, then there exists $L \in \mathcal{L}$ inear $(\sigma)$ such that $L \supseteq C$.

We characterize the elements of $\mathcal{C}$ omplete $(\sigma) \cup \mathcal{L}$ inear $(\sigma) \cup \mathcal{P} \operatorname{rimitive}(\sigma)$ in terms of the clan tree of $\sigma$.

Proposition 3.3. For a 2-structure $\sigma$, the following three equivalences hold.

1. Given $C \subseteq V(\sigma)$ with $|C| \geq 2, C \in \mathcal{C}$ omplete $(\sigma)$ if and only if $\widetilde{C}$ is a strong clan of $\sigma$, which is not a limit of $\sigma$, such that

- $\operatorname{Quotient}_{\sigma}(\widetilde{C})$ is complete;

- $C=\{c \in \widetilde{C}:\{c\} \in \mathcal{G}$ allai $(\sigma[\widetilde{C}])\}$.

2. Given $L \subseteq V(\sigma)$ with $|L| \geq 2, L \in \mathcal{L}$ inear $(\sigma)$ if and only if $\widetilde{L}$ is a strong clan of $\sigma$, which is not a limit of $\sigma$, such that

- $\operatorname{Quotient}_{\sigma}(\widetilde{L})$ is linear;

- for each $l \in L,\{l\} \in \mathcal{G}$ allai $(\sigma[\tilde{L}])$;

- L is a maximal clan of $\sigma(\widetilde{L})$ under inclusion among the clans $M$ of $\sigma(\widetilde{L})$ such that $\{m\} \in \mathcal{G}$ allai $(\sigma[\widetilde{L}])$ for every $m \in M$.

3. Given $P \subseteq V(\sigma), P \in \mathcal{P}$ rimitive $(\sigma)$ if and only if $P$ is a strong clan of $\sigma$, which is not a limit of $\sigma$, such that

- Quotient $_{\sigma}(P)$ is primitive;

- for every $X \in \mathcal{G}$ allai $(\sigma[\widetilde{L}]),|X|=1$.

The next result follows from Lemma 3.2 and Proposition 3.3.

Corollary 3.4. Let $\sigma$ be a 2-structure. Given distinct $v, w \in V(\sigma)$, the following two assertions are equivalent

- there exists $X \in \mathcal{C}$ omplete $(\sigma) \cup \mathcal{L}$ inear $(\sigma) \cup \mathcal{P}$ rimitive $(\sigma)$ such that $v, w \in X$ 
- $\widehat{\{v\}}=\widehat{\{w\}}$ and there exists a clan $F$ of $\mathcal{Q u o t i e n t}_{\sigma}(\widehat{\{v\}})$ such that $\{v\},\{w\} \in F$ and $F \subseteq\{X \in \mathcal{G}$ allai $(\sigma[\widehat{\{v\}]}):|X|=1\}$.

Corollary 3.4 leads us to introduce the following equivalence relation.

Definition and notation 3.5. Let $\sigma$ be a 2-structure. Given distinct $v, w \in$ $V(\sigma), v \simeq_{\sigma} w$ if $\widehat{\{v\}}=\widehat{\{w\}}$ and if there exists a clan $F$ of Quotient $_{\sigma}(\widehat{\{v\}})$ such that $\{v\},\{w\} \in F$ and $F \subseteq\{X \in \mathcal{G}$ allai $(\sigma[\widetilde{[v\}]}):|X|=1\}$. The equivalence relation $\simeq_{\sigma}$ is called the tree equivalence of $\sigma$.

Notation 3.6. Given a 2-structure $\sigma$, the set of the equivalence classes of $\simeq_{\sigma}$ is denoted by $V(\sigma) / \simeq_{\sigma}$.

The next result is an immediate consequence of Corollary 3.4.

Theorem 3.7. For a 2-structure $\sigma$,

$$
\left\{D \in\left(V(\sigma) / \simeq_{\sigma}\right):|D| \geq 2\right\}=\mathcal{C} \text { omplete }(\sigma) \cup \mathcal{L} \operatorname{inear}(\sigma) \cup \mathcal{P} \operatorname{rimitive}(\sigma) .
$$

Notation 3.8. Given a 2-structure $\sigma$, set

$$
V_{1}(\sigma)=\left\{v \in V(\sigma):\{v\} \in\left(V(\sigma) / \simeq_{\sigma}\right)\right\},
$$

and consider

$$
V_{\downarrow}(\sigma)=\left\{v \in V_{1}(\sigma): \widehat{\{v\}}=\{v\}\right\} .
$$

Remark 3.9. There exist reversible 2-structures $\sigma$ such that $V(\sigma)=V_{\downarrow}(\sigma)$. Precisely, we can construct graphs, that is, symmetric 2-structures $\sigma$ such that $|E(\sigma)|=2$ and $V(\sigma)=V_{\downarrow}(\sigma)$. The existence of such 2-structures constitutes the main difference with the finite case (see Boussairi and Ille [2]). For such 2-structures $\sigma$, it follows from Theorem 3.7 that $\mathcal{C}$ omplete $(\sigma)=\emptyset$. Hence $c(\sigma)=1$. By Theorem 7.2, we have $p(\sigma)=1$. The proof of Theorem 7.2 uses properties of traverses and inclusive clans introduced in Sections 4 and 6 .

\section{Traverses of a 2-structure}

Let $\sigma$ be a reversible 2 -structure such that $\mathcal{L}$ inear $(\sigma) \neq \emptyset$. Recall that $\sigma[L]$ is linear for every $L \in \mathcal{L}$ inear $(\sigma)$. Let $L \in \mathcal{L}$ inear $(\sigma)$. To build a primitive extension of $\sigma$, we have to destroy all the nontrivial clans of $\sigma[L]$ by external elements. Using the Axiom of Choice, we can associate with each $L \in \mathcal{L}$ inear $(\sigma)$ a linear order $\lambda_{L}$ defined on $L$ such that the arc set of $\lambda_{L}$ is one of the two classes of $\sigma[L]$. Let $L \in \mathcal{L}$ inear $(\sigma)$. Clearly, the clans of 
$\sigma[L]$ are exactly the intervals of $\lambda_{L}$. Hence, to build a primitive extension of $\sigma$, we have to destroy all the nontrivial intervals of $\lambda_{L}$, which is possible by using one or two external elements (see Corollary 4.14). This leads us to look for a linear order defined on $V(\sigma)$ which is a common extension of $\lambda_{L}$ when $L \in \mathcal{L}$ inear $(\sigma)$. Therefore, we introduce the following notion.

Definition 4.1. Given a 2-structure $\sigma$, a linear order $L$ defined on $V(\sigma)$ is a traverse of $\sigma$ if the following two assertions hold.

(A1) For each strong clan $X$ of $\sigma, X$ is an interval of $L$.

(A2) Let $X$ be a strong clan of $\sigma$, which is not a limit of $\sigma$, such that

$\mathcal{Q u o t i e n t}_{\sigma}(X)$ is linear.

By Assertion A1, $\mathcal{G}$ allai $(\sigma[X])$ is a factorization of $L[X]$. We require that the arc set of the linear order $L[X] / \mathcal{G}$ allai $(\sigma[X])$ is one of the two classes of $\mathcal{Q u o t i e n t}_{\sigma}(X)$.

Proposition 4.2 (Axiom of Choice). Any 2-structure admits a traverse.

Proof. Consider a 2-structure $\sigma$. Using the Axiom of Choice, we associate with each strong clan $X$ of $\sigma$, which is not a limit of $\sigma$, a linear order $L_{X}$ defined on $\mathcal{G}$ allai $(\sigma[X])$. Moreover, whenever $\mathcal{Q u t i e n t}_{\sigma}(X)$ is linear, we require that the arc set of $L_{X}$ is one of the two classes of $\mathcal{Q}$ uotient $\sigma(X)$.

Now consider the digraph $T$ defined on $V(\sigma)$ as follows. Consider distinct $v, w \in V(\sigma)$. By Lemma 2.9, $\widetilde{\{v, w\}}$ is a strong clan of $\sigma$, which is not a limit of $\sigma$, and there exist distinct $X_{v}, X_{w} \in \mathcal{G}$ allai $\left(\sigma[\widetilde{\{v, w\}})\right.$ such that $v \in X_{v}$ and $w \in X_{w}$. Set

$$
(v, w) \in A(T) \quad \text { if } \quad X_{v}<X_{w} \quad \bmod L_{\widetilde{\{v, w\}}} .
$$

It is not difficult to verify that $T$ is a linear order which satisfies Assertions $\mathrm{A} 1$ and $\mathrm{A} 2$.

In the next remark, we describe an important property of a traverse, which is often used.

Remark 4.3. Let $\sigma$ be a 2 -structure. As in the proof of Proposition 4.2, we associate with each strong clan $X$ of $\sigma$, which is not a limit of $\sigma$, a linear order $L_{X}$ defined on $\mathcal{G}$ allai $(\sigma[X])$. Moreover, whenever $\mathcal{Q u o t i e n t}_{\sigma}(X)$ is linear, we require that the arc set of $L_{X}$ is one of the two classes of $\mathcal{Q}$ uotient $\sigma(X)$.

Notation 4.4. In the sequel, $T$ denotes a traverse of $\sigma$ defined as in the proof of Proposition 4.2. 
Now, consider a strong clan $X$ of $\sigma$, which is not a limit of $\sigma$, such that $\mathcal{Q}^{\text {uotient }} \sigma(X)$ is linear. By Assertion A1, $\mathcal{G}$ allai $(\sigma[X])$ is a factorization of $T[X]$. Furthermore, it follows from the construction of $T$ that

$$
T[X] / \mathcal{G} \text { allai }(\sigma[X])=L_{X}
$$

By Assertion A2, the arc set of $L_{X}$ is a class of $\mathcal{Q}$ uotient $\sigma(X)$.

Notation 4.5. It follows from the definition of a quotient that there exists a class $e_{X}$ of $\sigma$ satisfying the following. Consider any $Y, Z \in \mathcal{G}$ allai $(\sigma[X])$ such that $(Y, Z) \in A\left(L_{X}\right)$. For $y \in Y$ and $z \in Z$, we have $(y, z) \in e_{X}$.

Therefore, for distinct $Y, Z \in \mathcal{G}$ allai $(\sigma[X])$, and for $y \in Y$ and $z \in Z$, the following assertions are equivalent.

- $y<z \bmod T$;

- $Y<Z \bmod T[X] / \mathcal{G}$ allai $(\sigma[X])$;

- $Y<Z \bmod L_{X}$;

- $(y, z) \in e_{X}$.

Notation 4.6. In the sequel, the linear order $T[X] / \mathcal{G}$ allai $(\sigma[X])$ (i.e. $\left.L_{X}\right)$ is denoted by $T_{X}$.

The next result follows from Remark 4.3.

Lemma 4.7. Let $\sigma$ be a 2-structure. Consider a strong clan $X$ of $\sigma$ such that $X$ is not a limit of $\sigma$, and $\mathcal{Q u o t i e n t}_{\sigma}(X)$ is linear. The following two assertions hold.

1. For each $F \subseteq \mathcal{G}$ allai $(\sigma[X]), F$ is an interval of $T_{X}$ if and only if $F$ is a clan of $\mathcal{Q}$ uotient $_{\sigma}(X)$.

2. Given $W \subseteq X$ such that $W \supseteq Y$ for some $Y \in \mathcal{G}$ allai $(\sigma[X]), W$ is an interval of $T$ if and only if $W$ is a clan of $\sigma$.

Proof. For the first assertion, recall that $T_{X}=L_{X}$ by Notation 4.6. By Assertion A2 (see Definition 4.1), the arc set of $L_{X}$ is one of the two classes of $\mathcal{Q}$ uotient $\sigma(X)$. Thus, the intervals of $L_{X}$ are exactly the clans of $\mathcal{Q u o t i e n t}_{\sigma}(X)$. Therefore, the first assertion holds.

For the second assertion, consider $W \subseteq X$ such that $W \supseteq Y$ for some $Y \in \mathcal{G}$ allai $(\sigma[X])$. Set

$$
\mathscr{W}=\{Z \in \mathcal{G} \text { allai }(\sigma[X]): Z \cap W \neq \emptyset\} .
$$

First, suppose that $W$ is an interval of $T$. Thus $W$ is an interval of $T[X]$. By Assertion A1, Gallai $(\sigma[X])$ is a factorization of $T[X]$. Since there exists 
$Y \in \mathcal{G}$ allai $(\sigma[X])$ such that $Y \subseteq W$, we obtain $W=\bigcup_{Z \in \mathscr{W}} Z$, and $\mathscr{W}$ is an interval of $T[X] / \mathcal{G}$ allai $(\sigma[X])$, that is, $T_{X}$. By the first assertion, $\mathscr{W}$ is a clan of $\mathcal{Q u o t i e n t}_{\sigma}(X)$. Hence $W$ is a clan of $\sigma[X]$ because $W=\bigcup_{Z \in \mathscr{W}} Z$. Since $X$ is a clan of $\sigma, W$ is a clan of $\sigma$.

Second, suppose that $W$ is a clan of $\sigma$. Thus $W$ is a clan of $\sigma[X]$. Since there exists $Y \in \mathcal{G}$ allai $(\sigma[X])$ such that $Y \subseteq W$, we obtain $W=\bigcup_{Z \in \mathscr{W}} Z$, and $\mathscr{W}$ is a clan of $\mathcal{Q u o t i e n t}_{\sigma}(X)$. By the first assertion, $\mathscr{W}$ is an interval of $T_{X}$, that is, $T[X] / \mathcal{G}$ allai $(\sigma[X])$. Since $\mathcal{G}$ allai $(\sigma[X])$ is a factorization of $T[X], \bigcup_{Z \in \mathscr{W}} Z$, that is, $W$ is an interval of $T[X]$. Hence $W$ is an interval of $T$ because $X$ is an interval of $T$ by Assertion A1.

The next result follows from Lemma 4.7 by using Proposition 3.3.

Corollary 4.8. Given a 2-structure $\sigma$, consider $L \in \mathcal{L}$ inear $(\sigma)$. For every $C \subseteq L, C$ is an interval of $T$ if and only if $C$ is a clan of $\sigma$.

The following is a simple consequence of Lemma 4.7. It generalizes [11, Theorem 2].

Corollary 4.9. Let $\sigma$ be an asymmetric 2-structure. All the clans of $\sigma$ are intervals of $T$.

Remark 4.10. In general, the opposite direction in Corollary 4.9 does not hold. For instance, consider the 2-structure $\sigma$ defined on $\{0,1,2\}$ by

$$
E(\sigma)=\{\{(0,1),(0,2)\},\{(1,0),(2,0)\},\{(1,2)\},\{(2,1)\}\} .
$$

Clearly $\{1,2\}$ is the only nontrivial clan of $\sigma$. Thus $\{1,2\}$ is the only nontrivial strong clan of $\sigma$. By Assertion A1 (see Definition 4.1), $\{1,2\}$ is an interval of each traverse of $\sigma$. Therefore $0<1<2,2<1<0,0<2<1$ and $1<2<0$ are the only traverses of $\sigma$. For $0<1<2$ and $2<1<0$, $\{0,1\}$ is an interval of the traverse which is not a clan of $\sigma$. For $0<2<1$ and $1<2<0,\{0,2\}$ is an interval of the traverse which is not a clan of $\sigma$.

The following notion of density is fundamental.

Definition 4.11. Let $L$ be a linear order. Recall that a subset $W$ of $V(L)$ is dense if $W$ intersects each interval $I$ of $L$ such that $|I| \geq 2$. A bicoloring $\beta: V(L) \longrightarrow\{0,1\}$ is dense if $\beta^{-1}(\{0\})$ and $\beta^{-1}(\{1\})$ are dense subsets.

Given a set $S$, consider $\mathcal{F} \subseteq 2^{S}$. The family $\mathcal{F}$ satisfies the Bernstein property [11] if there is $B \subseteq S$ such that for every $X \in \mathcal{F}, X$ intersects $B$ and $S \backslash B$. Clearly a linear order $L$ admits a dense bicoloring if and only if the family of the intervals of $L$ has the Bernstein property. 
Let $\sigma$ be a reversible 2 -structure such that $\mathcal{L}$ inear $(\sigma) \neq \emptyset$. Consider $L \in \mathcal{L}$ inear $(\sigma)$. To build a primitive extension of $\sigma$, we have to destroy all the nontrivial clans of $\sigma[L]$ by external elements. By Corollary 4.8, all the clans of $\sigma[L]$ are intervals of $T$. By the following proposition (see [11, Theorem 3]), $T$ admits a dense bicoloring, which allows us to destroy the nontrivial intervals of $T$.

Proposition 4.12 (Axiom of Choice). Every linear order admits a dense bicoloring.

In the next proposition, it is easy to verify that the second assertion implies the first. For the converse, see [12, Lemma 9].

Proposition 4.13. The following two assertions are equivalent:

1. every linear order admits a dense bicoloring;

2. every infinite linear order admits a primitive and faithful 1-extension, that is, a primitive 1-extension which is a tournament.

We complete the section with the following three results on primitive bounds. The first two follow from Propositions 4.12 and 4.13, and the derivation is omitted.

Corollary 4.14 (Erdős et al. [12]). For a linear order $L$, we have $p(L)=1$ or 2. Moreover, $p(L)=2$ if and only if $L$ is a finite linear order such that $|V(L)|$ is odd.

The next corollary is used in the proof of Theorem 7.2.

Corollary 4.15. Consider a reversible 2-structure $\sigma$ such that $|E(\sigma)| \geq 3$. Let $a \notin V(\sigma)$. For each $L \in \mathcal{L}$ inear $(\sigma)$, there is faithful extension $\tau$ of $\sigma$ defined on $V(\sigma) \cup\{a\}$ such that $\tau[L \cup\{a\}]$ is primitive.

The next theorem is used in the proof of Theorem 7.1.

Theorem 4.16. Let $\sigma$ be an asymmetric 2-structure. Suppose that $V(\sigma)$ is not a limit of $\sigma$, and $\mathcal{Q u o t i e n t}_{\sigma}(V(\sigma))$ is linear. If there exists $X \in \mathcal{G}$ allai $(\sigma)$ such that $|X| \geq 2$, then $p(\sigma)=1$.

Proof. Let $T$ be a traverse of $\sigma$ (see Notation 4.4). Consider the smallest interval $I$ of $T$ containing all the elements of $Y$ of $\mathcal{G}$ allai $(\sigma)$ such that $|Y| \geq 2$. Consider also the intervals

$$
\left\{\begin{array}{l}
I^{-}=\{v \in V(\sigma): v<i \quad \bmod T \text { for every } i \in I\} \\
\text { and } \\
I^{+}=\{v \in V(\sigma): i<v \quad \bmod T \text { for every } i \in I\} .
\end{array}\right.
$$


of $T$. It follows from the second assertion of Lemma 4.7 that $I, I^{-}$and $I^{+}$are clans of $\sigma$. By Proposition 4.12, $T$ admits a dense bicoloring $\beta$. We consider a bicoloration $\beta^{-}$of $T\left[I^{-}\right]$satisfying $\beta^{-}=\beta_{\left\lceil I^{-}\right.}$or $(1-\beta)_{\uparrow I^{-}}$and, when $T$ admits a smallest element $i^{-}$such that $i^{-} \in I^{-}$, we require that

$$
\beta^{-}\left(i^{-}\right)=1 \text {. }
$$

Similarly, we consider a bicoloration $\beta^{+}$of $T\left[I^{+}\right]$satisfying $\beta^{+}=\beta_{\left\lceil I^{+}\right.}$or $(1-\beta)_{I^{+}}$and, when $T$ admits a largest element $i^{+}$such that $i^{+} \in I^{+}$, we require that $\beta^{+}\left(i^{+}\right)=0$. Now consider the bicoloring $\beta^{\prime}$ of $T$ defined as follows. For every $v \in V(\sigma)$,

$$
\beta^{\prime}(v)=\left\{\begin{array}{l}
\beta^{-}(v) \text { if } v \in I^{-} \\
\beta(v) \text { if } v \in I \\
\beta^{+}(v) \text { if } v \in I^{+}
\end{array}\right.
$$

In general, $\beta^{\prime}$ may not be a dense bicoloring of $T$. But, for $J=I^{-}, I$ or $I^{+}$, we have

$$
\left(\beta^{\prime}\right)_{\lceil J} \text { is a dense bicoloring of } T[J] \text {. }
$$

We also have: for each clan $C$ of $\sigma$, with $|C| \geq 2$,

$$
\text { there exist } c, d \in C \text { such that } \beta^{\prime}(c) \neq \beta^{\prime}(d) .
$$

Indeed, consider a clan $C$ of $\sigma$ such that $|C| \geq 2$. For a contradiction, suppose that

$$
\left|C \cap I^{-}\right| \leq 1, \quad|C \cap I| \leq 1 \text { and }\left|C \cap I^{+}\right| \leq 1 .
$$

By Corollary 4.9, $C$ is an interval of $T$. Thus, if $C \cap I^{-} \neq \emptyset$ and $C \cap I^{+} \neq \emptyset$, then we have $I \subseteq C$ so that $X \subseteq C$, where $X \in \mathcal{G}$ allai $(\sigma)$ such that $|X| \geq 2$, which contradicts $|C \cap I| \leq 1$. For instance, we may assume that $C \cap I^{+}=\emptyset$. Since $|C| \geq 2$, we obtain $\left|C \cap I^{-}\right|=1$ and $|C \cap I|=1$. Therefore $C \backslash I \neq \emptyset$ and hence $I \backslash C$ is an interval of $T$. Suppose that there exists $Y \in \mathcal{G}$ allai $(\sigma)$ such that $|Y| \geq 2$ and $Y \nsubseteq I \backslash C$. Since $Y \subseteq I$, we have $Y \cap C \neq \emptyset$. Since $Y$ is a strong clan of $\sigma$, we obtain $Y \subseteq C$ or $C \subseteq Y$. We cannot have $Y \subseteq C$ because $Y \subseteq I$ and $|C \cap I|=1$. Furthermore, we cannot have $C \subseteq Y$ because $C \cap I^{-} \neq \emptyset$ and $Y \subseteq I$. It follows that for every $Y \in \mathcal{G}$ allai $(\sigma)$ such that $|Y| \geq 2$, we have $Y \subseteq I \backslash C$, which contradicts the minimality of $I$. Consequently, there exists $J=I^{-}, I$ or $I^{+}$such that $|C \cap J| \geq 2$. It follows from (4.2) that (4.3) holds. 
Set $e_{0}=e_{V(\sigma)}$ (see Notation 4.5) and $e_{1}=\left(e_{0}\right)^{\star}$. Let $a \notin V(\sigma)$. We consider the faithful extension $\tau$ of $\sigma$ defined on $V(\sigma) \cup\{a\}$ by

$$
(v, a)_{\tau}=e_{\beta^{\prime}(v)} \text { for every } v \in V(\sigma) .
$$

We prove that $\tau$ is primitive. Let $D$ be a clan of $\tau$ such that $|D| \geq 2$. We have to show that $D=V(\tau)$. It follows from (4.3) that $a \in D$, and

$$
\text { for each clan } C \text { of } \sigma \text { such that }|C| \geq 2, C \cap(D \backslash\{a\}) \neq \emptyset \text {. }
$$

Set

$$
\mathscr{D}=\{Y \in \mathcal{G} \text { allai }(\sigma): Y \cap(D \backslash\{a\}) \neq \emptyset\}
$$

By (4.4),

$$
\{Y \in \mathcal{G} \text { allai }(\sigma):|Y| \geq 2\} \subseteq \mathscr{D} .
$$

Thus $(D \backslash\{a\}) \backslash I^{-} \neq \emptyset$. Since $I^{-}$is a clan of $\sigma$ by Lemma 4.7, $I^{-} \backslash(D \backslash\{a\})$ is a clan of $\sigma$. For a contradiction, suppose that

$$
I^{-} \backslash(D \backslash\{a\}) \neq \emptyset \text {. }
$$

By (4.4), $\left|I^{-} \backslash(D \backslash\{a\})\right|=1$. Denote by $i^{-}$the unique element of $I^{-} \backslash$ $(D \backslash\{a\})$. Since $D \backslash\{a\}$ is an interval of $T$ by Corollary $4.9, i^{-}$is the smallest element of $T$. Let $X \in \mathcal{G}$ allai $(\sigma)$ such that $|X| \geq 2$. By (4.4), $X \cap(D \backslash\{a\}) \neq \emptyset$. Consider $x \in X \cap(D \backslash\{a\})$. Since $X \subseteq I, x \in I$ and hence $i^{-}<x \bmod T$. By Remark 4.3, $\left(i^{-}, x\right)_{\sigma}=e_{V(\sigma)}$, that is, $\left(i^{-}, x\right)_{\sigma}=e_{0}$. Since $\left(i^{-}, x\right)_{\tau}=\left(i^{-}, x\right)_{\sigma}$, we get $\left(i^{-}, x\right)_{\tau}=e_{0}$. Since $D$ is a clan of $\tau$, we have $\left(i^{-}, x\right)_{\tau}=\left(i^{-}, a\right)_{\tau}$. It follows that $\left(i^{-}, a\right)_{\tau}=e_{0}$. Therefore $\beta^{\prime}\left(i^{-}\right)=0$ and hence $\beta^{-}\left(i^{-}\right)=0$, which contradicts (4.1). Consequently, (4.6) does not hold, that is, $I^{-} \subseteq D \backslash\{a\}$. Similarly, we have $I^{+} \subseteq D \backslash\{a\}$.

Lastly, suppose for a contradiction that

$$
\text { there exists } Z \in \mathcal{G} \text { allai }(\sigma) \text { such that } D \backslash\{a\} \subsetneq Z \text {. }
$$

We get $\mathscr{D}=\{Z\}$. Since $\{Y \in \mathcal{G}$ allai $(\sigma):|Y| \geq 2\} \neq \emptyset$, it follows from (4.5) that $\{Y \in \mathcal{G}$ allai $(\sigma):|Y| \geq 2\}=\{Z\}$. Since $Z$ is an interval of $T$ by Corollary 4.9, we obtain $I=Z$. Furthermore, since $I^{-} \subseteq D \backslash\{a\}$ and $I^{+} \subseteq D \backslash\{a\}$, we get $I^{-}=\emptyset$ and $I^{+}=\emptyset$. It follows that $Z=V(\sigma)$, which is impossible because $Z \in \mathcal{G}$ allai $(\sigma)$. Therefore, (4.7) does not hold. Consequently

$$
D \backslash\{a\}=\bigcup_{Y \in \mathscr{D}} Y .
$$


By (4.5), $D \backslash\{a\} \supseteq Y$ for every $Y \in \mathcal{G}$ allai $(\sigma)$ such that $|Y| \geq 2$. Since $D \backslash\{a\}$ is an interval of $T$ by Corollary 4.9, it follows from the minimality of $I$ that $I \subseteq D \backslash\{a\}$. Finally, since $I^{-} \subseteq D \backslash\{a\}$ and $I^{+} \subseteq D \backslash\{a\}$, we get $D \backslash\{a\}=V(\sigma)$. Hence $D=V(\tau)$.

As a conclusion, $\tau$ is primitive. Thus $p(\sigma) \leq 1$. Since $\{Y \in \mathcal{G}$ allai $(\sigma)$ : $|Y| \geq 2\} \neq \emptyset, \sigma$ is imprimitive. Therefore $p(\sigma) \geq 1$, so $p(\sigma)=1$.

\section{Setting the stage}

In this section, as outlined in the overview (see page 547), we lay out some preliminary results on the construction of primitive faithful extensions.

Let $\sigma$ be an imprimitive and reversible 2-structure. To construct a primitive faithful extension of $\sigma$, we proceed as follows. First, we destroy locally the elements of $\mathcal{C}$ omplete $(\sigma)$ by using Lemmas 5.3 and 5.4 below. Second, we consider a suitable $\mathcal{F} \subseteq \mathcal{L}$ inear $(\sigma) \cup \mathcal{P}$ rimitive $(\sigma)$. To destroy the elements of $\mathcal{F} \cap \mathcal{L}$ inear $(\sigma)$, we use Corollary 4.15. To destroy the elements of $\mathcal{F} \cap \mathcal{P}$ rimitive $(\sigma)$, we use Lemma 5.5 below. Third, to destroy the other nontrivial clans of $\sigma$, we use dense bicolorations of a traverse of $\sigma$.

A remark on the construction of faithful extensions of reversible 2structures follows.

Remark 5.1. Consider reversible 2-structures $\sigma$ and $\sigma^{\prime}$ such that $V(\sigma) \cap$ $V\left(\sigma^{\prime}\right)=\emptyset$ with $\mid\left\{e^{\prime} \in E\left(\sigma^{\prime}\right): e^{\prime}\right.$ asymmetric $\}|\leq|\{e \in E(\sigma): e$ asymmetric $\} \mid$ and $\mid\left\{e^{\prime} \in E\left(\sigma^{\prime}\right): e^{\prime}\right.$ symmetric $\}|\leq|\{e \in E(\sigma): e$ symmetric $\} \mid$. There exists an injection $\iota: E\left(\sigma^{\prime}\right) \longrightarrow E(\sigma)$ such that $\iota\left(e^{\star}\right)=\iota(e)^{\star}$ for each $e \in E\left(\sigma^{\prime}\right)$. The function $\iota$ allows us to identify a class $e$ of $\sigma^{\prime}$ with the class $\iota(e)$ of $\sigma$. We have $\iota\left(\left\{e^{\prime} \in E\left(\sigma^{\prime}\right): e^{\prime}\right.\right.$ asymmetric $\left.\}\right) \subseteq\{e \in E(\sigma): e$ asymmetric $\}$ and $\iota\left(\left\{e^{\prime} \in E\left(\sigma^{\prime}\right): e^{\prime}\right.\right.$ symmetric $\left.\}\right) \subseteq\{e \in E(\sigma): e$ symmetric $\}$. Consider also a function $\varphi: V(\sigma) \times V\left(\sigma^{\prime}\right) \longrightarrow E(\sigma)$. It is easy to verify that there is a unique extension $\tau$ of $\sigma$ and $\sigma^{\prime}$ defined on $V(\sigma) \cup V\left(\sigma^{\prime}\right)$ which is a faithful extension of $\sigma$ such that $\sigma^{\prime} \hookrightarrow \tau=(\sigma \hookrightarrow \tau) \circ \iota$ and $\left(v, v^{\prime}\right)_{\tau}=((\sigma \hookrightarrow \tau) \circ \varphi)\left(v, v^{\prime}\right)$ for every $\left(v, v^{\prime}\right) \in V(\sigma) \times V\left(\sigma^{\prime}\right)$.

Notation 5.2. Let $\sigma$ be a 2-structure. We define the function $\vec{\sigma}$ as follows. For each $v \in V(\sigma)$,

$$
\begin{aligned}
\vec{\sigma}(v): \quad V(\sigma) \backslash\{v\} & \longrightarrow E(\sigma) \\
w & \longmapsto(v, w)_{\sigma} .
\end{aligned}
$$

The next lemma is used in the proof of Theorem 7.9 when we consider a reversible 2-structure $\sigma$ such that $|E(\sigma)| \geq 2$ and $\log _{|E(\sigma)|}(c(\sigma)) \geq \aleph_{0}$. 
Lemma 5.3. Let $\sigma$ be a reversible 2-structure such that $|E(\sigma)| \geq 2$. Consider a primitive, reversible and infinite 2-structure $\sigma^{\prime}$ such that

- $V(\sigma) \cap V\left(\sigma^{\prime}\right)=\emptyset$;

- $\mid\left\{e^{\prime} \in E\left(\sigma^{\prime}\right): e^{\prime}\right.$ asymmetric $\}|\leq|\{e \in E(\sigma): e$ asymmetric $\} \mid ;$

- $\mid\left\{e^{\prime} \in E\left(\sigma^{\prime}\right): e^{\prime}\right.$ symmetric $\}|\leq|\{e \in E(\sigma): e$ symmetric $\} \mid$;

- $|E(\sigma)|<|E(\sigma)|^{\left|V\left(\sigma^{\prime}\right)\right|}$.

For each $S \subseteq V(\sigma)$ such that $|S| \leq|E(\sigma)|^{\left|V\left(\sigma^{\prime}\right)\right|}$, there exists an extension $\tau$ of $\sigma$ and $\sigma^{\prime}$ to $V(\sigma) \cup V\left(\sigma^{\prime}\right)$ such that $\tau$ is a faithful extension of $\sigma$ and $\tau\left[S \cup V\left(\sigma^{\prime}\right)\right]$ is primitive.

Proof. Since $\mid\left\{e^{\prime} \in E\left(\sigma^{\prime}\right): e^{\prime}\right.$ asymmetric $\}|\leq|\{e \in E(\sigma): e$ asymmetric $\} \mid$ and $\mid\left\{e^{\prime} \in E\left(\sigma^{\prime}\right): e^{\prime}\right.$ symmetric $\}|\leq|\{e \in E(\sigma): e$ symmetric $\} \mid$, there exists an injection $\iota: E\left(\sigma^{\prime}\right) \longrightarrow E(\sigma)$ such that $\iota\left(e^{\star}\right)=\iota(e)^{\star}$ for every $\left.e \in E_{(} \sigma^{\prime}\right)$. Thus $\iota\left(\left\{e^{\prime} \in E\left(\sigma^{\prime}\right): e^{\prime}\right.\right.$ asymmetric $\left.\}\right) \subseteq\{e \in E(\sigma): e$ asymmetric $\}$ and $\iota\left(\left\{e^{\prime} \in E\left(\sigma^{\prime}\right): e^{\prime}\right.\right.$ symmetric $\left.\}\right) \subseteq\{e \in E(\sigma): e$ symmetric $\}$. In what follows, we identify $e^{\prime} \in E\left(\sigma^{\prime}\right)$ with $\iota\left(e^{\prime}\right) \in E(\sigma)$.

Let $v^{\prime} \in V\left(\sigma^{\prime}\right)$. We have $\overrightarrow{\sigma^{\prime}}\left(v^{\prime}\right): V\left(\sigma^{\prime}\right) \backslash\left\{v^{\prime}\right\} \longrightarrow E(\sigma)$. Denote by $\mathbb{F}_{v^{\prime}}$ the family of the extensions of $\overrightarrow{\sigma^{\prime}}\left(v^{\prime}\right)$ to $V\left(\sigma^{\prime}\right)$. For each $v^{\prime} \in V\left(\sigma^{\prime}\right)$, we have $\left|\mathbb{F}_{v^{\prime}}\right|=|E(\sigma)|$. Also, for each $e \in E(\sigma)$, consider the function $\bar{e}: V\left(\sigma^{\prime}\right) \longrightarrow\{e\}$ defined by $\bar{e}\left(v^{\prime}\right)=e$ for every $v^{\prime} \in V\left(\sigma^{\prime}\right)$. We obtain

$$
\begin{aligned}
\left|\left(\bigcup_{v^{\prime} \in V\left(\sigma^{\prime}\right)} \mathbb{F}_{v^{\prime}}\right) \cup\{\bar{e}: e \in E(\sigma)\}\right| & \leq\left|V\left(\sigma^{\prime}\right)\right| \times|E(\sigma)|+|E(\sigma)| \\
& =\max \left(|E(\sigma)|,\left|V\left(\sigma^{\prime}\right)\right|\right) .
\end{aligned}
$$

Since $|E(\sigma)| \geq 2,|E(\sigma)|^{\left|V\left(\sigma^{\prime}\right)\right|}>\left|V\left(\sigma^{\prime}\right)\right|$. Furthermore, since $|E(\sigma)|^{\left|V\left(\sigma^{\prime}\right)\right|}>$ $|E(\sigma)|$, we get $|E(\sigma)|^{\left|V\left(\sigma^{\prime}\right)\right|}>\max \left(|E(\sigma)|,\left|V\left(\sigma^{\prime}\right)\right|\right)$. Therefore

$$
\left|E(\sigma)^{V\left(\sigma^{\prime}\right)} \backslash\left(\left(\bigcup_{v^{\prime} \in V\left(\sigma^{\prime}\right)} \mathbb{F}_{v^{\prime}}\right) \cup\{\bar{e}: e \in E(\sigma)\}\right)\right|=|E(\sigma)|^{\left|V\left(\sigma^{\prime}\right)\right|} .
$$

Since $|S| \leq|E(\sigma)|^{\left|V\left(\sigma^{\prime}\right)\right|}$, there exists an injection

$$
A: S \longrightarrow E(\sigma)^{V\left(\sigma^{\prime}\right)} \backslash\left(\left(\bigcup_{v^{\prime} \in V\left(\sigma^{\prime}\right)} \mathbb{F}_{v^{\prime}}\right) \cup\{\bar{e}: e \in E(\sigma)\}\right) .
$$

Now, consider the faithful extension $\tau$ of $\sigma$ defined on $V(\sigma) \cup V\left(\sigma^{\prime}\right)$ as follows. 
Let $e \in E(\sigma)$. Given distinct $u, v \in V(\sigma) \cup V\left(\sigma^{\prime}\right)$,

$$
(u, v)_{\tau}=\left\{\begin{array}{l}
(u, v)_{\sigma} \text { if } u, v \in V(\sigma), \\
(u, v)_{\sigma^{\prime}} \text { if } u, v \in V\left(\sigma^{\prime}\right), \\
A(u)(v) \text { if } u \in S \text { and } v \in V\left(\sigma^{\prime}\right), \\
e \text { if } u \in V(\sigma) \backslash S \text { and } v \in V\left(\sigma^{\prime}\right) .
\end{array}\right.
$$

It is not difficult to verify that $\tau\left[S \cup V\left(\sigma^{\prime}\right)\right]$ is primitive.

The next lemma is used in the proof of Theorem 7.4 when we consider a reversible 2 -structure $\sigma$ such that $2 \leq|E(\sigma)| \leq c(\sigma)<\aleph_{0}$.

Lemma 5.4. Let $\sigma$ be a reversible 2-structure such that $|E(\sigma)| \geq 2$. Consider $S \subseteq V(\sigma)$ such that $2 \leq|E(\sigma)| \leq|S|<\aleph_{0}$, and $\sigma[S]$ is complete. Let $e$ be the symmetric class of $\sigma$ such that $(s, t) \in e$ for distinct $s, t \in S$. Let $S^{\prime}$ be a set such that $S^{\prime} \cap V(\sigma)=\emptyset$ and $\left|S^{\prime}\right|=\left\lceil\log _{|E(\sigma)|}(|S|+1)\right\rceil$. There exists a faithful extension $\tau$ of $\sigma$ defined on $V(\sigma) \cup S^{\prime}$ satisfying

1. for every $s^{\prime} \in S^{\prime}$, there is $s \in S$ such that $(\vec{\tau}(s))^{-1}(\{e\}) \cap S^{\prime}=\left\{s^{\prime}\right\}$;

2. $\tau\left[S \cup S^{\prime}\right]$ is primitive.

Proof. Since $S$ is finite, $S^{\prime}$ is also and

$$
|E(\sigma)|^{\left|S^{\prime}\right|-1} \leq|S|<|E(\sigma)|^{\left|S^{\prime}\right|} .
$$

Furthermore, since $|S|+1>|E(\sigma)|$ and $\left|S^{\prime}\right|=\left\lceil\log _{|E(\sigma)|}(|S|+1)\right\rceil$, we have $\left|S^{\prime}\right| \geq 2$. Therefore $|E(\sigma)|^{\left|S^{\prime}\right|-1} \geq\left|S^{\prime}\right|$, and hence $\left|S^{\prime}\right| \leq|S|$. Thus there exists an injection $\varphi: S^{\prime} \longrightarrow S$. Since $|E(\sigma)| \geq 2$, there exists $f \in E(\sigma) \backslash\{e\}$. Consider $A: \varphi\left(S^{\prime}\right) \longrightarrow E(\sigma)^{S^{\prime}}$ defined as follows. For each $s^{\prime} \in S^{\prime}$,

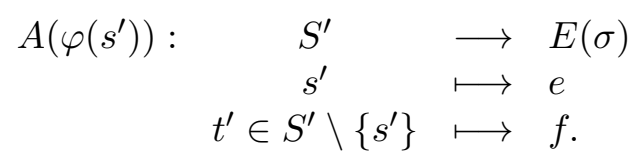

Consider the function $\bar{e}: S^{\prime} \longrightarrow\{e\}$ defined by $\bar{e}\left(s^{\prime}\right)=e$ for every $s^{\prime} \in S^{\prime}$. Since $\left|S^{\prime}\right| \geq 2$, we have $A: \varphi\left(S^{\prime}\right) \longrightarrow E(\sigma)^{S^{\prime}} \backslash\{\bar{e}\}$. Therefore the function $A$ is injective. Since $|S|+1 \leq|E(\sigma)|^{\left|S^{\prime}\right|}$, there exists an injection $B: S \longrightarrow$ $E(\sigma)^{S^{\prime}} \backslash\{\bar{e}\}$ such that $B_{\left\lceil\varphi\left(S^{\prime}\right)\right.}=A$. We consider a faithful extension $\tau$ of $\sigma$ defined on $V(\sigma) \cup S^{\prime}$ satisfying

- $\left(s^{\prime}, t^{\prime}\right)_{\tau}=f$ or $f^{\star}$ for distinct $s^{\prime}, t^{\prime} \in S^{\prime}$, 
- $\vec{\tau}(s)_{\uparrow S^{\prime}}=B(s)$ for each $s \in S$.

For every $s^{\prime} \in S^{\prime},\left(\vec{\tau}\left(\varphi\left(s^{\prime}\right)\right)\right)^{-1}(\{e\}) \cap S^{\prime}=\left\{s^{\prime}\right\}$, and it is simple to verify that $\tau\left[S \cup S^{\prime}\right]$ is primitive.

Let $\sigma$ be a reversible 2 -structure such that $\mathcal{P}$ rimitive $(\sigma) \neq \emptyset$. Consider $\mathcal{F} \subseteq \mathcal{P}$ rimitive $(\sigma)$. When $\sigma$ is asymmetric and $|E(\sigma)|=2$, that is, $\sigma$ is a tournament, we require that for every $X \in \mathcal{F},|X| \geq 5$. By using Theorem 3.7 and the next lemma, we can construct a faithful extension $\tau$ of $\sigma$ to $V(\sigma) \cup$ $\{a\}$ (where $a \notin V(\sigma))$ such that $\tau[P \cup\{a\}]$ is primitive for every $P \in \mathcal{F}$. Indeed, consider $P \in \mathcal{F}$. By the following lemma, there exists a faithful extension $\tau_{P}$ of $\sigma[P]$ to $P \cup\{a\}$ which is primitive. Furthermore, it follows from Theorem 3.7 that the elements of $\mathcal{F}$ are pairwise disjoint. Therefore, there exists a faithful extension $\tau$ of $\sigma$ to $V(\sigma) \cup\{a\}$ such that $\tau[P \cup\{a\}]=\tau_{P}$ for every $P \in \mathcal{F}$.

Lemma 5.5. Consider a primitive and reversible 2-structure $\sigma$. Let a $\notin$ $V(\sigma)$. If $V(\sigma)$ is finite, then $\sigma$ admits $|E(\sigma)|^{|V(\sigma)|}-|E(\sigma)| \times|V(\sigma)|-|E(\sigma)|$ primitive and faithful extensions of $\sigma$ to $V(\sigma) \cup\{a\}$. If $V(\sigma)$ is infinite, then $\sigma$ admits $|E(\sigma)|^{|V(\sigma)|}$ such extensions.

Proof. Consider a faithful extension $\tau$ of $\sigma$ to $V(\sigma) \cup\{a\}$. Assume that $\tau$ is imprimitive, and consider a nontrivial clan $C$ of $\tau$. Since $\sigma$ is primitive, we have $|C \cap V(\sigma)| \leq 1$ or $C \cap V(\sigma)=V(\sigma)$. Since $C$ is a nontrivial clan of $\tau,|C| \geq 2$ and hence $|C \cap V(\sigma)| \geq 1$. By the same, $C \subsetneq V(\tau)$. Therefore, we obtain that either there is $v \in V(\sigma)$ such that $C=\{a, v\}$ or $C=$ $V(\sigma)$. In the second instance, $\vec{\tau}(a)$ is constant. In the first, $\vec{\tau}(a)_{\mid V(\sigma) \backslash\{v\}}=$ $\vec{\sigma}(v)$. Conversely, if $\vec{\tau}(a)$ is constant, then $V(\sigma)$ is a nontrivial clan of $\tau$. Furthermore, if there is $v \in V(\sigma)$ such that $\vec{\tau}(a)_{\mid V(\sigma) \backslash\{v\}}=\vec{\sigma}(v)$, then $\{a, v\}$ is a nontrivial clan of $\tau$.

Consequently, we obtain the following. Given a faithful extension $\tau$ of $\sigma$ to $V(\sigma) \cup\{a\}, \tau$ is imprimitive if and only if either $\vec{\tau}(a)$ is constant or there is $v \in V(\sigma)$ such that $\vec{\tau}(a)_{\mid V(\sigma) \backslash\{v\}}=\vec{\sigma}(v)$. Therefore $\sigma$ admits $|E(\sigma)| \times$ $|V(\sigma)|+|E(\sigma)|$ imprimitive and faithful extensions to $V(\sigma) \cup\{a\}$. If $V(\sigma)$ is finite, then $|E(\sigma)|$ is also and $\sigma$ admits $|E(\sigma)|^{|V(\sigma)|}-|E(\sigma)| \times|V(\sigma)|-|E(\sigma)|$ primitive and faithful extensions of $\sigma$ to $V(\sigma) \cup\{a\}$. Suppose that $V(\sigma)$ is infinite. We get $|E(\sigma)| \leq|V(\sigma)|$ and hence $|E(\sigma)| \times|V(\sigma)|+|E(\sigma)|=|V(\sigma)|$. Since $\sigma$ is primitive, $|E(\sigma)| \geq 2$. Thus $|V(\sigma)|<|E(\sigma)|^{|V(\sigma)|}$. Consequently, $\sigma$ admits $|E(\sigma)|^{|V(\sigma)|}$ primitive and faithful extensions of $\sigma$ to $V(\sigma) \cup\{a\}$.

The rather technical appearing conditions of the following two results permit their use in different ways throughout Section 7. The usefulness of 
dense bicolorings of traverses appears in the first one. We use the following notation.

Notation 5.6. Let $\sigma$ be a 2-structure. For convenience, set

- $C(\sigma)=\bigcup_{C \in \mathcal{C} \text { omplete }(\sigma)} C$,

- $L(\sigma)=\bigcup_{L \in \mathcal{L} \text { inear }(\sigma)} L$,

- $P(\sigma)=\bigcup_{P \in \mathcal{P} \text { rimitive }(\sigma)} P$.

Lemma 5.7. Let $\sigma$ be a reversible 2-structure such that $|E(\sigma)| \geq 2$. Consider a traverse $T$ of $\sigma$ and a dense bicoloring $\beta$ of $T$. Let $S^{\prime}$ be a set such that $S^{\prime} \neq \emptyset$ and $S^{\prime} \cap V(\sigma)=\emptyset$. Consider distinct $A_{0}, A_{1} \in E(\sigma)^{S^{\prime}}$. Also let $\mathcal{F} \subseteq \mathcal{L}$ inear $(\sigma) \cup \mathcal{P}$ rimitive $(\sigma)$. Consider a faithful extension $\tau$ of $\sigma$ to $V(\sigma) \cup S^{\prime}$ satisfying

$$
\begin{aligned}
& \text { for each } C \in \mathcal{C} \text { omplete }(\sigma) \text {, } \\
& \qquad\{D \in \mathcal{C} \operatorname{lans}(\sigma[C]):|D| \geq 2\} \cap \mathcal{C} \operatorname{lans}(\tau)=\emptyset ;
\end{aligned}
$$

for each $X \in \mathcal{F}, \tau\left[X \cup S^{\prime}\right]$ is primitive;

for each $v \in V(\sigma) \backslash\left(C(\sigma) \cup\left(\bigcup_{X \in \mathcal{F}} X\right)\right), \vec{\tau}(v)_{\left\lceil S^{\prime}\right.}=A_{\beta(v)}$.

Then, we have

$$
\{C \in \mathcal{C} \operatorname{lans}(\sigma):|C| \geq 2\} \cap \mathcal{C} \operatorname{lans}(\tau)=\emptyset .
$$

Proof. To begin, given $C \in \mathcal{C}$ omplete $(\sigma) \cup \mathcal{L}$ inear $(\sigma) \cup \mathcal{P}$ rimitive $(\sigma)$, we prove that

$$
\{D \in \mathcal{C} \operatorname{lans}(\sigma[C]):|D| \geq 2\} \cap \mathcal{C} \operatorname{lans}(\tau)=\emptyset .
$$

Clearly (5.2) follows from (5.1a) when $C \in \mathcal{C}$ omplete $(\sigma)$. Suppose that $C \in$ $\mathcal{L}$ inear $(\sigma) \cup \mathcal{P} \operatorname{rimitive}(\sigma)$, and consider a clan $D$ of $\sigma[C]$ such that $|D| \geq 2$. We have to prove that $D$ is not a clan of $\tau$. If $C \in \mathcal{F}$, then $\tau\left[C \cup S^{\prime}\right]$ is primitive by (5.1b). Thus $D$ is not a clan of $\tau\left[C \cup S^{\prime}\right]$, and hence $D$ is not a clan of $\tau$. Suppose that $C \in(\mathcal{L}$ inear $(\sigma) \cup \mathcal{P}$ rimitive $(\sigma)) \backslash \mathcal{F}$. We show that $D$ is an interval of $T$. By Corollary 4.8, if $C \in \mathcal{L}$ inear $(\sigma)$, then $D$ is an interval of $T$. If $C \in \mathcal{P} \operatorname{rimitive}(\sigma)$, then $D=C$, and $C$ is a strong clan of $\sigma$ because $\sigma[C]$ is primitive. By Assertion A1 (see Definition 4.1), $D$ is an interval of $T$. By (5.1c), since $D \subseteq V(\sigma) \backslash\left(C(\sigma) \cup\left(\bigcup_{X \in \mathcal{F}} X\right)\right)$, we have $\vec{\tau}(v)_{\mid S^{\prime}}=A_{\beta(v)}$ for every $v \in D$. Since $\beta$ is a dense bicoloring of $T$, there exist distinct $u, v \in D$ such that $\beta(u) \neq \beta(v)$. Therefore $A_{\beta(u)} \neq A_{\beta(v)}$ and $\vec{\tau}(u)_{\left\lceil S^{\prime}\right.} \neq \vec{\tau}(v)_{\left\lceil S^{\prime}\right.}$ by 
(5.1c). Consequently there is $s^{\prime} \in S^{\prime}$ such that $\left(u, s^{\prime}\right)_{\tau} \neq\left(v, s^{\prime}\right)_{\tau}$ so that $D$ is not a clan of $\tau$. It follows that (5.2) holds.

To continue we show that

$$
\{X \in \mathcal{S} \operatorname{trong}(\sigma):|X| \geq 2\} \cap \mathcal{C} \operatorname{lans}(\tau)=\emptyset .
$$

Given a strong clan $X$ of $\sigma$ such that $|X| \geq 2$, we distinguish the following two cases to show that $X$ is not a clan of $\tau$.

1. Suppose that there exists $C \in \mathcal{C}$ omplete $(\sigma) \cup \mathcal{L}$ inear $(\sigma) \cup \mathcal{P}$ rimitive $(\sigma)$ such that $X \cap C \neq \emptyset$. Since $X$ is a strong clan of $\sigma, X \subseteq C$ or $C \subseteq X$. Thus $X \cap C=X$ or $C$ and hence $X \cap C$ is a clan of $\sigma[C]$ such that $|X \cap C| \geq 2$. It follows from (5.2) that there is $s^{\prime} \in S^{\prime}$ such that $s^{\prime} \nLeftarrow{ }_{\tau} X \cap C$. Therefore $s^{\prime} \nLeftarrow{ }_{\tau} X$ and $X$ is not a clan of $\tau$.

2. Suppose that $X \cap(C(\sigma) \cup L(\sigma) \cup P(\sigma))=\emptyset$. By Assertion A1 (see Definition 4.1), $X$ is an interval of $T$. Since $\beta$ is a dense bicoloring of $T$, there exist distinct $x, y \in X$ such that $\beta(x) \neq \beta(y)$. Thus $A_{\beta(x)} \neq A_{\beta(y)}$ and $\vec{\tau}(x)_{\left\lceil S^{\prime}\right.} \neq \vec{\tau}(y)_{\left\lceil S^{\prime}\right.}$ by (5.1c). Consequently there is $s^{\prime} \in S^{\prime}$ such that $\left(x, s^{\prime}\right)_{\tau} \neq\left(y, s^{\prime}\right)_{\tau}$ so that $X$ is not a clan of $\tau$.

It follows that (5.3) holds.

To conclude, consider a clan $C$ of $\sigma$ such that $|C| \geq 2$. We must show that $C$ is not a clan of $\tau$. By (5.3), we can suppose that $C$ is not a strong clan of $\sigma$. By Lemma 2.10, $\widetilde{C}$ is a strong clan of $\sigma$ which is not a limit of $\sigma$, and $C=\bigcup_{X \in \mathscr{C}} X$, where $\mathscr{C}$ is a nontrivial clan of $\mathcal{Q u o t i e n t}_{\sigma}(\widetilde{C})$. We distinguish the following two cases. First, suppose that there exists $X \in \mathscr{C}$ such that $|X| \geq 2$. Since $X$ is a strong clan of $\sigma, X$ is not a clan of $\tau$ by (5.3). Since $X \subseteq C, C$ is not a clan of $\tau$. Second, suppose that $|X|=1$ for each $X \in \mathscr{C}$. Hence $\{v\} \in \mathcal{Q u o t i e n t}_{\sigma}(\widetilde{C})$ for every $v \in C$. By maximality of elements of $\mathcal{Q u o t i e n t}_{\sigma}(\widetilde{C}), \widehat{\{v\}}=\widetilde{C}$ for every $v \in C$. We obtain that $v \simeq_{\sigma} w$ for any $v, w \in C$. Thus there is $D \in\left(V(\sigma) / \simeq_{\sigma}\right)$ such that $|D| \geq 2$ and $D \supseteq C$. By Theorem 3.7, $D \in \mathcal{C}$ omplete $(\sigma) \cup \mathcal{L}$ inear $(\sigma) \cup \mathcal{P}$ rimitive $(\sigma)$. By (5.2), $C$ is not a clan of $\tau$.

The following result is a simple consequence of Lemma 5.7 when only 1-extensions are considered.

Corollary 5.8. Let $\sigma$ be a reversible 2-structure such that $|E(\sigma)| \geq 2$. Consider a traverse $T$ of $\sigma$ and a dense bicoloring $\beta$ of $T$. Let $a \notin V(\sigma)$. Consider distinct $e_{0}, e_{1} \in E(\sigma)$. Also let $\mathcal{F} \subseteq \mathcal{L}$ inear $(\sigma) \cup \mathcal{P}$ rimitive $(\sigma)$. Consider a faithful extension $\tau$ of $\sigma$ to $V(\sigma) \cup\{a\}$ satisfying

- for each $C \in \mathcal{C}$ omplete $(\sigma),\{D \in \mathcal{C} \operatorname{lans}(\sigma[C]):|D| \geq 2\} \cap \mathcal{C} \operatorname{lans}(\tau)=\emptyset$; 
- for each $X \in \mathcal{F}, \tau[X \cup\{a\}]$ is primitive;

- for each $v \in V(\sigma) \backslash\left(C(\sigma) \cup\left(\bigcup_{X \in \mathcal{F}} X\right)\right),(v, a)_{\tau}=e_{\beta(v)}$.

Then, for each clan $D_{\tau}$ of $\tau$ such that $\left|D_{\tau}\right| \geq 2$, the following assertions hold

1. $a \in D_{\tau}$;

2. for every clan $C$ of $\sigma$ such that $|C| \geq 2$, we have $C \cap\left(D_{\tau} \backslash\{a\}\right) \neq \emptyset$;

3. for every clan $C$ of $\sigma$ such that $|C| \geq 2$ and $\tau[C \cup\{a\}]$ is primitive, we have $C \subseteq D_{\tau} \backslash\{a\}$; in particular $C \subseteq D_{\tau} \backslash\{a\}$ for each $C \in \mathcal{F}$.

\section{Inclusive clans of a 2-structure}

Consider reversible 2-structures $\sigma$ and $\tau$ as in Corollary 5.8. Consider also $\mathcal{F} \subseteq \mathcal{L}$ inear $(\sigma) \cup \mathcal{P}$ rimitive $(\sigma)$. Suppose that $\tau$ is imprimitive, and consider a nontrivial clan $D_{\tau}$ of $\tau$. Clearly $D_{\tau} \backslash\{a\}$ is a clan of $\sigma$. By Corollary 5.8, $C \cap\left(D_{\tau} \backslash\{a\}\right) \neq \emptyset$ for every clan $C$ of $\sigma$ such that $|C| \geq 2$. Moreover, $C \subseteq D_{\tau} \backslash\{a\}$ for each $C \in \mathcal{F}$. This leads us to introduce the following notion.

Definition 6.1. Given a 2-structure $\sigma$, a clan $C$ of $\sigma$ is inclusive if $C(\sigma) \cup$ $L(\sigma) \cup P(\sigma) \subseteq C$, and $C \cap X \neq \emptyset$ for each strong clan $X$ of $\sigma$ such that $|X| \geq 2$.

Let $\sigma$ be a 2-structure. Obviously, $V(\sigma)$ is an inclusive clan of $\sigma$, and $J \neq \emptyset$ for every inclusive clan $J$ of $\sigma$. Furthermore, given an inclusive clan $J$ of $\sigma$, and a clan $C$ of $\sigma$, we have

$$
\text { if } J \subseteq C \text {, then } C \text { is an inclusive clan of } \sigma \text {. }
$$

We begin with three lemmas.

Lemma 6.2. Let $\sigma$ be a 2-structure. For each inclusive clan $J$ of $\sigma, V_{\downarrow}(\sigma) \subseteq$ $J$.

Proof. Let $v \in V_{\downarrow}(\sigma)$. We show that $v \in J$. Set

$$
\mathcal{F}_{v}=\{X \in \mathcal{S} \operatorname{trong}(\sigma): v \in X,|X| \geq 2\} .
$$

Since $v \in V_{\downarrow}(\sigma)$, we have

$$
\bigcap_{X \in \mathcal{F}_{v}} X=\{v\} .
$$

Since $J$ is an inclusive clan of $\sigma, J \cap X \neq \emptyset$ for every $X \in \mathcal{F}_{v}$. Thus, for every $X \in \mathcal{F}_{v}$, we have $X \subseteq J$ or $J \subseteq X$. If there exists $X \in \mathcal{F}_{v}$ such that $X \subseteq J$, 
then $v \in J$. Therefore, suppose that $J \subseteq X$ for every $X \in \mathcal{F}_{v}$. It follows that $J \subseteq\{v\}$. Since $J$ is an inclusive clan of $\sigma, J \neq \emptyset$. Hence $v \in J$.

Lemma 6.3. Let $\sigma$ be a 2-structure. If there exists $v \in V(\sigma)$ such that $\{v\}$ is an inclusive clan of $\sigma$, then $\left(V(\sigma) / \simeq_{\sigma}\right)=V_{1}(\sigma), V_{\downarrow}(\sigma)=\{v\}$, and $\{v\}$ is the smallest inclusive clan of $\sigma$ under inclusion.

Proof. By Theorem 3.7, $\left\{D \in\left(V(\sigma) / \simeq_{\sigma}\right):|D| \geq 2\right\}=\mathcal{C}$ omplete $(\sigma) \cup$ $\mathcal{L}$ inear $(\sigma) \cup \mathcal{P}$ rimitive $(\sigma)$. Since $C(\sigma) \cup L(\sigma) \cup P(\sigma) \subseteq\{v\}$, we obtain

$$
\left(V(\sigma) / \simeq_{\sigma}\right)=V_{1}(\sigma) .
$$

Now, we prove that $v \in V_{\downarrow}(\sigma)$. Suppose for a contradiction that $|\widehat{\{v\}}| \geq 2$. By Lemma 2.11, $\widehat{\{v\}}$ is not a limit of $\sigma$, and $\{v\} \in \mathcal{Q}_{\text {uotient }}(\widehat{\{v\}})$. Moreover, since $\{v\} \cap X \neq \emptyset$ for each $X \in \mathcal{Q u o t i e n t}_{\sigma}(\widehat{\{v\}})$ such that $|X| \geq 2$, we

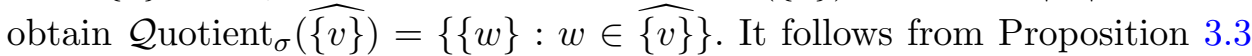
that $\widehat{\{v\}} \in \mathcal{C}$ omplete $(\sigma) \cup \mathcal{L}$ inear $(\sigma) \cup \mathcal{P}$ rimitive $(\sigma)$. By Theorem 3.7, $\widehat{\{v\}} \in$ $\left\{D \in\left(V(\sigma) / \simeq_{\sigma}\right):|D| \geq 2\right\}$, contradicts (6.2). Consequently $\widehat{\{v\}}=\{v\}$, that is, $v \in V_{\downarrow}(\sigma)$. It follows from Lemma 6.2 that $V_{\downarrow}(\sigma)=\{v\}$. Moreover, by Lemma $6.2, V_{\downarrow}(\sigma) \subseteq J$ for each inclusive clan $J$ of $\sigma$. Therefore, $\{v\}$ is the smallest inclusive clan of $\sigma$ under inclusion.

Lemma 6.4. Let $\sigma$ be a 2-structure. Given an inclusive clan $J$ of $\sigma$, we have $J \cap C \neq \emptyset$ for every clan $C$ of $\sigma$ such that $|C| \geq 2$.

Proof. By definition of an inclusive clan, we can consider a non-strong clan $C$ of $\sigma$ such that $|C| \geq 2$. By Lemma 2.10, $\widetilde{C}$ is not a limit of $\sigma$, and there exists a nontrivial clan $\mathscr{C}$ of $\mathcal{Q u o t i e n t}_{\sigma}(\widetilde{C})$ such that $C=\bigcup_{X \in \mathscr{C}} X$. If there exists $X \in \mathscr{C} \cap \mathcal{Q u o t i e n t}_{\sigma}(\widetilde{C})$ such that $|X| \geq 2$, then $J \cap X \neq \emptyset$, and hence $J \cap C \neq$ $\emptyset$. Thus, suppose that $|X|=1$ for every $X \in \mathscr{C}$. We obtain that $v \simeq_{\sigma} w$ for any $v, w \in C$. Therefore, there exists $D \in\left(V(\sigma) / \simeq_{\sigma}\right)$ such that $|D| \geq 2$ and $D \supseteq C$. By Theorem 3.7, $D \in \mathcal{C}$ omplete $(\sigma) \cup \mathcal{L}$ inear $(\sigma) \cup \mathcal{P}$ rimitive $(\sigma)$. Therefore $D \subseteq J$, and $C \subseteq J$.

The next two propositions are used in the proof of Theorem 6.7. The first one provides two interesting properties of the set of inclusive clans.

Proposition 6.5. Let $\sigma$ be a 2-structure. The set of inclusive clans of $\sigma$ is closed under intersection and union.

Proof. Let $J$ and $K$ be inclusive clans of $\sigma$. To begin, we show that $J \cap K \neq \emptyset$. If $|J|=|K|=1$, then it follows from Lemma 6.3 that $J=K=V_{\downarrow}(\sigma)$. Hence suppose for example that $|J| \geq 2$. By Lemma $6.4, J \cap K \neq \emptyset$. 
Since $J \cap K \neq \emptyset, J \cup K$ is a clan of $\sigma$ and hence $J \cup K$ is an inclusive clan of $\sigma$ by (6.1).

Lastly, we prove that $J \cap K$ is an inclusive clan of $\sigma$. Since $C(\sigma) \cup L(\sigma) \cup$ $P(\sigma) \subseteq J$ and $C(\sigma) \cup L(\sigma) \cup P(\sigma) \subseteq K$, we have $C(\sigma) \cup L(\sigma) \cup P(\sigma) \subseteq J \cap K$. Now consider a strong clan $X$ of $\sigma$ such that $|X| \geq 2$. We have $J \cap X \neq \emptyset$ and $K \cap X \neq \emptyset$. Thus $J, X$ and $K, X$ are comparable under inclusion. We obtain $J \cap K \subseteq X$ or $X \subseteq J \cap K$.

The next result provides a structural analysis of non-prime and inclusive clans.

Proposition 6.6. Let $\sigma$ be a 2-structure. Consider a traverse $T$ of $\sigma$. Given an inclusive clan $J$ of $\sigma$, if $J$ is not a strong clan of $\sigma$, then $\mathcal{Q u o t i e n t}_{\sigma}(\widetilde{J})$ is complete or linear. Moreover, the following assertions hold.

1. Suppose that $\mathcal{Q u o t i e n t}_{\sigma}(\widetilde{J})$ is complete. We obtain

(a) there exists $j \in \widetilde{J}$ such that $\{v \in \widetilde{J}:\{v\} \in \mathcal{G}$ allai $(\sigma[\widetilde{J}])\}=\{j\}$;

(b) $J=\widetilde{J} \backslash\{j\}$ and hence

$$
J=\bigcup_{X \in \mathcal{G} \text { allai }(\sigma[\widetilde{J}]),|X| \geq 2} X .
$$

2. Suppose that $\operatorname{Quotient}_{\sigma}(\widetilde{J})$ is linear. Denote by I the smallest interval of $T[\widetilde{J}]$ containing all the elements $X$ of $\mathcal{G}$ allai $(\sigma[\widetilde{J}])$ such that $|X| \geq 2$. Also consider $I^{-}=\{j \in \widetilde{J}: j<i \bmod T$ for every $i \in I\}$ and $I^{+}=\{j \in \widetilde{J}: i<j \bmod T$ for every $i \in I\}$. We obtain

(a) $I \subseteq J$ and there exists $X \in \mathcal{G}$ allai $(\sigma[\widetilde{J}])$ such that $|X| \geq 2$;

(b) if $\left|I^{-}\right| \geq 2$, then $T[\widetilde{J}]$ admits a largest element $j^{+}$, and we have $I^{+}=\left\{j^{+}\right\}$and $\widetilde{J} \backslash J=\left\{j^{+}\right\}$

(c) if $\left|I^{+}\right| \geq 2$, then $T[\widetilde{J}]$ admits a smallest element $j^{-}$, and we have $I^{-}=\left\{j^{-}\right\}$and $\widetilde{J} \backslash J=\left\{j^{-}\right\}$

(d) for each $j \in \widetilde{J} \backslash J, j$ is the smallest or the largest element of $T[\widetilde{J}]$.

Proof. Let $J$ be an inclusive clan of $\sigma$ which is not a strong clan of $\sigma$. By Lemma 2.10, $\widetilde{J}$ is a strong clan of $\sigma$, which is not a limit of $\sigma$, and there is a nontrivial clan $\mathscr{J}$ of $\mathcal{Q} \operatorname{uotient}_{\sigma}(\widetilde{J})$ such that $J=\bigcup_{X \in \mathscr{J}} X$. Let $X \in \mathcal{G}$ allai $(\sigma[\widetilde{J}])$ such that $|X| \geq 2$. Since $X$ is a strong clan of $\sigma$, we have $J \cap X \neq \emptyset$ and hence $X \in \mathscr{J}$. Therefore $\{X \in \mathcal{G}$ allai $(\sigma[\widetilde{J}]):|X| \geq 2\} \subseteq \mathscr{J}$. For convenience, set

$$
\widetilde{J}_{1}=\{u \in \widetilde{J}:\{u\} \in \mathcal{G} \text { allai }(\sigma[\widetilde{J}])\}
$$


and

$$
\widetilde{J}_{\geq 2}=\bigcup_{X \in \mathcal{G} \text { allai }(\sigma[\widetilde{J}]),|X| \geq 2} X .
$$

Thus

$$
\widetilde{J} \backslash \widetilde{J}_{\geq 2}=\widetilde{J}_{1}
$$

Since $\{X \in \mathcal{G}$ allai $(\sigma[\widetilde{J}]):|X| \geq 2\} \subseteq \mathscr{J}$, we get $\widetilde{J}_{\geq 2} \subseteq J$. Moreover, since $J$ is not a strong clan of $\sigma$, we obtain

$$
\widetilde{J}_{\geq 2} \subseteq J \subsetneq \widetilde{J}
$$

Since $\mathscr{J}$ is a nontrivial clan of $\mathcal{Q}$ uotient $_{\sigma}(\widetilde{J}), \mathcal{Q u o t i e n t}_{\sigma}(\widetilde{J})$ is complete or linear. We distinguish the following two cases.

First, suppose that $\mathcal{Q u o t i e n t}_{\sigma}(\widetilde{J})$ is complete. For a contradiction, suppose that $\left|\widetilde{J} \backslash \widetilde{J}_{\geq 2}\right| \geq 2$. By (6.3), $\left|\widetilde{J}_{1}\right| \geq 2$. It follows from Proposition 3.3 that $\widetilde{J}_{1} \in \mathcal{C}$ omplete $(\sigma)$. It follows from (6.3) and (6.4) that

$$
\widetilde{J}_{1} \not J
$$

which contradicts the fact that $J$ is an inclusive clan of $\sigma$. Consequently $\left|\widetilde{J} \backslash \widetilde{J}_{\geq 2}\right| \leq 1$. By $(6.4),\left|\widetilde{J} \backslash \widetilde{J}_{\geq 2}\right|=1$. Hence $\left|\widetilde{J}_{1}\right|=1$ (i.e. Assertion 1.(a) holds) by (6.3). Denote by $j$ the unique element of $\widetilde{J}_{1}$. It follows from (6.4) that $J=\widetilde{J} \backslash\{j\}$ and $\widetilde{J}_{\geq 2}=J$ (i.e. Assertion 1.(b) holds).

Second, suppose that $\mathcal{Q u o t i e n t}_{\sigma}(\widetilde{J})$ is linear. For a contradiction, suppose that $|X|=1$ for every $X$ of $\mathcal{G}$ allai $(\sigma[\widetilde{J}])$. By Proposition $3.3, \widetilde{J} \in$ $\mathcal{L}$ inear $(\sigma)$. It follows from $(6.4)$ that $\widetilde{J} \nsubseteq J$, which contradicts the fact that $J$ is an inclusive clan of $\sigma$. Thus there exists $X \in \mathcal{G}$ allai $(\sigma[\widetilde{J}])$ such that $|X| \geq 2$. Since $X \subseteq \widetilde{J}_{\geq 2}$ and $\widetilde{J}_{\geq 2} \subseteq J$ by (6.4), it follows from the second assertion of Lemma 4.7 that $J$ is an interval of $T[\widetilde{J}]$. By minimality of $I$, we have $I \subseteq J$. Hence Assertion 2.(a) holds.

Suppose that $\left|I^{-}\right| \geq 2$. Denote by $\mathcal{K}$ the family of nonempty intervals $K$ of $T[\widetilde{J}]$ such that $\{k\} \in \mathcal{G}$ allai $(\sigma[\widetilde{J}])$ for each $k \in K$. Clearly $I^{-} \in \mathcal{K}$. Since $I$ is the smallest interval of $T[\widetilde{J}]$ containing $\widetilde{J}_{\geq 2}, I^{-}$is a maximal element of $\mathcal{K}$. Furthermore, it follows from the second assertion of Lemma 4.7 that all the elements of $\mathcal{K}$ are clans of $\sigma[\widetilde{J}]$. Therefore, it follows from the second assertion of Proposition 3.3 that $I^{-} \in \mathcal{L}$ inear $(\sigma)$. Since $J$ is an inclusive clan 
of $\sigma, I^{-} \subseteq J$. Consequently,

$$
\left\{\begin{array}{l}
\left|I^{-}\right| \geq 2 \Longrightarrow I^{-} \subseteq J \\
\text { and similarly } \\
\left|I^{+}\right| \geq 2 \Longrightarrow I^{+} \subseteq J
\end{array}\right.
$$

Suppose again that $\left|\mathcal{I}^{-}\right| \geq 2$. By (6.5), $I^{-} \subseteq J$. Since $I \subseteq J$ and $J \subsetneq \widetilde{J}$, we get $I^{+} \nsubseteq J$. It follows from (6.5) that $\left|I^{+}\right|=1$. Denote by $j^{+}$the unique element of $I^{+}$. Clearly $j^{+}$is the largest element of $T[\widetilde{J}]$, and we have $\widetilde{J} \backslash J=\left\{j^{+}\right\}$. Hence Assertion 2.(b) holds. Similarly, Assertion 2.(c) holds.

Lastly, consider $j \in \widetilde{J} \backslash J$. Since $I \subseteq J, j \in I^{-} \cup I^{+}$. For instance, assume that $j \in I^{-}$. It follows from Assertion 2.(b) that $\left|I^{-}\right| \leq 1$, so $I^{-}=\{j\}$. Clearly, $j$ is the smallest element of $T[\widetilde{J}]$. In the same manner, if $j \in I^{+}$, then $j$ is the largest element of $T[\widetilde{J}]$. Consequently, Assertion 2.(d) holds.

The next result follows from Proposition 6.6. It is used in the proof of Theorem 7.2.

Theorem 6.7. Let $\sigma$ be a 2-structure. Consider a traverse $T$ of $\sigma$. Given inclusive clans $J$ and $K$ of $\sigma$, if $J \backslash K \neq \emptyset$ and $K \backslash J \neq \emptyset$, then the following assertions hold

1. $\widetilde{J}=\widetilde{K}, \widetilde{J}$ is not a limit of $\sigma$, and $\mathcal{Q u o t i e n t}_{\sigma}(\widetilde{J})$ is linear;

2. $T[\widetilde{J}]$ admits a smallest element $j^{-}$and a largest element $j^{+}$;

3. $\left\{j^{-}\right\},\left\{j^{+}\right\} \in \mathcal{G}$ allai $(\sigma[\widetilde{J}])$, and there exists $X \in \mathcal{G}$ allai $(\sigma[\widetilde{J}])$ such that $|X| \geq 2$;

4. $\{J, K\}=\left\{\widetilde{J} \backslash\left\{j^{-}\right\}, \widetilde{J} \backslash\left\{j^{+}\right\}\right\}$, and $\widetilde{J} \backslash\left\{j^{-}, j^{+}\right\}$is the smallest interval of $T[\widetilde{J}]$ containing all the elements $Y \in \mathcal{G}$ allai $(\sigma[\widetilde{J}])$ such that $|Y| \geq 2$;

5. for each inclusive clan $H$ of $\sigma$ such that $H \neq J$ and $H \neq K$, we have $H \subseteq J \cap K$ or $J \cup K \subseteq H$.

Proof. By Proposition 6.5, $J \cap K$ is an inclusive clan of $\sigma$. Hence $J \cap K \neq \emptyset$. Therefore $J$ and $K$ are not strong clans of $\sigma$, and we can apply Proposition 6.6 to $J$ and $K$ as follows.

Since $J \cap K \neq \emptyset$, we have $\widetilde{J} \cap \widetilde{K} \neq \emptyset$ and hence $\widetilde{J} \subseteq \widetilde{K}$ or $\widetilde{K} \subseteq \widetilde{J}$. Assume that $\widetilde{J} \subseteq \widetilde{K}$. For a contradiction, suppose that $\widetilde{J} \subsetneq \widetilde{K}$. Since $K$ is not a strong clan of $\sigma, \widetilde{K}$ is not a limit of $\sigma$ by Lemma 2.10. By maximality of the elements of $\mathcal{G}$ allai $(\sigma[\widetilde{K}])$, there exists $X \in \mathcal{G}$ allai $(\sigma[\widetilde{K}])$ such that $X \supseteq \widetilde{J}$. Since $J$ is not a strong clan of $\sigma$, we have $|J| \geq 2$ and hence $|X| \geq 2$. It follows from Proposition 6.6 that $X \subseteq K$. We obtain $J \subseteq \widetilde{J} \subseteq X \subseteq K$, which contradicts $J \backslash K \neq \emptyset$. Therefore $\widetilde{\widetilde{J}}=\widetilde{K}$. 
Since $J$ is not a strong clan of $\sigma, \widetilde{J}$ is not a limit of $\sigma$ by Lemma 2.10. Suppose for a contradiction that $\mathcal{Q u o t i e n t}_{\sigma}(\widetilde{J})$ is complete. By Assertion 1.(a) of Proposition 6.6, there is a unique $j \in \widetilde{J}$ such that $\{j\} \in \mathcal{G}$ allai $(\sigma[\widetilde{J}])$. Furthermore, it follows from Assertion 1.(b) of Proposition 6.6 that $J=K=$ $\widetilde{J} \backslash\{j\}$. Thus $\mathcal{Q}$ uotient $_{\sigma}(\widetilde{J})$ is linear. Therefore the first assertion holds.

We have $\widetilde{J} \backslash J \neq \emptyset, \widetilde{J} \backslash K \neq \emptyset$ and $\widetilde{J} \backslash J \neq \widetilde{J} \backslash K$. Therefore, it follows from Assertion 2.(d) of Proposition 6.6 applied to $J$ and to $K$ that $T[\widetilde{J}]$ admits a smallest element $j^{-}$and a largest element $j^{+}$. Hence the second assertion holds. Moreover, it follows from Assertion 2.(d) of Proposition 6.6 applied to $J$ that $\widetilde{J} \backslash J \subseteq\left\{j^{-}, j^{+}\right\}$. Similarly, $\widetilde{J} \backslash K \subseteq\left\{j^{-}, j^{+}\right\}$. Since $\widetilde{J} \backslash J \neq \emptyset$, $\widetilde{J} \backslash K \neq \emptyset$ and $\widetilde{J} \backslash J \neq \widetilde{J} \backslash K$, we obtain

$$
\widetilde{J} \backslash(J \cap K)=\left\{j^{-}, j^{+}\right\} .
$$

As in Proposition 6.6, we denote by $I$ the smallest interval of $T[\widetilde{J}]$ containing all the elements $X$ of $\mathcal{G}$ allai $(\sigma[\widetilde{J}])$ such that $|X| \geq 2$. It follows from Assertion 2.(a) of Proposition 6.6 applied to $J$ and $K$ that

$$
I \subseteq J \cap K
$$

and there exists $X \in \mathcal{G}$ allai $(\sigma[\widetilde{J}])$ such that $|X| \geq 2$. Thus, it follows from (6.7) and (6.6) that $\left\{j^{-}\right\},\left\{j^{+}\right\} \in \mathcal{G}$ allai $(\sigma[\widetilde{J}])$. Hence the third assertion holds.

Since $J \cap K \subsetneq J \subsetneq \widetilde{J}$, it follows from (6.6) that $J=\widetilde{J} \backslash\left\{j^{-}\right\}$or $\widetilde{J} \backslash\left\{j^{+}\right\}$. Similarly, $K=\widetilde{J} \backslash\left\{j^{-}\right\}$or $\widetilde{J} \backslash\left\{j^{+}\right\}$. Hence we can assume that $J=\widetilde{J} \backslash\left\{j^{+}\right\}$ and $K=\widetilde{J} \backslash\left\{j^{-}\right\}$. As in Proposition 6.6, we consider $I^{-}=\{j \in \widetilde{J}: j<i$ $\bmod T$ for every $i \in I\}$ and $I^{+}=\{j \in \widetilde{J}: i<j \bmod T$ for every $i \in I\}$. Since $J=\widetilde{J} \backslash\left\{j^{+}\right\}$, it follows from Assertion 2.(c) of Proposition 6.6 applied to $J$ that $\left|I^{+}\right| \leq 1$. Furthermore, since $j^{+} \in \widetilde{J} \backslash J$, it follows from (6.7) that $j^{+} \in \widetilde{J} \backslash I$. Therefore, $i^{+} \in I^{+}$, and hence $I^{+}=\left\{j^{+}\right\}$. Similarly, $I^{-}=\left\{j^{-}\right\}$. Consequently, we obtain $I=\widetilde{J} \backslash\left\{j^{-}, j^{+}\right\}$. Hence the fourth assertion holds. Since $J=\widetilde{J} \backslash\left\{j^{+}\right\}, K=\widetilde{J} \backslash\left\{j^{-}\right\}$and $I=\widetilde{J} \backslash\left\{j^{-}, j^{+}\right\}$, we have

$$
I=J \cap K .
$$

Lastly, consider an inclusive clan $H$ of $\sigma$ such that $H \neq J$ and $H \neq K$. Since $|J| \geq 2$, it follows from Lemma 6.4 that $H \cap J \neq \emptyset$. Thus $H \cap \widetilde{J} \neq \emptyset$, and hence $H \subsetneq \widetilde{J}$ or $\widetilde{J} \subseteq H$. If $\widetilde{J} \subseteq H$, then $J \cup K \subseteq H$ because $\widetilde{J}=\widetilde{K}$. Therefore suppose that

$$
H \subsetneq \widetilde{J} .
$$


We show that $H \subseteq J \cap K$. Set

$$
\mathscr{H}=\{X \in \mathcal{G} \text { allai }(\sigma[\widetilde{J}]): X \cap H \neq \emptyset\} .
$$

Since $H$ is an inclusive clan of $\sigma, H \cap X \neq \emptyset$ for every $X \in \mathcal{G}$ allai $(\sigma[\widetilde{J}])$ such that $|X| \geq 2$. Therefore

$$
\{X \in \mathcal{G} \text { allai }(\sigma[\widetilde{J}]):|X| \geq 2\} \subseteq \mathscr{H} .
$$

Recall that there exists $X \in \mathcal{G}$ allai $(\sigma[\widetilde{J}])$ such that $|X| \geq 2$ (see the third assertion). Thus $\mathscr{H} \neq \emptyset$, and we distinguish the following two cases.

1. Suppose that $|\mathscr{H}|=1$. Denote by $Y$ the unique element of $\mathscr{H}$. We have $H \subseteq Y$. Since $\{X \in \mathcal{G}$ allai $(\sigma[\widetilde{J}]):|X| \geq 2\} \neq \emptyset$ (see the third assertion), it follows from (6.10) that $Y$ is the unique element of $\mathcal{G}$ allai $(\sigma[\widetilde{J}])$ such that $|Y| \geq 2$. Furthermore, $Y$ is an interval of $T[\widetilde{J}]$ by Assertion A1 (see Definition 4.1). It follows from the definition of $I$ that $I=Y$. Since $H \subseteq Y$, we get $H \subseteq I$. It follows from (6.8) that $H \subseteq J \cap K$.

2. Suppose that $|\mathscr{H}| \geq 2$. We obtain

$$
H=\bigcup_{X \in \mathscr{H}} X
$$

It follows from the second assertion of Lemma 4.7 that $H$ is an interval of $T[\widetilde{J}]$. By $(6.10),\{X \in \mathcal{G}$ allai $(\sigma[\widetilde{J}]):|X| \geq 2\} \subseteq \mathscr{H}$. It follows from the definition of $I$ that $I \subseteq H$. Since $I=J \cap K$ by (6.8), we get $J \cap K \subseteq H$. Recall that $\widetilde{J}=\left\{j^{-}\right\} \cup(J \cap K) \cup\left\{j^{+}\right\}, J=\left\{j^{-}\right\} \cup(J \cap K)$ and $K=(J \cap K) \cup\left\{j^{+}\right\}$. Moreover, recall that $H \subsetneq \widetilde{J}$ by (6.9). Since $H \neq J$ and $H \neq K$, we obtain $H=J \cap K$.

The next result is an immediate consequence of Theorem 6.7. It is used in the proof of Proposition 7.7.

Corollary 6.8. If $\sigma$ is a symmetric 2-structure, then the set of inclusive clans of $\sigma$ endowed with inclusion is a linear order.

We complete the section with a result on primitive bounds of asymmetric 2 -structures. It is used in the proof of Theorem 7.1.

Theorem 6.9. Let $\sigma$ be an asymmetric 2-structure. If $\sigma$ admits an inclusive clan $J$ such that $J \neq V(\sigma)$, then $p(\sigma)=1$.

Proof. Since $\sigma$ admits an inclusive clan $J$ such that $J \neq V(\sigma)$, we have $|V(\sigma)| \geq 3$. It follows from Lemma 6.3 and (6.1) that $\sigma$ admits an inclusive 
clan $J$ which is a nontrivial clan of $\sigma$. In particular, $\sigma$ is imprimitive, and hence

$$
p(\sigma) \geq 1 .
$$

By Proposition 4.2, $\sigma$ admits a traverse $T$. Furthermore $T$ admits a dense bicoloring $\beta$ by Proposition 4.12. Given $e \in E(\sigma)$, set $e_{0}=e$ and $e_{1}=e^{\star}$. Let $a \notin V(\sigma)$. We associate with $\beta$ the faithful extension $\tau_{\beta}$ of $\sigma$ defined on $V(\sigma) \cup\{a\}$ satisfying

$$
\begin{gathered}
(x, a)_{\tau_{\beta}}=e_{\beta(x)} \text { for every } x \in J ; \\
(x, a)_{\tau_{\beta}}=\left((x, J)_{\sigma}\right)^{\star} \text { for every } x \in V(\sigma) \backslash J .
\end{gathered}
$$

If $\tau_{\beta}$ is imprimitive, then we establish the following assertions

- $T$ has a smallest element $v^{-}$and a largest element $v^{+}$;

- there exists $v \in\left\{v^{-}, v^{+}\right\}$such that $J=V(\sigma) \backslash\{v\}$;

- by denoting by $u$ the unique element of $\left\{v^{-}, v^{+}\right\} \backslash\{v\}$, we have

$$
e_{\beta(u)}=(u, v)_{\sigma} .
$$

Indeed, suppose that $\tau_{\beta}$ is imprimitive, and consider a nontrivial clan $D_{\beta}$ of $\tau_{\beta}$. To prove the three assertions above, we need some preliminary facts (see (6.14), (6.15), (6.16) and (6.17)).

Suppose for a contradiction that $a \notin D_{\beta}$. We obtain that $D_{\beta}$ is a clan of $\sigma$ such that $\left|D_{\beta}\right| \geq 2$. Hence $J \cap D_{\beta}$ is a clan of $\sigma$. By Corollary 4.9, $J \cap D_{\beta}$ is an interval of $T$. For $x, y \in J \cap D_{\beta}$, we have $(x, a)_{\tau_{\beta}}=(y, a)_{\tau_{\beta}}$. It follows from (6.12a) that $e_{\beta(x)}=e_{\beta(y)}$ and hence $\beta(x)=\beta(y)$. Therefore $\beta_{\left\lceil J \cap D_{\beta}\right.}$ is constant. Since $D_{\beta}$ is a clan of $\sigma$ such that $\left|D_{\beta}\right| \geq 2$, it follows from Lemma 6.4 that $J \cap D_{\beta} \neq \emptyset$. Since $\beta$ is a dense bicoloring of $T$, we obtain $\left|J \cap D_{\beta}\right|=1$. Denote by $z$ the unique element of $J \cap D_{\beta}$. We show that $D_{\beta} \backslash\{z\}$ is a clan of $\sigma$. It suffices to verify that $D_{\beta} \backslash\{z\}$ is a clan of $\sigma\left[D_{\beta}\right]$, that is, $z \longleftrightarrow D_{\beta} \backslash\{z\}$. Let $x, y \in D_{\beta} \backslash\{z\}$. Since $D_{\beta}$ is a clan of $\tau_{\beta}$, we have $(x, a)_{\tau_{\beta}}=(y, a)_{\tau_{\beta}}$. It follows from $(6.12 \mathrm{~b})$ that $(x, z)_{\sigma}=(y, z)_{\sigma}$. Consequently $D_{\beta} \backslash\{z\}$ is a clan of $\sigma$. It follows from Lemma 6.4 that $\left|D_{\beta} \backslash\{z\}\right|=1$. Hence $\left|D_{\beta}\right|=2$ and $\sigma\left[D_{\beta}\right]$ is linear. By Lemma 3.2, we obtain that $D_{\beta} \subseteq L(\sigma)$. Since $D_{\beta} \backslash J \neq \emptyset, J$ is not an inclusive clan of $\sigma$. It follows that

$$
a \in D_{\beta} .
$$


We get $D_{\beta} \backslash\{a\}$ is a clan of $\sigma$. Suppose for a contradiction that $J \cap\left(D_{\beta} \backslash\right.$ $\{a\})=\emptyset$. We have $\left(D_{\beta} \backslash\{a\}\right) \longleftrightarrow{ }_{\sigma} J$. It follows that $a \longleftrightarrow_{\tau_{\beta}} J$. By (6.12a), $\beta_{\uparrow J}$ is constant. Since $J$ is an interval of $T$ by Corollary $4.9, \beta$ is not a dense bicoloring of $T$. Consequently, $J \cap\left(D_{\beta} \backslash\{a\}\right) \neq \emptyset$. Therefore $J \cup D_{\beta}$ is a clan of $\tau_{\beta}$. It follows from $(6.12 \mathrm{~b})$ that

$$
J \cup\left(D_{\beta} \backslash\{a\}\right)=V(\sigma) .
$$

Since $J \neq V(\sigma)$, it follows from (6.15) that

$$
\left(D_{\beta} \backslash\{a\}\right) \backslash J \neq \emptyset .
$$

Furthermore, since $D_{\beta}$ is a nontrivial clan of $\tau_{\beta}$, it follows from (6.14) and (6.15) that

$$
J \backslash\left(D_{\beta} \backslash\{a\}\right) \neq \emptyset .
$$

Thus $\left(D_{\beta} \backslash\{a\}\right) \backslash J$ is a clan of $\sigma$. It follows from Lemma 6.4 that $\mid\left(D_{\beta} \backslash\right.$ $\{a\}) \backslash J \mid \leq 1$. Hence $\left|\left(D_{\beta} \backslash\{a\}\right) \backslash J\right|=1$ by (6.16). Denote by $v$ the unique element of $\left(D_{\beta} \backslash\{a\}\right) \backslash J$. It follows from (6.15) that

$$
J=V(\sigma) \backslash\{v\} .
$$

By Corollary 4.9, $J$ is an interval of $T$. Since $J=V(\sigma) \backslash\{v\}, v$ is the smallest or the largest element of $T$.

By (6.16), $J \backslash\left(D_{\beta} \backslash\{a\}\right)$ is a clan of $\sigma$. Since $D_{\beta} \backslash\{a\}$ is a clan of $\sigma$, we have $\left(D_{\beta} \backslash\{a\}\right) \longleftrightarrow_{\sigma} J \backslash\left(D_{\beta} \backslash\{a\}\right)$. Since $D_{\beta}$ is a clan of $\tau_{\beta}$, we obtain

$$
a \longleftrightarrow \tau_{\beta} J \backslash\left(D_{\beta} \backslash\{a\}\right) .
$$

By (6.12a), $\beta_{\left\lceil J \backslash\left(D_{\beta} \backslash\{a\}\right)\right.}$ is constant. Moreover, $J \backslash\left(D_{\beta} \backslash\{a\}\right)$ is an interval of $T$ by Corollary 4.9. Since $\beta$ is a dense bicoloring of $T,\left|J \backslash\left(D_{\beta} \backslash\{a\}\right)\right| \leq 1$. Thus $\left|J \backslash\left(D_{\beta} \backslash\{a\}\right)\right|=1$ by (6.17). Denote by $u$ the unique element of $J \backslash\left(D_{\beta} \backslash\{a\}\right)$. It follows from (6.14) and (6.15) that

$$
D_{\beta}=V\left(\tau_{\beta}\right) \backslash\{u\} .
$$

It follows that $V(\sigma) \backslash\{u\}$ is a clan of $\sigma$. By Corollary 4.9, $V(\sigma) \backslash\{u\}$ is an interval of $T$. Therefore $u$ is the smallest or the largest element of $T$. Since $u \in J$, we have $u \neq v$. Consequently, $T$ has a smallest element $v^{-}$and a largest element $v^{+}$. Hence $v \in\left\{v^{-}, v^{+}\right\}$and $\{u\}=\left\{v^{-}, v^{+}\right\} \backslash\{v\}$. Since $D_{\beta}$ is a clan of $\tau_{\beta},(u, a)_{\tau_{\beta}}=(u, v)_{\sigma}$. Thus (6.13) follows from (6.12a). 
We conclude as follows. Clearly (6.13) cannot hold for both dense bicolorings $\beta$ and $1-\beta$ of $T$. Consequently $\tau_{\beta}$ or $\tau_{1-\beta}$ is primitive. Thus $p(\sigma) \leq 1$. By $(6.11), p(\sigma)=1$.

\section{Primitive bounds of reversible 2-structures}

Let $\sigma$ be a reversible 2 -structure such that $|V(\sigma)| \geq 2$. Suppose that $|E(\sigma)|=$ 1 , that is, $\sigma$ is complete. We consider extensions of $\sigma$ which are identifiable with graphs. When $|V(\sigma)|<\aleph_{0}$, it follows from [27, Theorem 2.45] that there exists a primitive extension $\tau$ of $\sigma$ such that $\tau$ is symmetric, $|E(\tau)|=2$ and $|V(\tau) \backslash V(\sigma)| \geq\left\lceil\log _{2}(|V(\sigma)|+1)\right\rceil$. This result is easily adaptable when $|V(\sigma)| \geq \aleph_{0}$ by replacing $\left\lceil\log _{2}(|V(\sigma)|+1)\right\rceil$ by $\log _{2}(|V(\sigma)|)$.

Now, we consider reversible 2 -structures $\sigma$ such that $|E(\sigma)| \geq 2$. We begin with tournaments, that is, asymmetric 2-structures $\sigma$ such that $|E(\sigma)|=$ 2. As mentioned in Section 1, the next theorem was proved by Erdös et al. [11].

Theorem 7.1. Given a 2-structure $\sigma$ such that $\sigma$ is asymmetric and $|E(\sigma)|=$ 2 , we have $p(\sigma) \leq 2$. Moreover, $p(\sigma)=2$ if and only if $\sigma$ is a finite linear order such that $|V(\sigma)|$ is odd.

Proof. By Corollary 4.14, it suffices to prove that if $p(\sigma) \geq 2$, then $\sigma$ is a linear order. Suppose that $p(\sigma) \geq 2$. Denote by $\mathcal{L}_{\text {odd }}(\sigma)$ the family of $L \in \mathcal{L}$ inear $(\sigma)$ such that $|L|<\aleph_{0}$ and $|L|$ is odd, and denote by $\mathcal{P}_{3}(\sigma)$ the family of $P \in \mathcal{P}$ rimitive $(\sigma)$ such that $|P|=3$.

Let $a \notin V(\sigma)$. Given $P \in \mathcal{P}$ rimitive $(\sigma) \backslash \mathcal{P}_{3}(\sigma)$, it follows from Lemma 5.5 that $\sigma$ admits a primitive and faithful extension $\tau_{P}$ defined on $V(\sigma) \cup\{a\}$ such that $\tau_{P}[P \cup\{a\}]$ is primitive. Given $L \in \mathcal{L} \operatorname{inear}(\sigma) \backslash \mathcal{L}_{\text {odd }}(\sigma)$, it follows from Corollary 4.14 that $\sigma$ admits a primitive and faithful extension $\tau_{L}$ defined on $V(\sigma) \cup\{a\}$ such that $\tau_{L}[L \cup\{a\}]$ is primitive.

By Proposition 4.2, $\sigma$ admits a traverse $T$. Furthermore $T$ admits a dense bicoloring $\beta$ by Proposition 4.12. Set $E(\sigma)=\left\{e_{0}, e_{1}\right\}$ and $\mathscr{F}=(\mathcal{L}$ inear $(\sigma) \backslash$ $\left.\mathcal{L}_{\text {odd }}(\sigma)\right) \cup\left(\mathcal{P}\right.$ rimitive $\left.(\sigma) \backslash \mathcal{P}_{3}(\sigma)\right)$. By Theorem 3.7, the elements of $\mathscr{F}$ are pairwise disjoint. Thus, we can consider the faithful extension $\tau$ of $\sigma$ defined on $V(\sigma) \cup\{a\}$ satisfying

- for each $X \in \mathscr{F}, \tau[X \cup\{a\}]=\tau_{X}\left[C_{\cup}\{a\}\right]$;

- for each $v \in V(\sigma) \backslash\left(\bigcup_{X \in \mathscr{F}} X\right),(v, a)_{\tau}=e_{\beta(v)}$.

Since $p(\sigma) \geq 2, \tau$ admits a nontrivial clan $D$. It follows from Corollary 5.8 
that

$$
\left\{\begin{array}{l}
a \in D, \\
\text { for every clan } C \text { of } \sigma \text { such that }|C| \geq 2, C \cap(D \backslash\{a\}) \neq \emptyset, \\
\text { and } \\
\bigcup_{X \in \mathscr{F}} X \subseteq D \backslash\{a\} .
\end{array}\right.
$$

Since $p(\sigma) \geq 2, D \backslash\{a\}$ is not an inclusive clan of $\sigma$ by Theorem 6.9. It follows that

$$
\left(\bigcup_{X \in \mathcal{L}_{\text {odd }}(\sigma) \cup \mathcal{P}_{3}(\sigma)} X\right) \nsubseteq D \backslash\{a\} .
$$

We show that

$$
\left(\bigcup_{P \in \mathcal{P}_{3}(\sigma)} P\right) \subseteq D \backslash\{a\} .
$$

For a contradiction, suppose that (7.1) does not hold, and consider $P \in$ $\mathcal{P}_{3}(\sigma)$ such that $P \nsubseteq D \backslash\{a\}$. By Corollary 5.8, $P \cap(D \backslash\{a\}) \neq \emptyset$. Moreover $P$ is a strong module of $\sigma$ because $\sigma[P]$ is primitive. Since $P \nsubseteq D \backslash\{a\}$, we get $D \backslash\{a\} \subsetneq P$. Since $\sigma[P]$ is primitive, we obtain $|D \backslash\{a\}|=1$. Since $\bigcup_{X \in \mathscr{F}} X \subseteq D \backslash\{a\}$ by Corollary 5.8, we obtain $\mathscr{F}=\emptyset$. Furthermore, consider $C \in \mathcal{L}_{\text {odd }}(\sigma) \cup \mathcal{P}_{3}(\sigma)$. By Corollary 5.8, $C \cap(D \backslash\{a\}) \neq \emptyset$. Therefore $C \cap P \neq \emptyset$ and it follows from Theorem 3.7 that $C=P$. Consequently,

$$
\mathcal{L} \text { inear }(\sigma) \cup \mathcal{P} \text { rimitive }(\sigma)=\{P\}
$$

By Corollary 5.8, $X \cap(D \backslash\{a\}) \neq \emptyset$ for every strong clan $X$ of $\sigma$ such that $|X| \geq 2$. We obtain that $P$ is an inclusive clan of $\sigma$. Since $p(\sigma) \geq 2$, it follows from Theorem 6.9 that $P=V(\sigma)$. Thus $\sigma$ is primitive, which implies $p(\sigma)=0$. It follows that (7.1) holds. Consequently, there exists $L \in \mathcal{L}_{\text {odd }}(\sigma)$ such that $L \nsubseteq D \backslash\{a\}$. By Corollary 5.8, $L \cap(D \backslash\{a\}) \neq \emptyset$. Hence $\widetilde{L} \cap(D \backslash\{a\}) \neq \emptyset$ and necessarily $D \backslash\{a\} \subseteq \widetilde{L}$. Lastly, consider $L^{\prime} \in \mathcal{L}_{\text {odd }}(\sigma) \backslash\{L\}$. By Corollary 5.8, $L^{\prime} \cap(D \backslash\{a\}) \neq \emptyset$ and hence $L^{\prime} \cap \widetilde{L} \neq \emptyset$. If $\widetilde{L} \subseteq L^{\prime}$, then $L \subseteq L^{\prime}$, and we have $L=L^{\prime}$ by Theorem 3.7. Therefore $L^{\prime} \subseteq \widetilde{\widetilde{L}}$. It follows that

$$
\left(\bigcup_{L^{\prime} \in \mathcal{L}_{\text {odd }}(\sigma)} L^{\prime}\right) \subseteq \widetilde{L} .
$$

We obtain that $\widetilde{L}$ is an inclusive clan of $\sigma$. Since $p(\sigma) \geq 2$, it follows from Theorem 6.9 that $\widetilde{L}=V(\sigma)$. By Proposition 3.3, $V(\sigma)$ is not a limit of $\sigma$, 
and $\mathcal{Q u o t i e n t}_{\sigma}(V(\sigma))$ is linear. Since $p(\sigma) \geq 2$, it follows from Theorem 4.16 that $|X|=1$ for each $X \in \mathcal{G}$ allai $(\sigma)$. In other words, $\sigma$ is a linear order.

We continue with reversible 2 -structures $\sigma$ such that $|E(\sigma)| \geq 3$ or $\sigma$ is symmetric and $|E(\sigma)|=2$.

Theorem 7.2. Let $\sigma$ be a reversible 2-structure.

1. Suppose that $c(\sigma) \geq 2$. If $c(\sigma)<|E(\sigma)|$ or if $c(\sigma)=|E(\sigma)|$ and $|E(\sigma)| \geq \aleph_{0}$, then $p(\sigma)=\mathfrak{l o g}_{|E(\sigma)|}(c(\sigma))=1$.

2. Suppose that $c(\sigma)=1$. If $|E(\sigma)| \geq 3$ or if $\sigma$ is symmetric and $|E(\sigma)|=$ 2 , then $p(\sigma) \leq 1$.

Proof. Let $a \notin V(\sigma)$. We construct a primitive and faithful extension of $\sigma$ to $V(\sigma) \cup\{a\}$.

First, suppose that $\mathcal{C}$ omplete $(\sigma) \neq \emptyset$. Consider any $C \in \mathcal{C}$ omplete $(\sigma)$. There exists $e \in E(\sigma)$ such that for distinct $c, d \in C$, we have $(c, d)_{\sigma}=$ $(d, c)_{\sigma}=e$. Since $\mathcal{C}$ omplete $(\sigma) \neq \emptyset, c(\sigma) \geq 2$. Thus $|E(\sigma)|>c(\sigma)$ or $|E(\sigma)|=$ $c(\sigma)$ and $|E(\sigma)| \geq \aleph_{0}$, Since $c(\sigma) \geq|C|$, there exists an injection $A_{C}: C \longrightarrow$ $E(\sigma) \backslash\{e\}$. We consider a faithful extension $\tau_{C}$ of $\sigma$ defined on $V(\sigma) \cup\{a\}$ satisfying $\overrightarrow{\tau_{C}}(a)_{\lceil C}=A_{C}$. It is easy to verify that $\tau_{C}[C \cup\{a\}]$ is primitive.

Second, suppose that $\mathcal{L}$ inear $(\sigma) \neq \emptyset$. Consider any $L \in \mathcal{L}$ inear $(\sigma)$. We do not have $\sigma$ is symmetric and $|E(\sigma)|=2$. Therefore $|E(\sigma)| \geq 3$. By Corollary 4.15, there exists a faithful extension $\tau_{L}$ of $\sigma$ defined on $V(\sigma) \cup\{a\}$ such that $\tau_{L}[L \cup\{a\}]$ is primitive.

Third, suppose that $\mathcal{P}$ rimitive $(\sigma) \neq \emptyset$. Consider any $P \in \mathcal{P}$ rimitive $(\sigma)$. By Lemma 5.5, there exists a faithful extension $\tau_{P}$ of $\sigma$ defined on $V(\sigma) \cup\{a\}$ such that $\tau_{P}[P \cup\{a\}]$ is primitive.

By Proposition 4.2, $\sigma$ admits a traverse $T$. Let $e_{0} \in E(\sigma)$. Since $|E(\sigma)| \geq$ 3 or $\sigma$ is symmetric and $|E(\sigma)|=2$, there exists

$$
e_{1} \in E(\sigma) \backslash\left\{e_{0},\left(e_{0}\right)^{\star}\right\} .
$$

By Theorem 3.7, the elements of $\mathcal{C}$ omplete $(\sigma) \cup \mathcal{L}$ inear $(\sigma) \cup \mathcal{P}$ rimitive $(\sigma)$ are pairwise disjoint. Therefore, with each dense bicoloring $\beta$ of $T$, we can associate the faithful extension $\tau_{\beta}$ of $\sigma$ defined on $V(\sigma) \cup\{a\}$ satisfying

- for every $C \in \mathcal{C}$ omplete $(\sigma) \cup \mathcal{L}$ inear $(\sigma) \cup \mathcal{P}$ rimitive $(\sigma), \tau_{\beta}[C \cup\{a\}]=$ $\tau_{C}[C \cup\{a\}]$;

- for each $v \in V(\sigma) \backslash(C(\sigma) \cup L(\sigma) \cup P(\sigma)),(v, a)_{\tau_{\beta}}=e_{\beta(v)}$.

We establish the following fact for each dense bicoloring $\beta$ of $T$. If $\tau_{\beta}$ is imprimitive, then for every nontrivial clan $C_{\beta}$ of $\tau_{\beta}$, we have $a \in C_{\beta}$ and $C_{\beta} \backslash\{a\}$ is an inclusive clan of $\sigma$. 
Indeed, suppose that $\tau_{\beta}$ is imprimitive, and consider a nontrivial clan $C_{\beta}$ of $\tau_{\beta}$. Given $C \in \mathcal{C}$ omplete $(\sigma)$, we have $\tau_{\beta}[C \cup\{a\}]=\tau_{C}[C \cup\{a\}]$ is primitive. Therefore, for each clan $D$ of $\sigma[C]$ such that $|D| \geq 2, D$ is not a clan of $\tau_{\beta}[C \cup\{a\}]$ and hence of $\tau_{\beta}$. Thus $\{D \in \mathcal{C}$ lans $(\sigma[C]):|D| \geq 2\} \cap \mathcal{C} \operatorname{lans}(\tau)=\emptyset$. It follows from Corollary 5.8 applied with $\mathscr{F}=\mathcal{L}$ inear $(\sigma) \cup \mathcal{P}$ rimitive $(\sigma)$ that

$$
\left\{\begin{array}{l}
a \in C_{\beta}, \\
\text { for every strong clan } X \text { of } \sigma \text { such that }|X| \geq 2, X \cap\left(C_{\beta} \backslash\{a\}\right) \neq \emptyset, \\
\text { and } \\
C(\sigma) \cup L(\sigma) \cup P(\sigma) \subseteq C_{\beta} \backslash\{a\} .
\end{array}\right.
$$

Therefore, $C_{\beta} \backslash\{a\}$ is an inclusive clan of $\sigma$.

We conclude as follows. By Proposition 4.12, $T$ admits a dense bicoloring $\beta$. Suppose that $\tau_{\beta}$ is imprimitive, and consider a nontrivial clan $C_{\beta}$ of $\tau_{\beta}$. We have $a \in C_{\beta}$, and $C_{\beta} \backslash\{a\}$ is an inclusive clan of $\sigma$. Since $C(\sigma) \cup L(\sigma) \cup P(\sigma) \subseteq C_{\beta} \backslash\{a\},(v, a)_{\tau_{\beta}}=e_{\beta(v)}$ for every $v \in V(\sigma) \backslash\left(C_{\beta} \backslash\{a\}\right)$. Clearly $1-\beta$ is also a dense bicoloring of $T$. Similarly, suppose that $\tau_{1-\beta}$ is imprimitive, and consider a nontrivial clan $C_{1-\beta}$ of $\tau_{1-\beta}$. We obtain $a \in C_{1-\beta}, C_{1-\beta} \backslash\{a\}$ is an inclusive clan of $\sigma$, and $(v, a)_{\tau_{1-\beta}}=e_{1-\beta(v)}$ for every $v \in V(\sigma) \backslash\left(C_{1-\beta} \backslash\{a\}\right)$. It follows that $\left(C_{\beta} \backslash\{a\}\right) \cup\left(C_{1-\beta} \backslash\{a\}\right)=V(\sigma)$. By Theorem 6.7, $\left(\widetilde{C_{\beta} \backslash\{a\}}\right)=\left(\widetilde{C_{1-\beta \backslash} \backslash a}\right)$. Hence $\left(\widetilde{C_{\beta} \backslash\{a}\right)=V(\sigma)$. Furthermore, it follows from Theorem 6.7 that

- $V(\sigma)$ is not a limit of $\sigma$, and $\mathcal{Q u o t i e n t}_{\sigma}(V(\sigma))$ is linear;

- the traverse $T$ admits a smallest element $v^{-}$and a largest element $v^{+}$;

- $\left\{v^{-}\right\},\left\{v^{+}\right\} \in \mathcal{G}$ allai $(\sigma)$;

- by interchanging $C_{\beta} \backslash\{a\}$ and $C_{1-\beta} \backslash\{a\}$, we have $C_{\beta} \backslash\{a\}=V(\sigma) \backslash$ $\left\{v^{+}\right\}$and $C_{1-\beta} \backslash\{a\}=V(\sigma) \backslash\left\{v^{-}\right\}$.

Since $C_{\beta} \backslash\{a\}=V(\sigma) \backslash\left\{v^{+}\right\}$, we get

$$
\left(v^{-}, v^{+}\right)_{\sigma}=\left(v^{-}, v^{+}\right)_{\tau_{\beta}}=\left(a, v^{+}\right)_{\tau_{\beta}}=\left(\left(v^{+}, a\right)_{\tau_{\beta}}\right)^{\star}=\left(e_{\beta\left(v^{+}\right.}\right)^{\star} .
$$

Similarly, since $C_{1-\beta} \backslash\{a\}=V(\sigma) \backslash\left\{v^{-}\right\}$, we get

$$
\left(v^{-}, v^{+}\right)_{\sigma}=\left(v^{-}, v^{+}\right)_{\tau_{1-\beta}}=\left(v^{-}, a\right)_{\tau_{1-\beta}}=e_{1-\beta\left(v^{-}\right)} .
$$

Since $\left\{v^{-}\right\},\left\{v^{+}\right\} \in \mathcal{G}$ allai $(\sigma)$ and $v^{-}<v^{+} \bmod T$, it follows from Remark 4.3 that $\left(v^{-}, v^{+}\right)_{\sigma}=e_{V(\sigma)}$. Therefore $e_{\beta\left(v^{+}\right)}=\left(e_{V(\sigma)}\right)^{\star}$ and $e_{1-\beta\left(v^{-}\right)}=$ $e_{V(\sigma)}$. Hence $\left\{e_{0}, e_{1}\right\}=\left\{e_{V(\sigma)},\left(e_{V(\sigma)}\right)^{\star}\right\}$, which contradicts (7.2). Consequently, $\tau_{\beta}$ or $\tau_{1-\beta}$ is primitive. 
The next proposition provides a natural lower bound of the primitivity bound.

Proposition 7.3. For a reversible 2-structure $\sigma$ such that $|E(\sigma)| \geq 2$, we have $p(\sigma) \geq \mathfrak{l o g}_{|E(\sigma)|}(c(\sigma))$.

Proof. Consider a set $S$ such that $S \cap V(\sigma)=\emptyset$, and

$$
|E(\sigma)|^{|S|}<c(\sigma) .
$$

Let $\tau$ be any faithful extension of $\sigma$ defined on $V(\sigma) \cup S$. It follows from (7.3) that there exists $C \in \mathcal{C}$ omplete $(\sigma)$ such that

$$
|E(\sigma)|^{|S|}<|C| .
$$

Now, consider the function

$$
\begin{aligned}
\varphi: C & \longrightarrow E(\sigma)^{S} \\
c & \longmapsto \vec{\tau}(c)_{\lceil S} .
\end{aligned}
$$

It follows from (7.4) that there exist distinct $c, d \in C$ such that $\varphi(c)=\varphi(d)$, that is,

$$
\vec{\tau}(c)_{\lceil S}=\vec{\tau}(d)_{\lceil S} .
$$

Since $\sigma[C]$ is complete, $\{c, d\}$ is a clan of $\sigma[C]$. Since $C$ is a clan of $\sigma$, we obtain that $\{c, d\}$ is a clan of $\sigma$. It follows from (7.5) that $\{c, d\}$ is a nontrivial clan of $\tau$. Thus $\tau$ is imprimitive. It follows from (7.3) that $|E(\sigma)|^{p(\sigma)} \geq c(\sigma)$. Therefore $p(\sigma) \geq \mathfrak{l o g}_{|E(\sigma)|}(c(\sigma))$.

Given Theorems 7.1 and 7.2, we consider reversible 2-structures $\sigma$ such that $|E(\sigma)| \geq 2$, and $c(\sigma)>|E(\sigma)|$ or $c(\sigma)=|E(\sigma)|$ and $|E(\sigma)|<\aleph_{0}$. The following result generalizes $[2$, Theorem 1.4] for finite graphs. In spite of the length of its proof, it is simpler and shorter than the original proof for finite graphs.

Theorem 7.4. Given a reversible 2-structure $\sigma$, if $2 \leq|E(\sigma)| \leq c(\sigma)<\aleph_{0}$, then $p(\sigma) \leq\left\lceil\log _{|E(\sigma)|}(c(\sigma)+1)\right\rceil$.

Proof. Since $c(\sigma)<\aleph_{0}$, there exists $C_{\max } \in \mathcal{C}$ omplete $(\sigma)$ such that $\left|C_{\max }\right|=$ $c(\sigma)$. Denote by $e_{\max }$ the element of $E(\sigma)$ such that for distinct $c, d \in C_{\max }$, $(c, d)_{\sigma}=e_{\max }$ and $(d, c)_{\sigma}=e_{\max }$. Also consider a set $S^{\prime}$ such that $S^{\prime} \cap V(\sigma)=$ 
$\emptyset$ and $\left|S^{\prime}\right|=\left\lceil\log _{|E(\sigma)|}(c(\sigma)+1)\right\rceil$. For convenience, for each $e \in E(\sigma)$, we consider the function

$$
\begin{aligned}
\bar{e}: & S^{\prime} \\
s^{\prime} & \longmapsto E(\sigma) \\
&
\end{aligned}
$$

By Lemma 5.4, there exists a faithful extension $\tau_{\max }$ of $\sigma$ defined on $V(\sigma) \cup S^{\prime}$ satisfying

- $\tau_{\max }\left[C_{\max } \cup S^{\prime}\right]$ is primitive;

- for every $s^{\prime} \in S^{\prime}$, there is $c \in C_{\max }$ such that

$$
\left(\overrightarrow{\tau_{\max }}(c)\right)^{-1}\left(\left\{e_{\max }\right\}\right) \cap S^{\prime}=\left\{s^{\prime}\right\} .
$$

Now consider $C \in \mathcal{C}$ omplete $(\sigma) \backslash\left\{C_{\max }\right\}$. Denote by $e_{C}$ the element of $E(\sigma)$ such that for distinct $c, d \in C,(c, d)_{\sigma}=e_{C}$ and $(d, c)_{\sigma}=e_{C}$. Since $|C| \leq c(\sigma)<|E(\sigma)|^{\left|S^{\prime}\right|}$, there is an injection

$$
\left.B_{C}: C \longrightarrow E(\sigma)^{\left|S^{\prime}\right|} \backslash\left\{\overline{e_{C}}\right\} \quad \text { (see }(7.6)\right) .
$$

There is a faithful extension $\tau_{C}$ of $\sigma$ defined on $V(\sigma) \cup S^{\prime}$ such that $\tau_{C}\left[S^{\prime}\right]=$ $\tau_{\max }\left[S^{\prime}\right]$, and $\overrightarrow{\tau_{C}}(c)_{i S^{\prime}}=B_{C}(c)$ for each $c \in C$. We construct a primitive and faithful extension of $\sigma$ to $V(\sigma) \cup S^{\prime}$ as follows. By Proposition 4.2, $\sigma$ admits a traverse $T$. Furthermore $T$ admits a dense bicoloring $\beta$ by Proposition 4.12. Since $|E(\sigma)| \geq 2$ and $\left|S^{\prime}\right| \geq 2$, there exist distinct $A_{0}, A_{1} \in E(\sigma)^{S^{\prime}} \backslash\{\bar{e}$ : $e \in E(\sigma)\}$ (see (7.6)). Recall that the elements of $\mathcal{C}$ omplete $(\sigma)$ are pairwise disjoint by Theorem 3.7. Therefore, there exists a faithful extension $\tau$ of $\sigma$ defined on $V(\sigma) \cup S^{\prime}$ satisfying

- $\tau\left[C_{\max } \cup S^{\prime}\right]=\tau_{\max }\left[C_{\max } \cup S^{\prime}\right]$;

- for each $C \in \mathcal{C}$ omplete $(\sigma) \backslash\left\{C_{\max }\right\}, \tau\left[C \cup S^{\prime}\right]=\tau_{C}\left[C \cup S^{\prime}\right]$;

- for each $v \in V(\sigma) \backslash C(\sigma), \vec{\tau}(v)_{\left\lceil S^{\prime}\right.}=A_{\beta(v)}$.

To conclude, we prove that $\tau$ is primitive. Let $D_{\tau}$ be a clan of $\tau$ such that $\left|D_{\tau}\right| \geq 2$. We have to show that $D_{\tau}=V(\tau)$. It is easy to verify that (5.1a) (see Lemma 5.7) is satisfied. Hence it follows from Lemma 5.7 applied with $\mathscr{F}=\emptyset$ that $D_{\tau} \cap S^{\prime} \neq \emptyset$. Moreover, since $\tau\left[C_{\max } \cup S^{\prime}\right]$ is primitive, $D_{\tau} \cap V(\sigma) \neq \emptyset$.

For a contradiction, suppose that $C_{\max } \cap D_{\tau}=\emptyset$. Since $\tau\left[C_{\max } \cup S^{\prime}\right]$ is primitive, there is $s^{\prime} \in S^{\prime}$ such that $\left(C_{\max } \cup S^{\prime}\right) \cap D_{\tau}=\left\{s^{\prime}\right\}$. Consider $v \in D_{\tau} \cap V(\sigma)$. Since $C_{\max }$ is a clan of $\sigma$, there is $e \in E(\sigma)$ such that $\left(v, C_{\max }\right)_{\sigma}=e$. Since $D_{\tau}$ is a clan of $\tau$, we get $\left(s^{\prime}, C_{\max }\right)_{\tau}=e$. It follows 
that $e^{\star}=\vec{\tau}(c)\left(s^{\prime}\right)=\overrightarrow{\tau_{\max }}(c)\left(s^{\prime}\right)$ for every $c \in C_{\max }$, which contradicts (7.7). Consequently $C_{\max } \cap D_{\tau} \neq \emptyset$. Since $S^{\prime} \cap D_{\tau} \neq \emptyset$ and $\tau\left[C_{\max } \cup S^{\prime}\right]$ is primitive, we obtain $C_{\max } \cup S^{\prime} \subseteq D_{\tau}$.

Let $v \in V(\sigma) \backslash D_{\tau}$. Since $C_{\max } \subseteq D_{\tau}$, there is $e_{v} \in E(\sigma)$ such that $\left(v, C_{\max }\right)_{\sigma}=e_{v}$. Since $D_{\tau}$ is a clan of $\tau$ such that $D_{\tau} \supseteq C_{\max } \cup S^{\prime}$, we get $\left(v, S^{\prime}\right)_{\tau}=e_{v}$. Therefore, for each $v \in V(\sigma) \backslash D_{\tau}$, there is $e_{v} \in E(\sigma)$ such that

$$
\vec{\tau}(v)_{\left\lceil S^{\prime}\right.}=\overline{e_{v}} \quad(\text { see }(7.6)) .
$$

Since $A_{0}$ and $A_{1}$ are distinct elements of $E(\sigma)^{S^{\prime}} \backslash\{\bar{e}: e \in E(\sigma)\}$, it follows from (7.9) that $V(\sigma) \backslash C(\sigma) \subseteq D_{\tau}$.

Lastly, consider $C \in \mathcal{C}$ omplete $(\sigma) \backslash\left\{C_{\max }\right\}$. First, suppose for a contradiction that $C \cap D_{\tau}=\emptyset$. There is $e \in E(\sigma)$ such that $\left(C, C_{\max }\right)_{\sigma}=e$. Since $D_{\tau}$ is a clan of $\tau$ such that $D_{\tau} \supseteq C_{\max } \cup S^{\prime}$, we get $\left(C, S^{\prime}\right)_{\tau}=e$, that is, $\vec{\tau}(c)_{\left\lceil S^{\prime}\right.}=\bar{e}$ for every $c \in C$. Therefore, for every $c \in C$, we have $B_{C}(c)=\bar{e}$, which contradicts (7.8). Thus $C \cap D_{\tau} \neq \emptyset$. Let $d \in C \cap D_{\tau}$. Second, suppose for a contradiction that $C \backslash D_{\tau} \neq \emptyset$. Given $c \in C \backslash D_{\tau}$, we obtain $c \longleftrightarrow_{\tau}\{d\} \cup S^{\prime}$. Since $\left(b, b^{\prime}\right)_{\sigma}=e_{C}$ for distinct $b, b^{\prime} \in C$, we obtain $\left(c,\{d\} \cup S^{\prime}\right)_{\tau}=e_{C}$. It follows from (7.9) that $\vec{\tau}(c)_{\left\lceil S^{\prime}\right.}=\overline{e_{C}}$, that is, $B_{C}(c)=\overline{e_{C}}$, which contradicts (7.8). Therefore $C \subseteq D_{\tau}$.

Consequently $C(\sigma) \subseteq D_{\tau}$, and hence $D_{\tau}=V(\sigma) \cup S^{\prime}$.

The next result follows from Proposition 7.3 and Theorem 7.4.

Corollary 7.5. For a reversible 2-structure $\sigma$ such that $2 \leq|E(\sigma)| \leq c(\sigma)<$ $\aleph_{0}$, we have $\left\lceil\log _{|E(\sigma)|}(c(\sigma))\right\rceil \leq p(\sigma) \leq\left\lceil\log _{|E(\sigma)|}(c(\sigma)+1)\right\rceil$. Consequently, if $c(\sigma) \notin\left\{|E(\sigma)|^{k}: k \geq 1\right\}$, then $p(\sigma)=\left\lceil\log _{|E(\sigma)|}(c(\sigma))\right\rceil$.

Let $\sigma$ be a reversible 2-structure such that $2 \leq|E(\sigma)|<\aleph_{0}$ and $c(\sigma)=$ $\left(|E(\sigma)|^{k}\right.$ where $k \geq 1$. By Corollary $7.5, p(\sigma)=k$ or $k+1$. We prove in Theorem 7.8 below that

$$
p(\sigma)=k+1 \text { if and only if } \sigma \text { admits exactly }|E(\sigma)|^{k} \text { isolated vertices. }
$$

The proofs of the next three results are adapted from that for finite graphs [2]. We begin by proving (7.10) from right to left.

Lemma 7.6. Let $\sigma$ be a reversible 2-structure such that $2 \leq|E(\sigma)|<\aleph_{0}$ and $c(\sigma)=|E(\sigma)|^{k}$ where $k \geq 1$. If $\sigma$ admits exactly $|E(\sigma)|^{k}$ isolated vertices, then $p(\sigma)=k+1$. 
Proof. Let $u$ and $v$ be distinct isolated vertices of $\sigma$. There exists a symmetric class $e$ of $\sigma$ such that $(u, v)_{\sigma}=e$. Recall that for each isolated vertex $w$ of $\sigma$, we have $(w, V(\sigma) \backslash\{w\})_{\sigma}=e$.

Let $S^{\prime}$ be a set such that $S^{\prime} \cap V(\sigma)=\emptyset$ and $\left|S^{\prime}\right|=k$. We consider the function

$$
\begin{aligned}
\bar{e}: & S^{\prime} \\
s^{\prime} & \longmapsto e(\sigma)
\end{aligned}
$$

Let $\tau$ be a faithful extension of $\sigma$ defined on $V(\sigma) \cup S^{\prime}$. Consider the function

$$
\begin{array}{cl}
A:\{v \in V(\sigma): v \text { isolated }\} & \longrightarrow E(\sigma)^{S^{\prime}} \\
v & \longmapsto \vec{\tau}(v)_{\left\lceil S^{\prime}\right.} .
\end{array}
$$

Since $\sigma$ admits exactly $|E(\sigma)|^{k}$ isolated vertices, that is, $\sigma$ admits exactly $\left|E(\sigma)^{S^{\prime}}\right|$ isolated vertices, we have either $A$ is not injective or there exists an isolated vertex $v$ of $\sigma$ such that $\vec{\tau}(v)_{\Gamma S^{\prime}}=\bar{e}$. In the second instance, $V(\tau) \backslash\{v\}$ is a clan of $\tau$. In the first, there exist distinct isolated vertices $w$ and $w^{\prime}$ of $\sigma$ such that $\vec{\tau}(w)_{\left\lceil S^{\prime}\right.}=\vec{\tau}\left(w^{\prime}\right)_{\left\lceil S^{\prime}\right.}$. It follows that $\left\{w, w^{\prime}\right\}$ is a clan of $\tau$. In both instances, $\tau$ is imprimitive. Therefore $p(\sigma) \neq k$. By Corollary 7.5, $p(\sigma)=k+1$.

Now, we show (7.10) from left to right when $|E(\sigma)|=2$ and $k=1$.

Proposition 7.7. Let $\sigma$ be a reversible 2-structure such that $c(\sigma)=|E(\sigma)|=$ 2. If $p(\sigma)=2$, then $\sigma$ possesses exactly two isolated vertices.

Proof. Since $|E(\sigma)|=2$ and $c(\sigma)=2, \sigma$ is symmetric. We obtain $\mathcal{L}$ inear $(\sigma)=$ $\emptyset$ and $|C|=2$ for each $C \in \mathcal{C}$ omplete $(\sigma)$. Denote the elements of $E(\sigma)$ by $e_{0}$ and $e_{1}$. By Proposition 4.2, $\sigma$ admits a traverse $T$, and $T$ admits a dense bicoloring $\beta$ by Proposition 4.12.

Consider $a \notin V(\sigma)$. Given $P \in \mathcal{P}$ rimitive $(\sigma)$, it follows from Lemma 5.5 that there is a primitive and faithful extension $\tau_{P}$ of $\sigma[P]$ defined on $P \cup\{a\}$. By Theorem 3.7, the elements of $\mathcal{C}$ omplete $(\sigma) \cup \mathcal{P}$ rimitive $(\sigma)$ are pairwise disjoint. Therefore, we can associate with $\beta$ a faithful extension $\tau_{\beta}$ of $\sigma$ to $V(\sigma) \cup\{a\}$ satisfying

- for each $C \in \mathcal{C}$ omplete $(\sigma), a \longleftrightarrow \tau_{\tau_{\beta}} C$;

- for each $P \in \mathcal{P}$ rimitive $(\sigma), \tau[P]=\tau_{P}$;

- for each $v \in V(\sigma) \backslash(C(\sigma) \cup P(\sigma)),(v, a)_{\tau_{\beta}}=e_{\beta(v)}$.

Since $p(\sigma)=2, \tau_{\beta}$ admits a nontrivial clan $D_{\beta}$. It follows from Corollary 5.8 applied with $\mathscr{F}=\mathcal{P}$ rimitive $(\sigma)$ that

- $a \in D_{\beta}$; 
- for each $C \in \mathcal{C}$ omplete $(\sigma), C \cap\left(D_{\beta} \backslash\{a\}\right) \neq \emptyset$;

- $P(\sigma) \subseteq D_{\beta} \backslash\{a\}$.

For each $C \in \mathcal{C}$ omplete $(\sigma)$, we have $\left(D_{\beta} \backslash\{a\}\right) \cup C$ is a clan of $\sigma$ because $C \cap\left(D_{\beta} \backslash\{a\}\right) \neq \emptyset$. Hence

$$
\bigcup_{C \in \mathcal{C} \text { omplete }(\sigma)}\left(\left(D_{\beta} \backslash\{a\}\right) \cup C\right) \text {, that is, }\left(D_{\beta} \backslash\{a\}\right) \cup C(\sigma)
$$

is a clan of $\sigma$. It follows that $\left(D_{\beta} \backslash\{a\}\right) \cup C(\sigma)$ is an inclusive clan of $\sigma$. Similarly, since $1-\beta$ is also a dense bicoloring of $T, \tau_{1-\beta}$ admits a nontrivial clan $D_{1-\beta}$, and $\left(D_{1-\beta} \backslash\{a\}\right) \cup C(\sigma)$ is an inclusive clan of $\sigma$. By Corollary 6.8, $\left(D_{\beta} \backslash\{a\}\right) \cup C(\sigma)$ and $\left(D_{1-\beta} \backslash\{a\}\right) \cup C(\sigma)$ are comparable under inclusion. For instance, assume that

$$
\left(D_{1-\beta} \backslash\{a\}\right) \cup C(\sigma) \subseteq\left(D_{\beta} \backslash\{a\}\right) \cup C(\sigma) .
$$

For a contradiction, suppose that there is $v \in V(\sigma) \backslash\left(\left(D_{\beta} \backslash\{a\}\right) \cup C(\sigma)\right)$. We get $v \notin C(\sigma) \cup P(\sigma)$. Hence $(v, a)_{\tau_{\beta}}=e_{\beta(v)}$ and $(v, a)_{\tau_{1-\beta}}=e_{1-\beta(v)}$. It follows that $\left(v, D_{\beta} \backslash\{a\}\right)_{\sigma}=e_{\beta(v)}$ and $\left(v, D_{\beta} \backslash\{a\}\right)_{\sigma}=e_{1-\beta(v)}$, which is impossible. Consequently

$$
\left(D_{\beta} \backslash\{a\}\right) \cup C(\sigma)=V(\sigma) .
$$

Since $D_{\beta}$ is a nontrivial clan of $\tau_{\beta}$, there is $C \in \mathcal{C}$ omplete $(\sigma)$ such that $C \backslash\left(D_{\beta} \backslash\{a\}\right) \neq \emptyset$. Since $C \cap\left(D_{\beta} \backslash\{a\}\right) \neq \emptyset$ and $|C|=2$, there exist $c, d \in C$ such that $C \backslash\left(D_{\beta} \backslash\{a\}\right)=\{c\}$ and $C \cap\left(D_{\beta} \backslash\{a\}\right)=\{d\}$. Since $\sigma[C]$ is complete, there exists a symmetric class $e$ of $\sigma$ such that $(c, d)_{\sigma}=e$. We get $\left(c, D_{\beta}\right)_{\tau_{\beta}}=e$ because $D_{\beta}$ is a clan of $\tau$.

Finally, consider $v \in V(\sigma) \backslash\left(D_{\beta} \backslash\{a\}\right)$ such that $v \neq c$. Since $C \backslash\left(D_{\beta} \backslash\right.$ $\{a\})=\{c\}$, there exists $C^{\prime} \in \mathcal{C}$ omplete $(\sigma) \backslash\{C\}$ such that $C^{\prime} \backslash\left(D_{\beta} \backslash\{a\}\right)=$ $\{v\}$. Since $\left(c, D_{\beta}\right)_{\tau_{\beta}}=e$, we obtain $\left(c, C^{\prime} \cap\left(D_{\beta} \backslash\{a\}\right)\right)_{\sigma}=e$. Thus $(c, v)_{\sigma}=e$ because $C^{\prime}$ is a clan of $\sigma$. It follows that $c$ is an isolated vertex of $\sigma$. Since $C$ is a clan of $\sigma$, we obtain that all the elements of $C$ are isolated vertices of $\sigma$. In particular $\sigma$ has at least two isolated vertices. Clearly $\{v \in V(\sigma): v$ isolated $\}$ is a clan of $\sigma$ such that $\sigma[\{v \in V(\sigma): v$ isolated $\}]$ is complete. By maximality of $C$, we obtain $C=\{v \in V(\sigma): v$ isolated $\}$.

We complete the proof of (7.10) as follows.

Theorem 7.8. For a reversible 2-structure $\sigma$ such that $2 \leq|E(\sigma)|<\aleph_{0}$ and $c(\sigma)=|E(\sigma)|^{k}$ where $k \geq 1, p(\sigma)=k+1$ if and only if $\sigma$ admits exactly $|E(\sigma)|^{k}$ isolated vertices. 
Proof. By Lemma 7.6 and Proposition 7.7, it suffices to establish (7.10) from left to right when $c(\sigma) \geq 3$. Hence consider a reversible 2-structure $\sigma$ such that $2 \leq|E(\sigma)|<\aleph_{0}, c(\sigma)=|E(\sigma)|^{k}$ (where $k \geq 1$ ), $c(\sigma) \geq 3$ and $p(\sigma)=k+1$. For convenience, set

$$
\mathcal{C}_{\max }(\sigma)=\{C \in \mathcal{C} \text { omplete }(\sigma):|C|=c(\sigma)\} .
$$

For each $C \in \mathcal{C}_{\max }(\sigma)$, choose $^{2} w_{C} \in C$. Set

$$
W=\left\{w_{C}: C \in \mathcal{C}_{\max }(\sigma)\right\} .
$$

We prove that

$$
c(\sigma-W)=|E(\sigma)|^{k}-1 .
$$

Let $C \in \mathcal{C}_{\max }(\sigma)$. By Theorem 3.7, the elements of $\mathcal{C}_{\max }(\sigma)$ are pairwise disjoint. Thus

$$
C \backslash W=C \backslash\left\{w_{C}\right\} .
$$

Clearly $(\sigma-W)\left[C \backslash\left\{w_{C}\right\}\right]$ is complete. Furthermore $C \backslash\left\{w_{C}\right\}$ is a clan of $\sigma-W$. Therefore $2^{k}-1=\left|C \backslash\left\{w_{C}\right\}\right| \leq c(\sigma-W)$.

Now, we demonstrate that $c(\sigma-W)<c(\sigma)$. Consider $C^{\prime} \in \mathcal{C}$ omplete $(\sigma-$ $W)$ such that $\left|C^{\prime}\right|=c(\sigma-W)$. We show that $C^{\prime}$ is a clan of $\sigma$. We have to verify that $w_{C} \longleftrightarrow{ }_{\sigma} C^{\prime}$ for each $C \in \mathcal{C}_{\max }(\sigma)$. Given $C \in \mathcal{C}_{\max }(\sigma)$, we distinguish the following two cases.

- First, suppose that there exists $v \in\left(C \backslash\left\{w_{C}\right\}\right) \backslash C^{\prime}$. We have $v \longleftrightarrow_{\sigma} C^{\prime}$. Since $\sigma[C]$ is complete, $\left\{v, w_{C}\right\}$ is a clan of $\sigma[C]$. Thus $\left\{v, w_{C}\right\}$ is a clan of $\sigma$, and hence $w_{C} \longleftrightarrow{ }_{\sigma} C^{\prime}$.

- Second, suppose that $C \backslash\left\{w_{C}\right\} \subseteq C^{\prime}$. Clearly $w_{C} \longleftrightarrow_{\sigma} C^{\prime}$ when $C \backslash\left\{w_{C}\right\}=C^{\prime}$. Suppose that $C^{\prime} \backslash\left(C \backslash\left\{w_{C}\right\}\right) \neq \emptyset$. Since $\sigma\left[C^{\prime}\right]$ is complete, there exists $e_{C^{\prime}} \in E(\sigma)$ such that $\left(c^{\prime}, d^{\prime}\right)=e_{C^{\prime}}$ for distinct elements $c^{\prime}$ and $d^{\prime}$ of $C^{\prime}$. We have $\left(C \backslash\left\{w_{C}\right\}, C^{\prime} \backslash C\right)_{\sigma-W}=e_{C^{\prime}}$. Since $C$ is a clan of $\sigma$, we get

$$
\left(w_{C}, C^{\prime} \backslash C\right)_{\sigma}=e_{C^{\prime}} .
$$

\footnotetext{
${ }^{2}$ It follows from the Axiom of Ultrafilter that there exists a linear order $L$ defined on $V(\sigma)$. For each $C \in \mathcal{C}_{\max }(\sigma), L[C]$ is a finite linear order. Hence $L[C]$ admits a smallest element. For instance, we can choose the smallest element of $L[C]$ for $w_{C}$.
} 
Furthermore, we have $\left|C \backslash\left\{w_{C}\right\}\right| \geq 2$ because $|C|=c(\sigma)$ and $c(\sigma) \geq 3$. Since $C \backslash\left\{w_{C}\right\} \subseteq C^{\prime}$ and $\sigma[C]$ is complete, we obtain also that $(c, d)=$ $e_{C^{\prime}}$ for distinct elements $c$ and $d$ of $C$. Therefore $\left(w_{C}, C \backslash\left\{w_{C}\right\}\right)_{\sigma}=e_{C^{\prime}}$. Since $C \backslash\left\{w_{C}\right\} \subseteq C^{\prime}$, it follows from (7.13) that $\left(w_{C}, C^{\prime}\right)_{\sigma}=e_{C^{\prime}}$.

Consequently, $C^{\prime}$ is a clan of $\sigma$. Since $\sigma\left[C^{\prime}\right]$ is complete, it follows from Lemma 3.2 that there is $D \in \mathcal{C}$ omplete $(\sigma)$ such that $D \supseteq C^{\prime}$. If $D \notin \mathcal{C}_{\max }(\sigma)$, then $\left|C^{\prime}\right| \leq|D|<c(\sigma)$. If $D \in \mathcal{C}_{\max }(\sigma)$, then $C^{\prime} \subseteq D \backslash\left\{w_{D}\right\}$, and hence $\left|C^{\prime}\right|<|D|=c(\sigma)$. In both instances, we obtain $\left|C^{\prime}\right|<c(\sigma)$. It follows that $c(\sigma-W)<c(\sigma)$ because $\left|C^{\prime}\right|=c(\sigma-W)$. Consequently (7.11) holds.

By Corollary 7.5, $p(\sigma-W)=k$. Thus there exists a primitive and faithful extension $\tau^{\prime}$ of $\sigma-W$ such that $\left|V\left(\tau^{\prime}\right) \backslash(V(\sigma) \backslash W)\right|=k$. We extend $\tau^{\prime}$ to $V\left(\tau^{\prime}\right) \cup W$ as follows. Let $C \in \mathcal{C}_{\max }(\sigma)$. Consider the function

$$
\begin{aligned}
A_{C}: C \backslash\left\{w_{C}\right\} & \longrightarrow E\left(\tau^{\prime}\right) V\left(\tau^{\prime}\right) \backslash(V(\sigma) \backslash W) \\
v & \longmapsto \overrightarrow{\tau^{\prime}}(v) \uparrow V\left(\tau^{\prime}\right) \backslash(V(\sigma) \backslash W)
\end{aligned}
$$

Since $\tau^{\prime}$ is primitive, $A_{C}$ is injective. Furthermore, since $\left|C \backslash\left\{w_{C}\right\}\right|=$ $|E(\sigma)|^{k}-1$ and $\left|E\left(\tau^{\prime}\right)^{V\left(\tau^{\prime}\right) \backslash(V(\sigma) \backslash W)}\right|=|E(\sigma)|^{k}$, there is a unique

$$
a_{C} \in E\left(\tau^{\prime}\right)^{V\left(\tau^{\prime}\right) \backslash(V(\sigma) \backslash W)}
$$

such that

$$
a_{C} \neq A_{C}(v) \text { for each } v \in C \backslash\left\{w_{C}\right\} \text {. }
$$

Consider the faithful extension $\tau$ of $\tau^{\prime}$ defined on $V\left(\tau^{\prime}\right) \cup W$ by

$$
\vec{\tau}\left(w_{C}\right) \uparrow V\left(\tau^{\prime}\right) \backslash(V(\sigma) \backslash W)=a_{C} \text { for each } C \in \mathcal{C}_{\max }(\sigma) .
$$

Since $p(\sigma)=k+1, \tau$ is not primitive. Consider a nontrivial clan $D_{\tau}$ of $\tau$. Now, we show the following assertion. Given distinct $C, C^{\prime} \in \mathcal{C}_{\max }(\sigma)$,

$$
\text { if } C \cap D_{\tau} \neq \emptyset \text { and } C^{\prime} \cap D_{\tau} \neq \emptyset \text {, then } V\left(\tau^{\prime}\right) \subseteq D_{\tau} \text {. }
$$

Indeed $D_{\tau} \cap V(\sigma)$ is a clan of $\sigma$. Furthermore $\left(D_{\tau} \cap V(\sigma)\right) \cap \widetilde{C} \neq \emptyset$ and $\left(D_{\tau} \cap V(\sigma)\right) \cap \widetilde{C^{\prime}} \neq \emptyset$. Since $\widetilde{C}$ and $\widetilde{C^{\prime}}$ are strong clans of $\sigma, D_{\tau} \cap V(\sigma)$ is comparable to $\widetilde{C}$ and $\widetilde{C^{\prime}}$ under inclusion.

Suppose, for a contradiction, that $D_{\tau} \cap V(\sigma) \subsetneq \widetilde{C}$ and $D_{\tau} \cap V(\sigma) \subsetneq \widetilde{C^{\prime}}$. It follows that $C \cap \widetilde{C^{\prime}} \neq \emptyset$. Since $\widetilde{C^{\prime}}$ is a strong clan of $\sigma, \widetilde{C^{\prime}} \subsetneq C$ or $C \subseteq \widetilde{C^{\prime}}$. In the first instance, $\widetilde{C^{\prime}}$ is a nontrivial strong clan of $\sigma[C]$, which contradicts 
the fact that $\sigma[C]$ is complete. Thus $C \subseteq \widetilde{C^{\prime}}$ and hence $\widetilde{C} \subseteq \widetilde{C^{\prime}}$. Similarly, we get $\widetilde{C^{\prime}} \subseteq \widetilde{C}$. Therefore $\widetilde{C^{\prime}}=\widetilde{C}$. It follows from Proposition 3.3 that $C=C^{\prime}$, but $C$ and $C^{\prime}$ are supposed to be distinct.

Consequently, $\widetilde{C} \subseteq\left(D_{\tau} \cap V(\sigma)\right)$ or $\widetilde{C^{\prime}} \subseteq\left(D_{\tau} \cap V(\sigma)\right)$. For instance, assume that $\widetilde{C} \subseteq\left(D_{\tau} \cap V(\sigma)\right)$. We get $\left(D_{\tau} \cap V\left(\tau^{\prime}\right)\right) \supseteq(\widetilde{C} \backslash W) \supseteq(C \backslash W)$ and $C \backslash W=C \backslash\left\{w_{C}\right\}$ by (7.12). Since $\tau^{\prime}$ is primitive and $\left|C \backslash\left\{w_{C}\right\}\right| \geq 2$, we obtain $V\left(\tau^{\prime}\right) \subseteq D_{\tau}$. It follows that (7.15) holds.

Clearly $D_{\tau} \cap V\left(\tau^{\prime}\right)$ is a clan of $\tau^{\prime}$. Since $\tau^{\prime}$ is primitive, we have $\mid D_{\tau} \cap$ $V\left(\tau^{\prime}\right) \mid \leq 1$ or $D_{\tau} \supseteq V\left(\tau^{\prime}\right)$. For a contradiction, suppose that $\left|D_{\tau} \cap V\left(\tau^{\prime}\right)\right| \leq 1$. Since $D_{\tau}$ is a nontrivial clan of $\tau$, there is $C \in \mathcal{C}_{\max }(\sigma)$ such that $w_{C} \in D_{\tau}$. It follows from (7.15) that

$$
C^{\prime} \cap D_{\tau}=\emptyset \text { for each } C^{\prime} \in \text { Complete }_{\max }(\sigma) \backslash\{C\} .
$$

Thus $D_{\tau} \cap W=\left\{w_{C}\right\}$. Since $\left|D_{\tau} \cap V\left(\tau^{\prime}\right)\right| \leq 1$, there exists $v \in V\left(\tau^{\prime}\right)$ such that $D_{\tau} \cap V\left(\tau^{\prime}\right)=\{v\}$. Clearly $D_{\tau}=\left\{v, w_{C}\right\}$, and we distinguish the following two cases obtaining a contradiction in each case.

- Suppose that $v \in V(\sigma-W)$. We obtain that $\left\{v, w_{C}\right\}$ is a clan of $\sigma$. Since $\sigma\left[\left\{v, w_{C}\right\}\right]$ is complete or linear, it follows from Lemma 3.2 that there exists $C^{\prime} \in \mathcal{C}$ omplete $(\sigma) \cup \mathcal{L}$ inear $(\sigma)$ such that $C^{\prime} \supseteq\left\{v, w_{C}\right\}$. Since $C, C^{\prime} \in\left\{D \in\left(V(\sigma) / \simeq_{\sigma}\right):|D| \geq 2\right\}$ by Theorem 3.7, we get $C=C^{\prime}$. Consequently we obtain

$$
\vec{\tau}\left(w_{C}\right)_{\mid V\left(\tau^{\prime}\right) \backslash(V(\sigma) \backslash W)}=\vec{\tau}(v)_{\mid V\left(\tau^{\prime}\right) \backslash(V(\sigma) \backslash W)},
$$

that is, $A_{C}(v)=a_{C}$, which contradicts (7.14).

- Suppose that $v \in V\left(\tau^{\prime}\right) \backslash V(\sigma-W)$. Consider the symmetric class $e_{C}$ of $\sigma$ such that $(c, d)_{\sigma}=e_{C}$ for distinct $c, d \in C$. We have $\left(w_{C}, C \backslash\right.$ $\left.\left\{w_{C}\right\}\right)_{\sigma}=e_{C}$. Since $\left\{v, w_{C}\right\}$ is a clan of $\tau$, we get $\left(v, C \backslash\left\{w_{C}\right\}\right)_{\tau^{\prime}}=e_{C}$. Since $A_{C}$ is injective, the function

$$
\begin{aligned}
C \backslash\left\{w_{C}\right\} & \longrightarrow E\left(\tau^{\prime}\right)^{\left(\left(V\left(\tau^{\prime}\right) \backslash(V(\sigma) \backslash W)\right) \backslash\{v\}\right)} \\
u & \longmapsto \overrightarrow{\tau^{\prime}}(u)_{\uparrow\left(V\left(\tau^{\prime}\right) \backslash(V(\sigma) \backslash W)\right) \backslash\{v\}}
\end{aligned}
$$

is injective also. Therefore, we obtain $|E(\sigma)|^{k}-1 \leq|E(\sigma)|^{k-1}$, which does not hold when $|E(\sigma)|^{k} \geq 3$.

Consequently $V\left(\tau^{\prime}\right) \subseteq D_{\tau}$. Since $D_{\tau}$ is a nontrivial module of $\tau$, there exists $C \in \mathcal{C}_{\max }(\sigma)$ such that $w_{C} \notin D_{\tau}$. Consider the symmetric class $e_{C}$ of $\sigma$ such that $(c, d)_{\sigma}=e_{C}$ for distinct $c, d \in C$. We have $\left(w_{C}, C \backslash\left\{w_{C}\right\}\right)_{\sigma}=e_{C}$ 
and hence $\left(w_{C}, V\left(\tau^{\prime}\right)\right)_{\tau}=e_{C}$. In particular $\left(w_{C}, V(\sigma-W)\right)_{\sigma}=e_{C}$. Given $C^{\prime} \in \mathcal{C}_{\max }(\sigma) \backslash\{C\}$, we obtain $\left(w_{C}, C^{\prime} \backslash\left\{w_{C^{\prime}}\right\}\right)_{\sigma}=e_{C}$. Since $C^{\prime}$ is a clan of $\sigma$, we get $\left(w_{C}, w_{C^{\prime}}\right)_{\sigma}=e_{C}$. It follows that $w_{C}$ is an isolated vertex of $\sigma$. As at the end of the proof of Proposition 7.7, we conclude by $C=\{v \in V(\sigma)$ : $v$ isolated $\}$.

Given Theorems 7.1, 7.2 and 7.4, we complete this section by considering reversible 2-structures $\sigma$ such that $c(\sigma)>|E(\sigma)| \geq 2$ and $c(\sigma) \geq \aleph_{0}$, that is, reversible 2-structures $\sigma$ such that $|E(\sigma)| \geq 2$ and $\mathfrak{l o g}_{|E(\sigma)|}(c(\sigma)) \geq \aleph_{0}$.

Theorem 7.9. Consider a reversible 2-structure $\sigma$ such that $|E(\sigma)| \geq 2$. If $\mathfrak{l o g}_{|E(\sigma)|}(c(\sigma)) \geq \aleph_{0}$, then $p(\sigma)=\mathfrak{l o g}_{|E(\sigma)|}(c(\sigma))$.

Proof. By Proposition 7.3, it suffices to construct a primitive and faithful extension $\tau$ of $\sigma$ such that $|V(\tau) \backslash V(\sigma)|=\log _{|E(\sigma)|}(c(\sigma))$. Let $S^{\prime}$ be a set such that $S^{\prime} \cap V(\sigma)=\emptyset$ and $\left|S^{\prime}\right|=\mathfrak{l o g}_{|E(\sigma)|}(c(\sigma))$. Thus $|E(\sigma)|^{\left|S^{\prime}\right|} \geq c(\sigma)$. We use Lemma 5.3 as follows. It is easy to construct a primitive and reversible 2-structure $\sigma^{\prime}$ defined on $S^{\prime}$ such that $\left|E\left(\sigma^{\prime}\right)\right|=2$ and $\sigma^{\prime}$ is symmetric or asymmetric. Hence we can assume that $\mid\left\{e^{\prime} \in E\left(\sigma^{\prime}\right): e^{\prime}\right.$ asymmetric $\} \mid \leq$ $\mid\{e \in E(\sigma): e$ asymmetric $\} \mid$ and $\mid\left\{e^{\prime} \in E\left(\sigma^{\prime}\right): e^{\prime}\right.$ symmetric $\}|\leq|\{e \in E(\sigma)$ : $e$ symmetric $\} \mid$. Moreover, since $\left|S^{\prime}\right|=\mathfrak{l o g}_{|E(\sigma)|}(c(\sigma)) \geq \aleph_{0}$, we have $|E(\sigma)|<$ $c(\sigma)$ and hence $|E(\sigma)|^{\left|S^{\prime}\right|}>|E(\sigma)|$. Finally, consider $C \in \mathcal{C}$ omplete $(\sigma)$. We have $|C| \leq c(\sigma) \leq|E(\sigma)|^{\left|S^{\prime}\right|}$. By Lemma 5.3 applied with $S=C$, there exists an extension $\tau_{C}$ of $\sigma$ and $\sigma^{\prime}$ to $V(\sigma) \cup V\left(\sigma^{\prime}\right)$ such that $\tau_{C}$ is a faithful extension of $\sigma$ and $\tau_{C}\left[C \cup V\left(\sigma^{\prime}\right)\right]$ is primitive.

Let $a \notin V\left(\sigma^{\prime}\right)$. By Lemma 5.5, there exist distinct, primitive and faithful extensions $\sigma_{0}^{\prime \prime}$ and $\sigma_{1}^{\prime \prime}$ of $\sigma^{\prime}$ to $V\left(\sigma^{\prime}\right) \cup\{a\}$. Set $A_{0}=\overrightarrow{\sigma_{0}^{\prime \prime}}(a)$ and $A_{1}=\overrightarrow{\sigma_{1}^{\prime \prime}}(a)$. We get $A_{0}, A_{1}: S^{\prime} \longrightarrow E\left(\sigma^{\prime}\right)$ and $A_{0} \neq A_{1}$. As in the proof of Lemma 5.3, we identify the elements of $E\left(\sigma^{\prime}\right)$ to elements of $E(\sigma)$. In this way, $A_{0}, A_{1} \in$ $E(\sigma)^{S^{\prime}}$. By Proposition 4.2, $\sigma$ admits a traverse $T$, and $T$ admits a dense bicoloring $\beta$ by Proposition 4.12. Furthermore, the elements of $\mathcal{C}$ omplete $(\sigma)$ are pairwise disjoint by Theorem 3.7. Therefore, we can consider the faithful extension $\tau$ of $\sigma$ defined on $V(\sigma) \cup S^{\prime}$ satisfying

- for each $C \in \mathcal{C}$ omplete $(\sigma), \tau\left[C \cup S^{\prime}\right]=\tau_{C}\left[C \cup V\left(\sigma^{\prime}\right)\right]$;

- for each $v \in V(\sigma) \backslash C(\sigma), \vec{\tau}(v)_{\uparrow S^{\prime}}=A_{\beta(v)}$.

For each $C \in \mathcal{C}$ omplete $(\sigma), \tau\left[C \cup S^{\prime}\right]=\tau_{C}\left[C \cup V\left(\sigma^{\prime}\right)\right]$ is primitive. Moreover, consider $v \in V(\sigma) \backslash C(\sigma)$. Since $\vec{\tau}(v)_{\uparrow S^{\prime}}=A_{\beta(v)}=\overrightarrow{\sigma_{\beta(v)}^{\prime \prime}}(a), \tau\left[S^{\prime} \cup\{v\}\right]$ is primitive. Consequently, $\tau\left[X \cup S^{\prime}\right]$ is primitive for each $X \in \mathcal{C}$ omplete $(\sigma) \cup$ $\{\{v\}: v \in V(\sigma) \backslash C(\sigma)\}$. 
We prove that $\tau$ is primitive. Let $D_{\tau}$ be a clan of $\tau$ such that $\left|D_{\tau}\right| \geq 2$. We have to show that $D_{\tau}=V(\sigma) \cup S^{\prime}$. Since $\tau\left[C \cup S^{\prime}\right]$ is primitive for each $C \in \mathcal{C}$ omplete $(\sigma)$, (5.1a) holds (see Lemma 5.7). By applying Lemma 5.7 with $\mathscr{F}=\emptyset$, we obtain $D_{\tau} \cap S^{\prime} \neq \emptyset$. Since $\tau\left[S^{\prime}\right]=\sigma^{\prime}$ is primitive, we have $\left|D_{\tau} \cap S^{\prime}\right|=1$ or $D_{\tau} \cap S^{\prime}=S^{\prime}$. Suppose for a contradiction that the first instance holds. Since $\left|D_{\tau}\right| \geq 2$, there is $X \in \mathcal{C}$ omplete $(\sigma) \cup\{\{v\}$ : $v \in V(\sigma) \backslash C(\sigma)\}$ such that $D_{\tau} \cap X \neq \emptyset$. Since $\tau\left[X \cup S^{\prime}\right]$ is imprimitive, we obtain $D_{\tau} \supseteq X \cup S^{\prime}$, which contradicts $\left|D_{\tau} \cap S^{\prime}\right|=1$. Consequently, we have $D_{\tau} \cap S^{\prime}=S^{\prime}$. Let $X \in \mathcal{C}$ omplete $(\sigma) \cup\{\{v\}: v \in V(\sigma) \backslash C(\sigma)\}$. Since $\tau\left[X \cup S^{\prime}\right]$ is primitive, we obtain $D_{\tau} \cap\left(X \cup S^{\prime}\right)=\left(X \cup S^{\prime}\right)$. Therefore $X \subseteq D_{\tau}$ for every $X \in \mathcal{C}$ omplete $(\sigma) \cup\{\{v\}: v \in V(\sigma) \backslash C(\sigma)\}$. It follows that $D_{\tau}=V(\sigma) \cup S^{\prime}$.

\section{Primitive bounds of non-reversible 2-structures}

Before extending our results from reversible 2-structures to non-reversible ones, we discuss the necessity of Conditions (1.1) and (1.2) (see Definition 1.1). Given $n>0, L_{n}$ denotes the usual linear order on $\{0, \ldots, n\}$. When $n$ is even, there does not exist a primitive tournament which is a 1extension of $L_{n}$. On the other hand, there is a primitive tournament which is a 2-extension of $L_{n}$ (see [24]). The 2-structure $\sigma\left(L_{n}\right)$ is defined on $\{0, \ldots, n\}$ by $E\left(\sigma\left(L_{n}\right)\right)=\left\{e, e^{\star}\right\}$, where $e=\{(p, q): 0 \leq p<q \leq n\}$. Let $\tau$ be an extension of $\sigma\left(L_{n}\right)$ to $\{0, \ldots, n+1\}$ satisfying

- for $0 \leq i \leq n-1$ such that $i$ is even, $(n+1, i) \equiv_{\tau}(0,1)$ and $(i, n+1) \equiv_{\tau}$ $(1,0)$;

- for $0 \leq i \leq n-1$ such that $i$ is odd, $(i, n+1) \equiv_{\tau}(0,1)$ and $(n+1, i) \equiv_{\tau}$ $(1,0)$;

- $(n, n+1) \equiv_{\tau}(n+1, n)$.

It is simple to verify that $\tau$ is primitive. Moreover, $\tau$ is not identifiable with a tournament because $(n, n+1) \equiv_{\tau}(n+1, n)$. If $(n, n+1) \equiv_{\tau}(0,1)$ and $(n, n+1) \equiv_{\tau}(1,0)$, then $|E(\tau)|=3$. Since $\left|E\left(L_{n}\right)\right|=2, \sigma\left(L_{n}\right) \hookrightarrow \tau$ is not bijective, that is, Condition (1.1) does not hold. Now, assume for instance that $(n, n+1) \equiv_{\tau}(0,1)$. We obtain $(n, n+1) \in\left(\sigma\left(L_{n}\right) \hookrightarrow \tau\right)(e)$. We have also $(n+1, n) \equiv_{\tau}(0,1)$ and hence $(n+1, n) \in\left(\sigma\left(L_{n}\right) \hookrightarrow \tau\right)(e)$. Thus

$$
(n, n+1) \in\left(\sigma\left(L_{n}\right) \hookrightarrow \tau\right)(e) \cap\left(\left(\sigma\left(L_{n}\right) \hookrightarrow \tau\right)(e)\right)^{\star},
$$

but $e \cap e^{\star}=\emptyset$. Therefore, Condition (1.2) does not hold.

Conditions (1.1) and (1.2) ensure that the faithful extensions of a 2structure $\sigma$ are 2-structures of the "same type" as $\sigma$. Also, they ensure that 
the faithful extensions of a reversible 2-structure are reversible as well (see the next lemma).

Lemma 8.1. Let $\sigma$ be a 2-structure with $|E(\sigma)| \geq 2$. Given a faithful extension $\tau$ of $\sigma, \sigma$ is reversible if and only if $\tau$ is.

Proof. To begin, assume that $\sigma$ is reversible. We prove that for every $e \in$ $E(\sigma)$,

$$
(\sigma \hookrightarrow \tau)\left(e^{\star}\right)=((\sigma \hookrightarrow \tau)(e))^{\star}
$$

Consider $e \in E(\sigma)$. Let $(u, v) \in((\sigma \hookrightarrow \tau)(e))^{\star}$. By Condition (1.1), there is $f_{(u, v)} \in E(\sigma)$ such that $(u, v)_{\tau}=(\sigma \hookrightarrow \tau)\left(f_{(u, v)}\right)$. We obtain $(u, v) \in(\sigma \hookrightarrow$ $\tau)\left(f_{(u, v)}\right) \cap((\sigma \hookrightarrow \tau)(e))^{\star}$. By Condition $(1.2), f_{(u, v)} \cap e^{\star} \neq \emptyset$. Since $\sigma$ is reversible, $e^{\star} \in E(\sigma)$ and hence $f_{(u, v)}=e^{\star}$. It follows that $(u, v)_{\tau}=(\sigma \hookrightarrow$ $\tau)\left(e^{\star}\right)$ for every $(u, v) \in((\sigma \hookrightarrow \tau)(e))^{\star}$. Thus

$$
((\sigma \hookrightarrow \tau)(e))^{\star} \subseteq(\sigma \hookrightarrow \tau)\left(e^{\star}\right)
$$

By applying (8.2) to $e^{\star} \in E(\sigma)$, we obtain $\left((\sigma \hookrightarrow \tau)\left(e^{\star}\right)\right)^{\star} \subseteq(\sigma \hookrightarrow \tau)(e)$, and hence $(\sigma \hookrightarrow \tau)\left(e^{\star}\right) \subseteq((\sigma \hookrightarrow \tau)(e))^{\star}$. Therefore (8.1) holds.

Now, consider $e_{\tau} \in E(\tau)$. By Condition (1.1), there is $e \in E(\sigma)$ such that $(\sigma \hookrightarrow \tau)(e)=e_{\tau}$. By $(8.1),\left(e_{\tau}\right)^{\star}=(\sigma \hookrightarrow \tau)\left(e^{\star}\right)$. Consequently, $\left(e_{\tau}\right)^{\star} \in E(\tau)$ for each $e_{\tau} \in E(\tau)$. It follows that $\tau$ is reversible.

Conversely, assume that $\tau$ is reversible. We observe the following fact. It follows from Condition (1.1) that

$$
(\sigma \hookrightarrow \tau)(e) \cap((V(\sigma) \times V(\sigma)) \backslash\{(v, v): v \in V(\sigma)\})=e
$$

for each $e \in E(\sigma)$. Consider $e \in E(\sigma)$. It suffices to show that $e^{\star} \in E(\sigma)$. Since $\tau$ is reversible and $(\sigma \hookrightarrow \tau)(e) \in E(\tau)$, we have $((\sigma \hookrightarrow \tau)(e))^{\star} \in E(\tau)$. By Condition (1.1), there is $f \in E(\sigma)$ such that $((\sigma \hookrightarrow \tau)(e))^{\star}=(\sigma \hookrightarrow$ $\tau)(f)$. Hence

$$
\begin{aligned}
((\sigma \hookrightarrow \tau)(e))^{\star} \cap & ((V(\sigma) \times V(\sigma)) \backslash\{(v, v): v \in V(\sigma)\}) \\
= & (\sigma \hookrightarrow \tau)(f) \cap((V(\sigma) \times V(\sigma)) \backslash\{(v, v): v \in V(\sigma)\}) .
\end{aligned}
$$

By $(8.3),(\sigma \hookrightarrow \tau)(f) \cap((V(\sigma) \times V(\sigma)) \backslash\{(v, v): v \in V(\sigma)\})=f$. Furthermore

$$
\begin{aligned}
& ((\sigma \hookrightarrow \tau)(e))^{\star} \cap((V(\sigma) \times V(\sigma)) \backslash\{(v, v): v \in V(\sigma)\}) \\
= & ((\sigma \hookrightarrow \tau)(e) \cap((V(\sigma) \times V(\sigma)) \backslash\{(v, v): v \in V(\sigma)\}))^{\star} \\
= & e^{\star} \quad \text { by }(8.3) .
\end{aligned}
$$

Therefore $e^{\star}=f$ and hence $e^{\star} \in E(\sigma)$. 
Lemma 8.2. Consider 2-structures $\sigma$ and $\tau$ such that $\tau$ is an extension of $\sigma$. If $\sigma$ and $\tau$ are reversible, then Condition (1.2) holds.

Proof. Consider $e, f \in E(\sigma)$ such that

$$
(\sigma \hookrightarrow \tau)(e) \cap((\sigma \hookrightarrow \tau)(f))^{\star} \neq \emptyset
$$

Since $(\sigma \hookrightarrow \tau)(f) \supseteq f$, we have $((\sigma \hookrightarrow \tau)(f))^{\star} \supseteq f^{\star}$. Since $\sigma$ is reversible, $f^{\star} \in E(\sigma)$, and we have $(\sigma \hookrightarrow \tau)\left(f^{\star}\right) \supseteq f^{\star}$. Since $\tau$ is reversible, $((\sigma \hookrightarrow$ $\tau)(f))^{\star} \in E(\tau)$. It follows that $((\sigma \hookrightarrow \tau)(f))^{\star}=(\sigma \hookrightarrow \tau)\left(f^{\star}\right)$. Since $\sigma \hookrightarrow \tau$ is injective, $(\sigma \hookrightarrow \tau)(e) \cap((\sigma \hookrightarrow \tau)(f))^{\star} \neq \emptyset$ implies $e=f^{\star}$.

Given a 2-structure $\sigma$, recall that $\sigma \wedge \sigma^{\star}$ is defined on $V(\sigma)$ by $E\left(\sigma \wedge \sigma^{\star}\right)=$ $\left\{e \cap f^{\star}: e, f \in E(\sigma), e \cap f^{\star} \neq \emptyset\right\}$. Clearly $\sigma \wedge \sigma^{\star}$ is reversible. Furthermore, $\sigma$ and $\sigma \wedge \sigma^{\star}$ share the same clans.

Proposition 8.3. Let $\sigma$ be a 2-structure.

1. If $\tau$ is a faithful extension of $\sigma$, then $\tau \wedge \tau^{\star}$ is a faithful extension of $\sigma \wedge \sigma^{\star}$.

2. If $\rho$ is a faithful extension of $\sigma \wedge \sigma^{\star}$, then there is a faithful extension $\tau$ of $\sigma$ such that $\rho=\tau \wedge \tau^{\star}$.

Proof. First, suppose that $\tau$ is a faithful extension of $\sigma$. Clearly $\tau \wedge \tau^{\star}$ is an extension of $\sigma \wedge \sigma^{\star}$. Consider an element $e \cap f^{\star}$ of $E\left(\sigma \wedge \sigma^{\star}\right)$, where $e, f \in E(\sigma)$ such that $e \cap f^{\star} \neq \emptyset$. Since $(\sigma \hookrightarrow \tau)(e) \supseteq e$ and $((\sigma \hookrightarrow \tau)(f))^{\star} \supseteq f^{\star}$, we have $(\sigma \hookrightarrow \tau)(e) \cap((\sigma \hookrightarrow \tau)(f))^{\star} \supseteq e \cap f^{\star}$. Furthermore $(\sigma \hookrightarrow \tau)(e) \cap((\sigma \hookrightarrow$ $\tau)(f))^{\star} \in E\left(\tau \wedge \tau^{\star}\right)$. Thus

$$
\left(\left(\sigma \wedge \sigma^{\star}\right) \hookrightarrow\left(\tau \wedge \tau^{\star}\right)\right)\left(e \cap f^{\star}\right)=(\sigma \hookrightarrow \tau)(e) \cap((\sigma \hookrightarrow \tau)(f))^{\star}
$$

for any $e, f \in E(\sigma)$ such that $e \cap f^{\star} \neq \emptyset$.

Now, we prove that Condition (1.1) holds for the extension $\tau \wedge \tau^{\star}$ of $\sigma \wedge \sigma^{\star}$. It suffices to show that $\left(\sigma \wedge \sigma^{\star}\right) \hookrightarrow\left(\tau \wedge \tau^{\star}\right)$ is surjective. Consider $e_{\tau \wedge \tau^{\star}} \in$ $E\left(\tau \wedge \tau^{\star}\right)$. There exist $e_{\tau}, f_{\tau} \in E(\tau)$ such that $e_{\tau \wedge \tau^{\star}}=e_{\tau} \cap\left(f_{\tau}\right)^{\star}$. Since $\sigma \hookrightarrow \tau$ is bijective, there exist $e_{\sigma}, f_{\sigma} \in E(\sigma)$ such that $e_{\tau}=(\sigma \hookrightarrow \tau)\left(e_{\sigma}\right)$ and $f_{\tau}=(\sigma \hookrightarrow \tau)\left(f_{\sigma}\right)$. Thus $e_{\tau \wedge \tau^{\star}}=(\sigma \hookrightarrow \tau)\left(e_{\sigma}\right) \cap\left((\sigma \hookrightarrow \tau)\left(f_{\sigma}\right)\right)^{\star}$. Since Condition (1.2) is satisfied by the extension $\tau$ of $\sigma$, we obtain $e_{\sigma} \cap\left(f_{\sigma}\right)^{\star} \neq \emptyset$. It follows from (8.4) that $e_{\tau \wedge \tau^{\star}}=\left(\left(\sigma \wedge \sigma^{\star}\right) \hookrightarrow\left(\tau \wedge \tau^{\star}\right)\right)\left(e_{\sigma} \cap\left(f_{\sigma}\right)^{\star}\right)$.

Lastly, since $\sigma \wedge \sigma^{\star}$ and $\tau \wedge \tau^{\star}$ are reversible, Condition (1.2) holds by Lemma 8.2. 
Second, suppose that $\rho$ is a faithful extension of $\sigma \wedge \sigma^{\star}$. For each $e \in E(\sigma)$, set

$$
\underline{e}=\bigcup_{\left\{f \in E(\sigma): e \cap f^{\star} \neq \emptyset\right\}}\left(\left(\sigma \wedge \sigma^{\star}\right) \hookrightarrow \rho\right)\left(e \cap f^{\star}\right) .
$$

Since $E(\rho)=\left\{\left(\left(\sigma \wedge \sigma^{\star}\right) \hookrightarrow \rho\right)\left(e \cap f^{\star}\right): e, f \in E(\sigma), e \cap f^{\star} \neq \emptyset\right\}$ is a partition of $(V(\rho) \times V(\rho)) \backslash\{(v, v): v \in V(\rho)\},\{\underline{e}: e \in E(\sigma)\}$ is also. Denote by $\tau$ the unique 2-structure defined on $V(\tau)=V(\rho)$ by $E(\tau)=\{\underline{e}: e \in E(\sigma)\}$.

Let $e \in E(\sigma)$. By $(8.5)$,

$$
\underline{e} \cap(V(\sigma) \times V(\sigma))=\bigcup_{\left\{f \in E(\sigma): e \cap f^{\star} \neq \emptyset\right\}}\left(\left(\sigma \wedge \sigma^{\star}\right) \hookrightarrow \rho\right)\left(e \cap f^{\star}\right) \cap(V(\sigma) \times V(\sigma)) .
$$

Since $\rho$ is an extension of $\sigma \wedge \sigma^{\star}$, we get $\left(\left(\sigma \wedge \sigma^{\star}\right) \hookrightarrow \rho\right)\left(e \cap f^{\star}\right) \cap(V(\sigma) \times$ $V(\sigma))=e \cap f^{\star}$ for each $f \in E(\sigma)$ such that $e \cap f^{\star} \neq \emptyset$. Thus

$$
\underline{e} \cap(V(\sigma) \times V(\sigma))=\bigcup_{\left\{f \in E(\sigma): e \cap f^{\star} \neq \emptyset\right\}} e \cap f^{\star}=e .
$$

Therefore, $\tau$ is an extension of $\sigma$ such that $(\sigma \hookrightarrow \tau)(e)=\underline{e}$. It follows that Condition (1.1) holds for the extension $\tau$ of $\sigma$. For Condition (1.2), consider $e, g \in E(\sigma)$ such that $(\sigma \hookrightarrow \tau)(e) \cap((\sigma \hookrightarrow \tau)(g))^{\star} \neq \emptyset$, that is, $\underline{e} \cap(\underline{g})^{\star} \neq \emptyset$. By (8.5), there exist $f \in E(\sigma)$ such that $e \cap f^{\star} \neq \emptyset$, and $h \in E(\sigma)$ such that $g \cap h^{\star} \neq \emptyset$ satisfying

$$
\left(\left(\sigma \wedge \sigma^{\star}\right) \hookrightarrow \rho\right)\left(e \cap f^{\star}\right) \cap\left(\left(\left(\sigma \wedge \sigma^{\star}\right) \hookrightarrow \rho\right)\left(g \cap h^{\star}\right)\right)^{\star} \neq \emptyset .
$$

Since Condition (1.2) holds for the extension $\rho$ of $\sigma \wedge \sigma^{\star}$, we obtain $(e \cap$ $\left.f^{\star}\right) \cap\left(g \cap h^{\star}\right)^{\star} \neq \emptyset$ and hence $e \cap g^{\star} \neq \emptyset$.

The next corollary is an immediate consequence of Proposition 8.3 and of the fact that for a 2-structure $\sigma, \sigma$ and $\sigma \wedge \sigma^{\star}$ share the same clans.

Corollary 8.4. Let $\sigma$ be a 2-structure.

1. If $\tau$ is a primitive faithful extension of $\sigma$, then $\tau \wedge \tau^{\star}$ is a primitive faithful extension of $\sigma \wedge \sigma^{\star}$.

2. If $\rho$ is a primitive faithful extension of $\sigma \wedge \sigma^{\star}$, then there is a primitive faithful extension $\tau$ of $\sigma$ such that $\rho=\tau \wedge \tau^{\star}$.

We conclude with the following.

Theorem 8.5. For every 2-structure $\sigma, p(\sigma)=p\left(\sigma \wedge \sigma^{\star}\right)$. 


\section{Acknowledgements}

The authors express their sincere gratitude to the referee for his careful reading of the first two versions of this paper, and all his comments which improve a lot this paper.

\section{References}

[1] Birnbaum, Z. W. and Esary, J. D. (1965). Modules of coherent binary systems. J. Soc. Indust. Appl. Math. 13 444-462.

[2] Boussaïri, A. and Ille, P. (2014). Determination of the prime bound of a graph. Contrib. Discrete Math. 9 46-62. MR3265751

[3] Brignall, R. (2007). Simplicity in relational structures and its application to permutation classes. Ph.D. Thesis, University of St Andrews.

[4] Brignall, R., Ruškuc, N. and Vatter, V. (2011). Simple extensions of combinatorial structures. Mathematika 57 193-214. MR2825233

[5] Capelle, C. (1997). Décompositions de Graphes et Permutations Factorisantes. Ph.D. Thesis, Université Montpellier II.

[6] Capelle, C., Habib, M. and de Montgolfier, F. (2002). Graph decompositions and factorizing permutations. Discrete Math. Theor. Comput. Sci. 5 55-70. MR1902414

[7] McConnell, R. and de Montgolfier, F. (2005). Linear-time modular decomposition of directed graphs. Discrete Appl. Math. 145 198-209. MR2113141

[8] Cournier, A. and Habib, M. (1993). An efficient algorithm to recognize prime undirected graphs. Lecture Notes in Computer Science 657212 224. MR1244139

[9] Courcelle, B. and Delhommé, C. (2008). The modular decomposition of countable graphs. Definition and construction in monadic second-order logic. Theoret. Comput. Sci. 394 1-38. MR2399790

[10] Ehrenfeucht, A., Harju, T. and Rozenberg, G. (1999). The Theory of 2-Structures, A Framework for Decomposition and Transformation of Graphs. World Scientific, Singapore. MR1712180

[11] Erdős, P., Fried, E., Hajnal, A. and Milner, E. C. (1972). Some remarks on simple tournaments. Algebra Universalis 2 238-245. MR0306034 
[12] Erdős, P., Hajnal, A. and Milner, E. C. (1972). Simple one point extensions of tournaments. Mathematika 19 57-62. MR0382059

[13] Fraïssé, R. (1953). On a decomposition of relations which generalizes the sum of ordering relations. Bull. Amer. Math. Soc. 59389.

[14] Fraïssé, R. (1984). L'intervalle en théorie des relations, ses généralisations, filtre intervallaire et clôture d'une relation. NorthHolland Math. Stud. 99 313-342. MR0779858

[15] Fried, E. and Laskar, H. (1971). Simple tournaments. Notices Amer. Math. Soc. 18395.

[16] Gallai, T. (1967). Transitiv orientierbare Graphen. Acta Math. Acad. Sci. Hungar. 18 25-66. MR0221974

[17] Giakoumakis, V. (1997). On the closure of graphs under substitution. Discrete Math. 177 83-97. MR1483440

[18] Habib, M., Paul, C. and Viennot, L. (1999). Partition Refinement techniques: an interesting tool kit. Internat. J. Found. Comput. Sci. 10 14-170. MR1759929

[19] Harju, T. and Rozenberg, G. (1994). Decomposition of infinite labeled 2-structures. Lecture Notes in Comput. Sci. 812 145-158. MR1286963

[20] Ille, P. (1997). Indecomposable graphs. Discrete Math. 173 71-78. MR1468841

[21] Ille, P. and Woodrow, R. E. (2009). Weakly partitive families on infinite sets. Contrib. Discrete Math. 4 54-80. MR2541988

[22] Kelly, D. (1985). Comparability graphs. NATO Adv. Sci. Inst. Ser. C Math. Phys. Sci. 147 3-40. MR0818492

[23] Maffray, F. and Preissmann, M. (2001). A translation of Tibor Gallai's paper: Transitiv orientierbare Graphen. In: Perfect Graphs, Wiley, New York, 25-66. MR1861357

[24] Moon, J.W. (1972). Embedding tournaments in simple tournaments. Discrete Math. 2 389-395. MR0302492

[25] Schmerl, J. H. and Trotter, W. T. (1993). Critically indecomposable partially ordered sets, graphs, tournaments and other binary relational structures. Discrete Math. 113 191-205. MR1212878

[26] Spinrad, J. (1992). P4-trees and substitution decomposition. Discrete Appl. Math. 39 263-291. MR1189472 
[27] Sumner, D. P. (1971). Indecomposable graphs. Ph.D. Thesis, University of Massachusetts. MR2620781

[28] Sumner, D. P. (1973). Graphs indecomposable with respect to the Xjoin. Discrete Math. 6 281-298. MR0325468

\section{Symbol glossary}

\begin{tabular}{|c|c|c|}
\hline Symbol & Notation & Page \\
\hline$(u, v)_{\sigma}$ & 2.1 & 548 \\
\hline$\left(W, W^{\prime}\right)_{\sigma}$ & 2.1 & 548 \\
\hline$(v, W)_{\sigma}$ & 2.1 & 548 \\
\hline$W \longleftrightarrow W^{\prime}$ & 2.1 & 548 \\
\hline$v \longleftrightarrow{ }_{\sigma} W$ & 2.1 & 548 \\
\hline$v \nLeftarrow \longleftrightarrow_{\sigma} W$ & 2.1 & 548 \\
\hline$\widehat{W}$ & 2.8 & 549 \\
\hline$\widetilde{W}$ & 2.8 & 549 \\
\hline$\simeq_{\sigma}$ & 3.5 & 552 \\
\hline$V(\sigma) / \simeq_{\sigma}$ & 3.6 & 552 \\
\hline$V_{1}(\sigma)$ & 3.8 & 552 \\
\hline$V_{\downarrow}(\sigma)$ & 3.8 & 552 \\
\hline$T$ & 4.4 & 553 \\
\hline$e_{X}$ & 4.5 & 554 \\
\hline$T_{X}$ & 4.6 & 554 \\
\hline$\vec{\sigma}$ & 5.2 & 559 \\
\hline$c(\sigma)$ & 1.4 & 546 \\
\hline $\mathcal{C l a n s}(\sigma)$ & 2.2 & 548 \\
\hline Complete $(\sigma)$ & 3.1 & 550 \\
\hline$C(\sigma)$ & 5.6 & 563 \\
\hline $\mathcal{G}$ allai $(\sigma)$ & 2.4 & 549 \\
\hline $\mathcal{L}$ inear $(\sigma)$ & 3.1 & 550 \\
\hline$L(\sigma)$ & 5.6 & 563 \\
\hline$p(\sigma)$ & 1.2 & 546 \\
\hline $\mathcal{P}$ rimitive $(\sigma)$ & 3.1 & 550 \\
\hline$P(\sigma)$ & 5.6 & 563 \\
\hline $\mathcal{Q}_{\text {uotient }_{\sigma}}(W)$ & 2.6 & 549 \\
\hline $\mathcal{S}$ trong $(\sigma)$ & 2.3 & 548 \\
\hline $\mathcal{T}$ ree $(\sigma)$ & 2.7 & 549 \\
\hline
\end{tabular}


ABDERrahim BoussaÏrI

Faculté des Sciences Aïn Chock

DÉpartement de Mathématiques et Informatique

KM 8 ROUTE D'EL JADIDA

BP 5366 MaARIF, Casablanca

MAROC

E-mail address: aboussairi@hotmail.com

Pierre Ille

CNRS, I2M, UMR 7373

13453 Marseille

FRANCE

E-mail address: pierre.ille@univ-amu.fr

RoBert E. WOODRow

Department of Mathematics and Statistics

The University of Calgary

2500 University Drive, Calgary, Alberta

Canada T2N 1N4

E-mail address: woodrow@ucalgary.ca

Received 18 March 2014 